Aus dem Institut für Ethik und Geschichte der Medizin

(Prof. Dr. med. C. Wiesemann)

der Medizinischen Fakultät der Universität Göttingen

\title{
Der Konflikt zwischen \\ Patientenverfügung und \\ Organspendeausweis
}

\author{
Einstellung von ärztlichen und pflegenden Mitarbeitenden \\ der Universitätsmedizin Göttingen
}

\author{
INAUGURAL-DISSERTATION \\ zur Erlangung des Doktorgrades \\ der Medizinischen Fakultät der \\ Georg-August-Universität zu Göttingen
}

vorgelegt von

Marie Luise Lange

aus

Münster

Göttingen 2019 
Dekan:

Referent/in

Ko-Referent/in:

Drittreferent/in:
Prof. Dr. rer. nat. H.K. Kroemer

Prof. Dr. phil. Alfred Simon

Prof. Dr. Jan Liman

Prof. Dr. Margarete Schön

Datum der mündlichen Prüfung: 01.07.2020 
Hiermit erkläre ich, die Dissertation mit dem Titel „Der Konflikt zwischen Patientenverfügung und Organspendeausweis - Einstellung von ärztlichen und pflegenden Mitarbeitenden der Universitätsmedizin Göttingen“ eigenständig angefertigt und keine anderen als die von mir angegebenen Quellen und Hilfsmittel verwendet zu haben.

Göttingen, den

(Unterschrift) 


\section{Inhaltsverzeichnis}

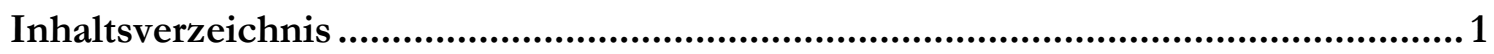

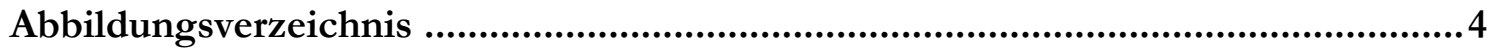

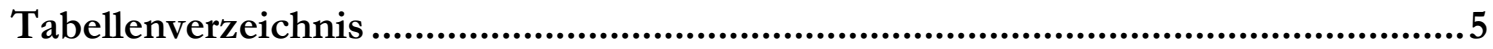

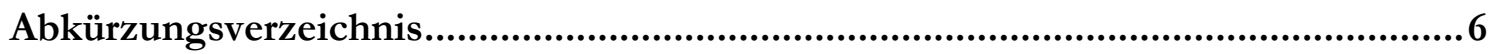

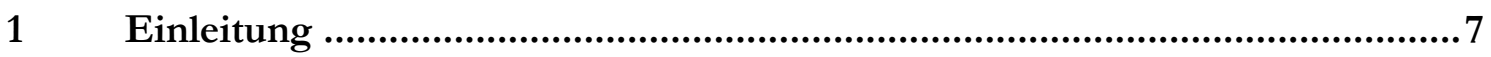

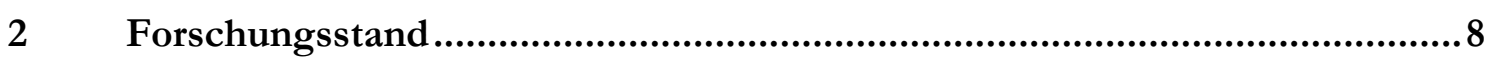

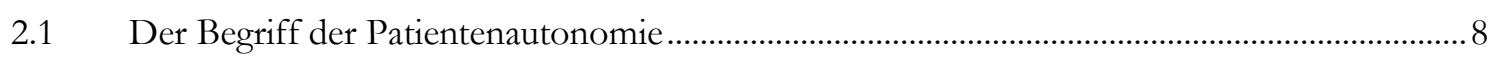

2.1.1 Entwicklung des Begriffs der Patientenautonomie................................................................... 9

2.1.2 Rechtliche Verankerung der Patientenautonomie.................................................................

2.1.3 Zusammenfassung zum Begriff der Patientenautonomie............................................................11

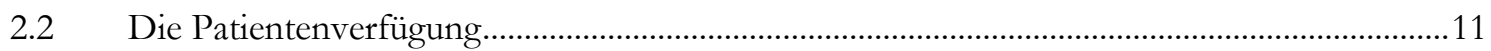

2.2.1 Möglichkeiten der Vorsorge ………………..............................................................11

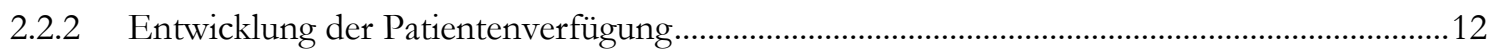

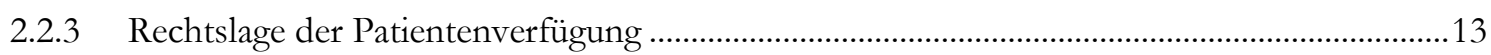

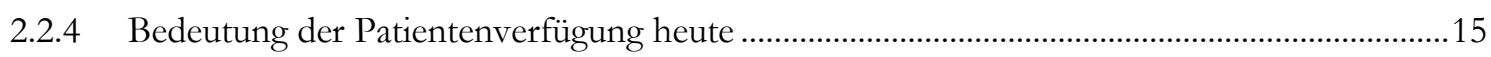

2.2.5 Zusammenfassung zur Patientenverfügung ...........................................................................

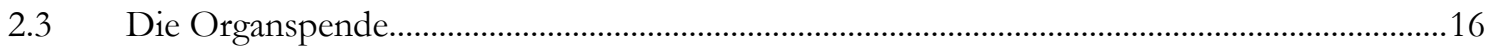

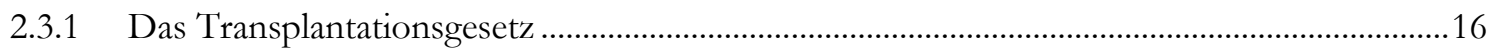

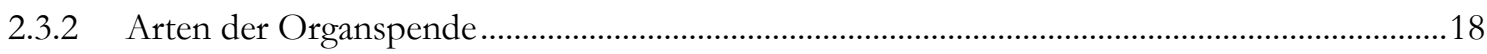

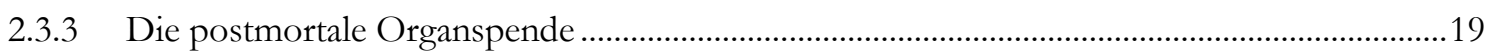

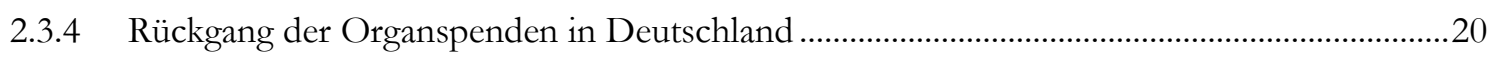

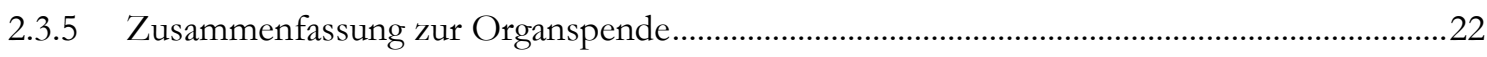

2.4 Der Konflikt zwischen der Patientenverfügung und der Bereitschaft zur Organspende......22

2.4.1 Das Arbeitspapier der B ӒК.............................................................................................2

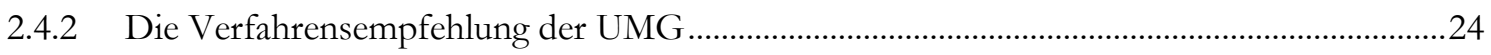

2.4.3 Zusammenfassung zum Konflikt zwischen der Patientenverfügung und der Bereitschaft zur Organspende ................................................................................................26

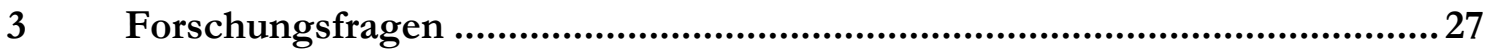

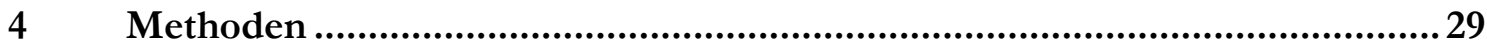

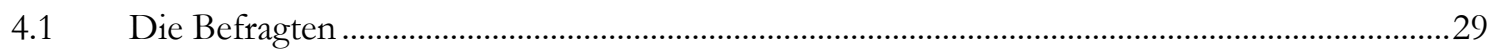

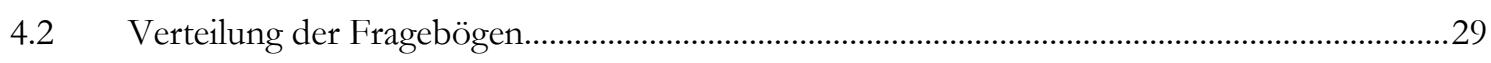

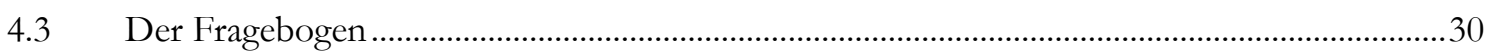


4.3.1 Allgemeine Fragen zur postmortalen Organspende, Organtransplantation und Hirntoddiagnostik

4.3.2 Fragen zur allgemeinen Einstellung gegenüber Organtransplantation,

Hirntoddiagnostik und Patientenverfügung....

4.3.3 Fragen zum Verhältnis von Patientenverfügung und Organspendeerklärung........................31

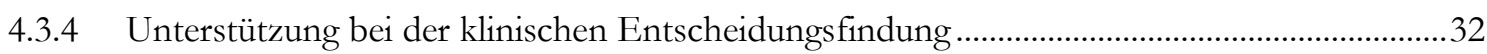

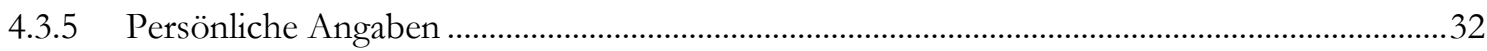

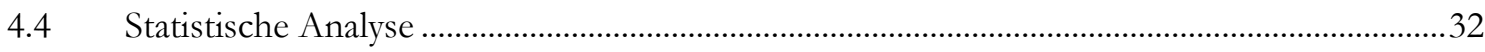

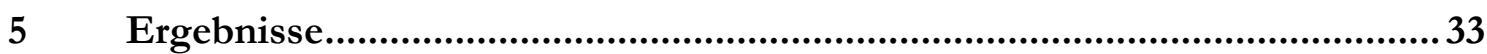

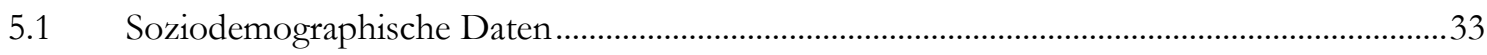

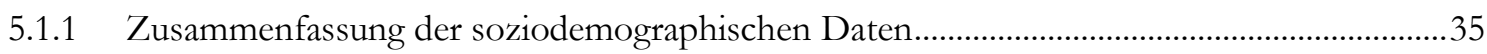

5.2 Einschätzungen und Erfahrungen zur Organspende ...............................................................36

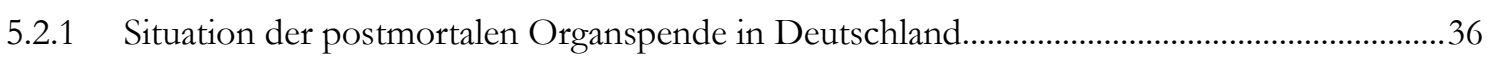

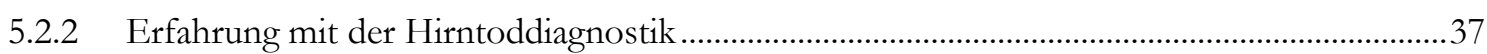

5.2.3 Zusammenfassung zum Thema Einschätzung und Erfahrung zur Organspende .................37

5.3 Fragen zur allgemeinen Einstellung gegenüber Organtrans-plantation,

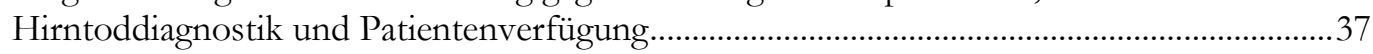

5.3.1 Einstellung zur Organtransplantation und Hirntoddiagnostik ................................................3

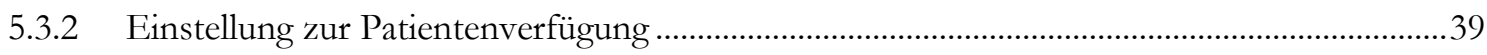

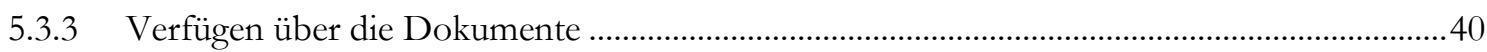

5.3.4 Zusammenfassung zur allgemeinen Einstellung gegenüber Organtransplantation,

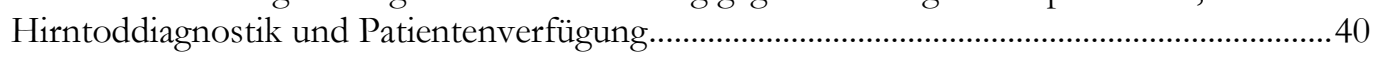

5.4 Verhältnis von Patientenverfügung und Organspendeerklärung ..........................................4 41

5.4.1 Vorgehen bei vermutetem Hirntod: Szenario 1 .....................................................................42

5.4.2 Vorgehen bei Vermutung eines baldig eintretenden Hirntods: Szenario 2.............................45

5.4.3 Zusammenfassung zum Verhältnis von Patientenverfügung und Organspendeerklärung.

5.4.4 Korrelation zwischen grundsätzlichen Einstellungen der Befragten zu ihren

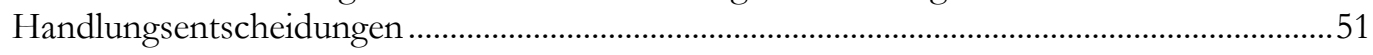

5.5 Einfluss der soziodemographischen Daten auf das Antwort-verhalten................................61

5.6 Unterstützung bei der klinischen Entscheidungsfindung ...................................................61

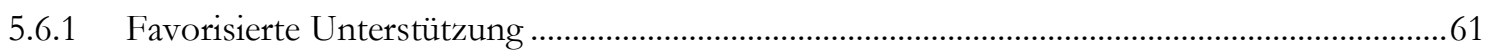

5.6.2 Verfahrensempfehlung der UMG als Unterstützung ........................................................62

5.6.3 Zusammenfassung zur Unterstützung bei der klinischen Entscheidungs-findung ...............63

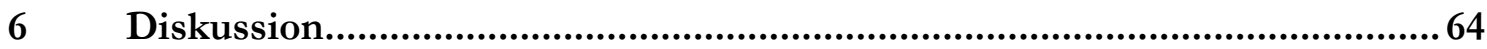

6.1 Bewertung des Konflikts zwischen Patientenverfügung und Organspendeerklärung ..........64

6.1.1 Antwortverhalten der Professionen hinsichtlich der unterschiedlichen Szenarien ...............64

6.1.2 Allgemeine Einstellung zu Organspende, Hirntod und Patientenverfügung ..........................66

6.1.3 Bewertung der Korrelation zwischen den Szenarien.............................................................70

6.1.4 Zusammenfassung des Antwortverhaltens im Zusammenhang mit den Einstellungen

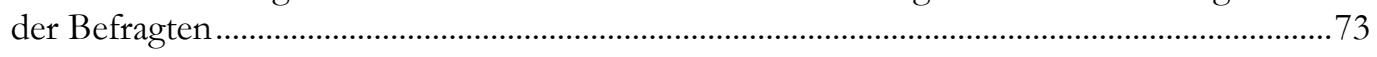

6.2 Unterstützung in schwierigen Entscheidungssituationen ..................................................74

6.2.1 Bewertung der Verfahrensempfehlung der UMG ..................................................................75

6.2.2 Diskrepanz zwischen den Empfehlungen der BÄK und der UMG und der klinischen Realität....... 
6.2.3 Weitere erwünschte Unterstützung ..................................................................................78

6.2.4 Zusammenfassung zur Unterstützung in schwierigen Entscheidungs-situationen ................80

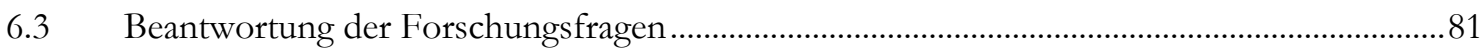

6.3.1 Wie ist die allgemeine Einstellung der ärztlichen und pflegenden Mitarbeitenden der Intensivstationen zu den Themen Organtransplantation, Hirntoddiagnostik und Patientenverfügung?.

6.3.2 Wie sehen die Mitarbeitenden den möglichen Konflikt zwischen Patientenverfügung und Organspendeerklärung?.

6.3.3 Welcher Willenserklärung (Wunsch nach Therapiebegrenzung oder Bereitschaft zur Organspende) würden die Mitarbeitenden im Falle des vermuteten Hirntods den Vorrang geben? Welcher im Falle eines erwarteten Hirntodes? .

6.3.4 Macht es für die Mitarbeitenden bei der Bewertung des möglichen Konflikts einen Unterschied, ob der Wille des Patienten in einer Patientenverfügung bzw.

Organspendeerklärung schriftlich dokumentiert oder stellvertretend von den Angehörigen vorgetragen wid?

6.3.5 Hat die Einstellung der Befragten zu den Themen Organspende und

Patientenverfügung einen Einfluss auf das Handeln bei den Szenarien?

6.3.6 Gibt es Differenzen zwischen den ärztlichen und pflegenden Mitarbeitern hinsichtlich der Einstellung zu den Themen Organspende und Patientenverfügung oder zu dem Vorgehen bei den Szenarien?

6.3.7 Sind das Arbeitspapier der BÄK und die Verfahrensempfehlung der UMG den Mitarbeitenden bekannt? Und wenn ja, als wie hilfreich werden diese eingestuft?

6.3.8 Wünschen sich die Mitarbeitenden Unterstützung bei Entscheidungskonflikten im Zusammenhang mit Patientenverfügung und Organspendeerklärung? Wenn ja, in welcher Form?.

6.3.9 Welchen Einfluss haben Faktoren wie Alter, Geschlecht, Religionszugehörigkeit, berufliche Funktion oder Fachbereich (Klinikzugehörigkeit) auf das Antwortverhalten? ....85

$7 \quad$ Zusammenfassung und Ausblick

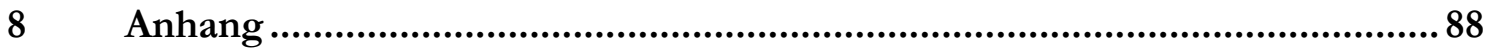

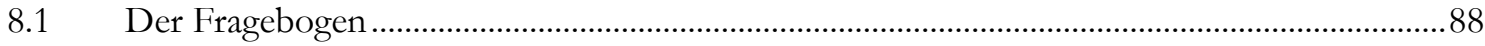

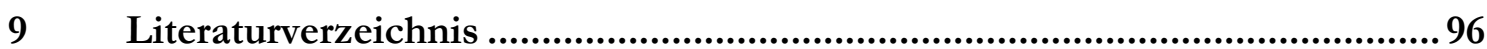




\section{Abbildungsverzeichnis}

Abbildung 1: Gründe für den Rückgang der postmortalen Organspende in Deutschland

(Mehrfachnennung möglich)

Abbildung 2: Zustimmungen beim Thema Organspende und Hirntoddiagnostik. ...............................39

Abbildung 3: Zustimmungen zur Einstellung zum Thema Patientenverfügung..................................40

Abbildung 4: Es wird vermutet, dass der Hirntod beim Patienten bereits eingetreten ist. Eine Organspendebereitschaft ist dokumentiert (Szenario 1a).

Abbildung 5: Es wird vermutet, dass der Hirntod beim Patienten bereits eingetreten ist. Eine Organspendebereitschaft ist unbekannt (Szenario 1b).

Abbildung 6: Es wird vermutet, dass der Hirntod innerhalb der nächsten Tage eintreten wird.

Eine Organspendebereitschaft ist bekannt (Szenario 2a)..........................................................4

Abbildung 7: Es wird vermutet, dass der Hirntod innerhalb der nächsten Tage eintreten wird. Eine Organspendebereitschaft ist unbekannt (Szenario 2b)..................................................4 48

Abbildung 8: Entscheidung für die Maßnahme des Beendens der intensivmedizinischen Maßnahmen bei den unterschiedlichen Szenarien.

Abbildung 9: Betrachtet wurde die Aussage, dass die Organtransplantation eine wichtige Hilfe für Schwerkranke darstelle in Verbindung mit Szenario 2a (baldiger Hirntodeintritt / dokumentierte Organspendebereitschaft).

Abbildung 10: Korrelation zwischen der Aussage, dass die Organtransplantation eine Hilfe für Schwerkranke ist und dem gewählten Vorgehen des Beendens der intensivmedizinischen Maßnahmen im Zusammenhang mit allen Szenarien.

Abbildung 11: Betrachtet wurde die Aussage, dass das Verfahren der BÄK zur Hirntoddiagnostik geeignet ist in Verbindung mit Szenario 1a (eingetretener Hirntod/ dokumentierte Organspendebereitschaft).

Abbildung 12: Betrachtet wurde die Aussage, dass das Verfahren der BÄK zur Hirntoddiagnostik geeignet ist in Verbindung mit Szenario 2b (baldiger Hirntodeintritt/ unbekannte Organspendebereitschaft).

Abbildung 13: Betrachtet wurde die Aussage, dass der Hirntod mit dem Tod des Menschen gleichzusetzen sei in Verbindung mit Szenario 1a (eingetretener Hirntod/ dokumentierte Organspendebereitschaft).

Abbildung 14: Betrachtet wurde die Aussage, dass der Hirntod mit dem Tod des Menschen gleichzusetzen sei in Verbindung mit Szenario 2b (baldiger Hirntodeintritt/ unbekannte Organspendebereitschaft)

Abbildung 15: Betrachtet wurde die Aussage, dass der Tod des Menschen erst bei aufhörendem Herzschlag auftrete, in Verbindung mit Szenario 1a (eingetretener Hirntod/ dokumentierte Organspendebereitschaft).

Abbildung 16: Betrachtet wurde die Aussage, dass die Patientenverfügung keine verbindliche Handlungsanweisung sei, in Verbindung mit Szenario 2b (baldiger Hirntodeintritt/ unbekannte Organspendebereitschaft).

Abbildung 17: Gewünschte Unterstützung für die Szenarien bei der klinischen Entscheidungsfindung (Mehrfachantwort möglich).

Abbildung 18: Bekanntheitsgrad der Verfahrensempfehlung der UMG unter den Befragten. 


\section{Tabellenverzeichnis}

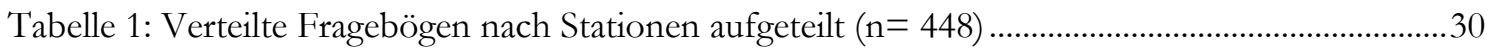

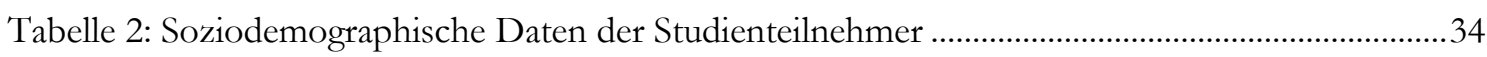

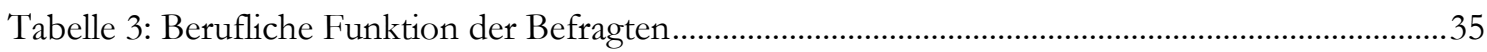

Tabelle 4: Aussagen bei „Sonstiges" des ersten Szenarios .....................................................................45

Tabelle 5: Aussagen bei „Sonstiges" der Frage nach dem ethisch vertretbaren Zeitraum .................49 


\section{Abkürzungsverzeichnis}

$\begin{array}{ll}\text { BÄK } & \text { Bundesärztekammer } \\ \text { BGB } & \text { Bürgerliches Gesetzbuch } \\ \text { BGH } & \text { Bundesgerichtshof } \\ \text { DGHS } & \text { Deutsche Gesellschaft für Humanes Sterben } \\ \text { DSO } & \text { Deutsche Stiftung Organtransplantation } \\ \text { EEG } & \text { Elektroenzephalogramm } \\ \text { GG } & \text { Grundgesetz } \\ \text { TPG } & \text { Transplantationsgesetz } \\ \text { UMG } & \text { Universitätsmedizin Göttingen }\end{array}$




\section{$1 \quad$ Einleitung}

„Organspende und Patientenverfügung - geht das?“ titelte die Deutsche Apothekerzeitung (Deutsche Apotheker Zeitung 2012). Die Frage ist berechtigt: Organe für Transplantationen dürfen erst bei nachgewiesenem Hirntod entnommen werden. Um das Absterben der Organe zu verhindern und ihre Transplantationsfähigkeit zu erhalten, müssen die intensivmedizinischen Maßnahmen bis zur Feststellung des Hirntods und der sich daran anschließenden Organentnahme fortgeführt werden. Doch genau diese Maßnahmen werden in vielen Patientenverfügungen abgelehnt.

Was hat nun Vorrang, die Patientenverfügung oder der Organspendeausweis? Wie sollen ärztliche und pflegende Mitarbeiter mit diesen beiden Willensäußerungen des Patienten verfahren? Der Konflikt gewinnt durch die zunehmende Verbreitung von Patientenverfügungen und Organspendeausweisen im klinischen Alltag immer mehr an Bedeutung. Die vorliegende Studie stellt ein Novum in der Debatte dar, da durch sie erstmals die Einschätzung der ärztlichen und pflegenden Mitarbeiter zu dem Thema erhoben und in den Blick genommen wird. Die vorliegende Studie widmet sich dem Thema, wie die Mitarbeiter der Universitätsmedizin Göttingen (UMG) einen solchen Konflikt einschätzen und vor allen wie sie in solchen Situationen handeln würden. Welche Einstellung haben die Mitarbeiter zu den Themen Organspende, Hirntoddiagnostik und Patientenverfügung? Kommt es tatsächlich aufgrund von Unsicherheiten der handelnden Personen dazu, dass Organspenden - und damit Organtransplantationen - nicht durchgeführt werden?

Beeinflusst dieser Konflikt die Organspendebereitschaft?

Neben der Bewertung des Konflikts zwischen Patientenverfügung und Organspendeausweis entwickelt die vorliegende Studie auch mögliche Lösungsansätze im Lichte bereits veröffentlichter Empfehlungen seitens der Bundesärztekammer (BÄK) sowie der UMG. Daneben wurden den befragten Ärzten und Pflegekräften weitere Vorschläge zur Unterstützung vorgelegt.

Wesentliches Ziel der Studie ist es, die Debatte durch eine Beleuchtung des Konflikts und mögliche Ansätze zur Unterstützung der handelnden Personen in solchen Situationen zu bereichern. Dazu sollte herausgefunden werden, inwieweit der Konflikt überhaupt eine Rolle spielt, wie er sich auf das Handeln der Mitarbeitenden auswirkt und welche möglichen Vorschläge eine Unterstützung im Konflikt darstellen könnten. 


\section{Forschungsstand}

Die Selbstbestimmung des Patienten bei ärztlicher Behandlung gewann in den letzten Jahren immer mehr an Bedeutung, was beispielsweise an geänderten Gesetzen und unterschiedlichsten Richtlinien abzulesen ist. Das bedeutet, ein Patient soll grundsätzlich die Möglichkeit haben, nach seinen Wertevorstellungen autonom zu entscheiden, wie er behandelt werden möchte. Das gilt insbesondere auch für Situationen, in denen er sich selbst nicht mehr äußern kann (BÄK 2013b). Für diese Situationen hat sich das Instrument der Patientenverfügung durchgesetzt. In diesem Dokument kann ein Mensch seine Behandlungswünsche für den Fall fehlender Einwilligungsfähigkeit im Voraus dokumentieren (\$ 1901a BGB). Eine zweite Vorausverfügung neben der Patientenverfügung stellt der Organspendeausweis dar. In diesem kann ein Mensch festlegen, ob er bereit ist, nach seinem Tod Organe und Gewebe zur Transplantation zu spenden ( $\int 2$ Abs. 2 - Gesetz über die Spende, Entnahme und Übertragung von Organen und Geweben - TPG).

Für die behandelnden Ärztinnen und Ärzte sollen solche Vorausverfügungen eine wichtige Hilfestellung sein. Ein Problem kann jedoch entstehen, wenn die Aussagen in diesen Verfügungen einander widersprechen. Dies ist z. B. der Fall, wenn in einer Patientenverfügung lebenserhaltende Maßnahmen abgelehnt werden und gleichzeitig in einem Organspendeausweis die Bereitschaft zur Organspende dokumentiert worden ist. Wie sollen die Ärzte in einer solchen Situation entscheiden und welche Hilfestellungen für solche Entscheidungssituationen gibt es?

Um diese Fragen zu beantworten, wird dem folgenden Kapitel zunächst eine Erläuterung zum Verständnis des Begriffs Autonomie vorangestellt, der grundsätzlich das gegenwärtige Verständnis der Selbstbestimmung des Patienten bestimmt. Im Weiteren werden die beiden davon entscheidend geprägten Dokumente, die Patientenverfügung und der Organspendeausweis in ihrer jeweils möglichen Gestaltung beschrieben. Zur Lösung für eventuelle Konfliktsituationen zwischen den beiden Willensbekundungen eines Patienten wurden von der BÄK und der UMG Hilfestellungen veröffentlicht. Deren Inhalte werden beschrieben und kritisch erläutert.

\subsection{Der Begriff der Patientenautonomie}

Laut Duden entspricht der Begriff der Autonomie dem der Willensfreiheit. Demnach hat ein willensfreier Mensch die Fähigkeit, sich frei und nach seinem eigenem Willen zu ent- 
scheiden (Duden.de - Autonomie: Rechtschreibung, Bedeutung, Definition, Synonyme, Herkunft). Grundlage für solche willensfreie, autonome Entscheidungen sind Informationen sowie eine Auswahl an Alternativen. Darüber hinaus muss der Patient bei seiner Entscheidung frei sein, er darf also nicht fremdbestimmt sein oder Druck verspüren (Bobbert 2002).

\subsubsection{Entwicklung des Begriffs der Patientenautonomie}

Schon zur Zeit der europäischen Aufklärung (1720-1785) bildete sich das neue Selbstbewusstsein des Menschen, gegründet auf seiner Rationalität, heraus (Geisler 2004). Einen wichtigen Beitrag zum heutigen Verständnis von Autonomie lieferte der Philosoph Immanuel Kant mit seiner Formulierung, dass Unmündigkeit das Unvermögen sei, sich seines Verstandes ohne Leitung eines anderen zu bedienen (Kant 1996). Für Kant, dem es auch um die moralische Qualität einer Handlung ging, ist die Vernunft autonom, nämlich selbstgesetzgebend (Störig 1990). So gibt es ein Moralprinzip, auf dessen Grundlage sich alle Handlungen beurteilen lassen. Diesen kategorischen Imperativ „[...] handle nur nach derjenigen Maxime, durch die du zugleich wollen kannst, daß sie ein allgemeines Gesetz werde" formuliert er in anderer Fassung in die Menschheitszweckformel um:

„Nun sage ich: Der Mensch und überhaupt jedes vernünftige Wesen existiert als Zweck ansich selbst, nicht bloß als Mittel zum beliebigen Gebrauche für diesen oder jenen Willen, sondern muß in allen seinen sowohl auf sich selbst als auch auf andere ver nünftige Wesen gerichteten Handlungen jederzeit zugleich als Zweck betrachtet werden."

Demzufolge darf ein Mensch nicht wie eine Sache behandelt werden. Denn Gegenstände haben nur einen relativen Wert, weil sie Mittel zur Verwirklichung der von Menschen subjektiv gewählten eigenen Ziele sind. Wenn aber die Würde eines Menschen zu respektieren ist, dann achtet der Handelnde in der Person die Menschheit - den Menschen als ein Wesen, das sich selbst bestimmen kann. „Handle so, daß du die Menschheit sowobl in deiner Person als in der Person eines jeden andern jederzeit zugleich als Zweck, niemals bloß als Mittel brauchst"'s. Damit dürfen Menschen nie nur als Mittel für etwas missbraucht werden, sie haben nämlich einen absoluten Wert, eine Würde. Diese Würde des Menschen blieb in der medizinischen Entwicklung jedoch nicht immer unangetastet. Der Mensch wurde mehrfach in der Geschichte als Mittel zum Zweck degradiert, insbesondere in der medizinischen Forschung während der Zeit des Nationalsozialismus in Deutschland.

\footnotetext{
${ }^{1}$ Kant, I., Grundlegung zur Metaphysik der Sitten, S.42

$2 \mathrm{ibid}, \mathrm{S} .50$

3 ibid, S.52
} 


\subsubsection{Das Prinzip des ,informed consent"}

Das von den Richtern der Nürnberger Ärzteprozesse postulierte Prinzip des „informed consent" wurde in der zweiten Hälfte des 20. Jahrhunderts auch von der sich immer mehr etablierenden Medizinethik aufgegriffen und weiter entwickelt (Reiter-Theil 2005). Als Hilfestellung für die Behandlung moralischer Probleme und als Grundlage der medizinischen Forschung wurden von Beauchamp und Childress im Jahre 1979 vier Prinzipien formuliert (Beauchamp und Childress 2001). Die Universalisierbarkeit der Prinzipien bei Kant, die allein aus der Vernunft resultieren (Kant 1965), sind nach Beauchamp und Childress nicht ausreichend (Beauchamp und Childress 2001). Bei der ethischen Urteilsbildung sind nämlich neben der Rationalität auch Gefühle, Traditionen und Intuitionen von Bedeutung (Beauchamp und Childress 2001). Dementsprechend beinhalten die Vier Prinzipien von Beauchamp und Childress das Prinzip des Nicht-Schadens, das der Fürsorge, das der Gerechtigkeit und das des Respekts der Autonomie.

Grundlage des „informed consent“ ist der Respekt vor der Autonomie. In der ethischen Debatte werden verschiedene Kriterien für autonome Entscheidungen genannt. Demnach wurden von Beauchamp und Childress zwei Voraussetzungen festgelegt: die Kompetenz und Freiwilligkeit des Patienten. Um dementsprechend das Verständnis des Patienten zu gewährleisten, sollen die Informationen über die Behandlungssituation, verschiedene Behandlungsoptionen sowie mögliche Folgen, Chancen und Risiken offengelegt und eine Empfehlung seitens der Ärztinnen und Ärzte ausgesprochen werden. Auf Grundlage dessen soll der Patient ohne Druck selbst eine Entscheidung fällen und seine Ermächtigung in medizinische Eingriffe erteilen (Beauchamp und Childress 2001). Als Voraussetzungen für autonome Entscheidungen nennen Faden und Beauchamp Verständnis, Absicht und Freiheit (Faden und Beauchamp 1986).

\subsubsection{Rechtliche Verankerung der Patientenautonomie}

Rechtlich folgt das Erfordernis der informierten Einwilligung aus dem Recht des Patienten auf Selbstbestimmung. Dieses folgt aus den im Grundgesetz verankerten Prinzipien bzw. Grundrechten der Menschenwürde (Art. 1 Abs. 1 GG), dem Recht auf Entfaltung der eigenen Persönlichkeit (Art. 2 Abs. 1 i.V.m. Art. 2 Abs. 2 GG) sowie dem Recht auf körperliche Unversehrtheit (Art. 2 Abs. 2 GG). Dies wurde auch von der Rechtsprechung hervorgehoben (BGH, Beschluss vom 17.09.2014 Az. XII ZB 202/13). Darüber hinaus gibt es seit 2013 das Gesetz zur Verbesserung der Rechte von Patientinnen und Patienten, dass u.a. die Rechte des Patienten zusammenfasst. Die Pflichten der Behandelnden, wie eine umfassende und frühzeitige Informations- und Aufklärungspflicht, sowie Regelungen zur 
Dokumentation der Behandlungen wurden festgesetzt. Die Stärkung der Rechte der Patientinnen und Patienten gegenüber Leistungsträgern und bei Behandlungsfehlern bildete einen weiteren Schwerpunkt des Gesetzes. Daneben sollten durch das Gesetz medizinische Handlungsabläufe optimiert und so der Schutz des Patienten gewährleistet werden (bundesgesundheitsministerium.de - Patientenrechte 2016).

\subsubsection{Zusammenfassung zum Begriff der Patientenautonomie}

Der Begriff der personalen Autonomie entwickelte sich schon zu Zeiten der Aufklärung (1720 - 1785). Er wurde in seiner Entstehung geprägt von dem Philosophen Immanuel Kant und im Kontext der Medizin u. a. von den Medizinethikern Beauchamp, Faden und Childress weiterentwickelt. Demnach sind autonome Entscheidungen solche, die von informierten und dadurch kompetenten Patienten sowie aus freien Stücken, also ohne Druck von außen, getroffen werden können. Auch juristisch ist die Patientenautonomie bzw. die Selbstbestimmung des Patienten verankert: u. a. im Grundgesetz sowie im Patientenrechtegesetz, das die Rechte der Patienten gegenüber den medizinischen Leistungserbringern stärken soll.

\subsection{Die Patientenverfügung}

Das Recht des Patienten auf Selbstbestimmung besteht auch über den Zeitpunkt des Verlusts seiner aktuellen Fähigkeit zur Selbstbestimmung hinaus. Dies setzt in der Praxis aber voraus, dass die Behandlungswünsche des Patienten den Behandelnden bekannt sind.

\subsubsection{Möglichkeiten der Vorsorge}

In Deutschland haben Menschen die Möglichkeit, schon im Voraus über ärztliche Maßnahmen für den Fall zu entscheiden, dass sie ihren eigenen Willen nicht mehr äußern können. Dafür gibt es drei unterschiedliche Instrumente: die Patientenverfügung, die Vorsorgevollmacht und die Betreuungsverfügung.

In einer Patientenverfügung kann der (künftige) Patient schriftlich festhalten, wie er in bestimmten Situationen behandelt werden möchte ( $₫$ 1901a Abs. 1-6 BGB). Der Patient kann darin festlegen, welche medizinischen Maßnahmen durchzuführen oder zu unterlassen sind, wenn er sich in der aktuellen Situation nicht mehr äußern kann (Verrel 1999). In der Regel stehen eher negative Behandlungswünsche und Begrenzungen medizinischer Maßnahmen im Fokus der Verfügung. Adressat der Patientenverfügung sind neben dem Patien- 
tenvertreter (Betreuer oder Bevollmächtigter) die behandelnden Ärztinnen und Ärzte sowie das Behandlungsteam.

Mittels einer Vorsorgevollmacht kann eine Vertrauensperson als Stellvertreter benannt werden (Verrel und Simon 2010). Diese ist dann (ebenso wie ein vom Gericht bestellter Betreuer), im Falle der Einwilligungsunfähigkeit des Patienten berechtigt, dessen Willen gegenüber den Ärzten zu vertreten und im Sinne des Patienten in medizinische Maßnahmen einzuwilligen oder diese abzulehnen ( $(1901$ a Abs. 2 BGB). Wenn kein Bevollmächtigter benannt wurde, muss im Falle der Einwilligungsunfähigkeit des Patienten vom Betreuungsgericht ein Betreuer bestellt werden, bei dessen Auswahl verwandtschaftliche sowie persönliche Bindungen vom Gericht berücksichtigt werden (\1896 Abs.1 BGB / \1897 Abs. 5 BGB).

Die dritte Form der Vorsorgemöglichkeiten stellt die Betreuungsverfügung dar. Sie gibt dem Patienten die Möglichkeit, Wünsche hinsichtlich der Person des bestellten Betreuers sowie inhaltliche Wünsche zu Art und Umfang der Betreuung zu äußern.

$\mathrm{Da}$ in dieser Arbeit ein besonderer Fokus auf der Patientenverfügung liegt, wird diese in den nachfolgenden Abschnitten genauer beleuchtet.

\subsubsection{Entwicklung der Patientenverfügung}

In Deutschland etablierten sich der Gedanke sowie die ersten Grundzüge einer Patientenverfügung im Jahre 1978 nach dem Vorbild des amerikanischen „living will" (Dehmel 2006).

Der Jurist Wilhelm Uhlenbruck beschäftigte sich mit medizinethischen Entscheidungen vor allem am Lebensende und forderte schon 1974 das Recht auf einen menschenwürdigen Tod. Vier Jahre später mündeten diese Denkansätze in den sogenannten „Patientenbrief“, der als erstes Patientenverfügungsformular in Deutschland angesehen werden kann (Uhlenbruck 1978). Nach der Veröffentlichung des Patientenbriefs wurden von verschiedenen Organisationen vergleichbare Formulare mit unterschiedlichen Bezeichnungen verfasst. Zunächst gab es den Begriff des „Patiententestaments“. Da der Ausdruck „Testament” jedoch eher mit dem Tod assoziiert ist und eine Patientenverfügung die Behandlung des lebenden Patienten bestimmen soll, wurde der Begriff „Patiententestament“ durch den Begriff „Patientenverfügung“ ersetzt (Geißendörfer 2009). Uhlenbruck erkannte schon damals eventuelle Schwierigkeiten, wie etwa die Interpretationsbreite einer solchen Willensverfügung und die Notwendigkeit eines Bevollmächtigten, der für den äußerungsunfähigen Patienten eintreten soll (Dehmel 2006). 
Die Ärzteschaft stand dem Instrument der Patientenverfügung bis in die 1990er Jahre eher kritisch gegenüber. Die Patientenverfügung wurde als juristisches Dokument betrachtet, das dem Arzt wenig Hilfestellung bei seinen Entscheidungen gibt. Grund für die Skepsis war die Ansicht vieler Ärztinnen und Ärzte, dass der gesunde Patient mögliche problematische Situationen im Krankheitsfall nicht vorhersehen und daher seine Entscheidungen vorwegnehmen könne (BÄK 1993). Durch fortschreitende Überlegungen zu Formulierungen in der Patientenverfügung, angeregt vom jeweiligen Diskussionsstand, konnten im Laufe der Jahre Zweifel ausgeräumt werden, so dass die Skepsis nicht mehr anhielt. Dementsprechend wurde in den Grundsätzen der BÄK zur ärztlichen Sterbebegleitung von 1998 die Patientenverfügung (ebenso wie die Vorsorgevollmacht) als wesentliche Unterstützung für das Handeln des Arztes angesehen. Ferner wurde die Verbindlichkeit der Patientenverfügung betont, sofern sie konkrete Aussagen zur aktuellen Behandlungssituation enthält (BÄK 1998).

Auch rechtlich gewann die Patientenverfügung immer mehr an Bedeutung. Eine entscheidende Bedeutung kam dabei dem „Kemptener Urteil" des Bundesgerichtshofs (BGH) aus dem Jahre 1994 zu (BGH Urteil vom 13.09.1994 Az. 1 StR 357/94). Der BGH stellte damals fest, dass ein Behandlungsabbruch bei einer nicht einwilligungsfähigen Patientin auch dann rechtens ist, wenn der Sterbeprozess noch nicht eingesetzt hat - vorausgesetzt der Behandlungsabbruch entspricht dem mutmaßlichen Willen der Patientin. Dieser müsse aus den Gesamtumständen ermittelt werden, eine besondere Rolle spielten dabei frühere mündliche oder schriftliche Äußerungen der Patientin (Höfling und Schäfer 2006). 2003 präzisierte der BGH seine Aussagen dann dahingehend, dass der in einer Patientenverfügung dokumentierte Wille des Patienten bindend sei (BGH Urteil vom 17.03.2003 Az. XII ZB 2/03). In der Praxis gab es jedoch Verunsicherung im Umgang mit Patientenverfügungen. Diese äußerte sich vor allem im Hinblick auf die Verbindlichkeit in unterschiedlichen Stadien einer Erkrankung sowie zur Rolle des Patientenvertreters (Betreuer oder Bevollmächtigter) bei besonders weitreichenden Entscheidungen. Um mehr Rechtssicherheit zu schaffen, wurde deshalb vom Bundestag 2009 ein Gesetz zur Patientenverfügung beschlossen, das \1901a und \1901b in das BGB einfügte und am 1. September 2009 in Kraft trat (Palandt 2018).

\subsubsection{Rechtslage der Patientenverfügung}

Bei der Patientenverfügung handelt es sich um ein schriftliches Dokument, welches von einem einwilligungsfähigen, volljährigen Menschen freiwillig verfasst wird ( $($ 1901a Abs. 1 BGB). Ein Patient ist dann einwilligungsfähig, wenn er im Stande ist „Art, 
Bedeutung, Tragweite und die Risiken der Maßnahme zu erfassen und seinen Willen biernach zu richten (Palandt 2018). Dabei kommt es nicht im eigentlichen Sinne auf die Geschäftsfähigkeit des Patienten an, sondern auf seine Fähigkeit, die Komplexität und mögliche Folgen des Eingriffs konkret zu erfassen. Voraussetzung für die Einwilligungsfähigkeit sind dementsprechend: Verständnis über die Behandlung, Bewertung mithilfe von Alternativen und Verarbeitung der Informationen (Verrel und Simon 2010).

Unter diesen Voraussetzungen muss vom Patienten konkret festgelegt werden, welche ärztlichen Maßnahmen und Behandlungen erwünscht sind und welche nicht. Wenn der Patientenwille mit der konkreten Lebens- und Behandlungssituation übereinstimmt, dann gilt die Patientenverfügung für das Behandlungsteam als verbindlich - und zwar unabhängig von Art und Stadium der Erkrankung ( $($ 1901a Abs. 1, Abs. 3 BGB). Eine Missachtung der Patientenverfügung und des damit verbundenen Patientenwillens kann für das ärztliche Behandlungsteam sogar als Körperverletzung strafbar sein (Reus 2010).

Wie soll denn nun eine solche Willensäußerung des Patienten aussehen? In Deutschland existiert keine bindende Musterform einer Patientenverfügung, und es gibt keine verbindlichen Richtlinien zur Gestaltung einer Patientenverfügung. Wie bereits erwähnt, ist gesetzlich lediglich festgelegt, dass eine Patientenverfügung in schriftlicher Form vorliegen muss und eigenhändig unterschrieben sein muss. Diese schriftliche Willensäußerung kann jederzeit formlos widerrufen werden, es ist also niemand unwiderruflich an seine Patientenverfügung gebunden (\$ 1901a Abs. 1 Satz 3 BGB). Was aber passiert, wenn keine schriftliche Willenserklärung vorliegt? In diesem Fall muss sich die Behandlung an sonstigen (z.B. mündlichen) Behandlungswünschen bzw. dem mutmaßlichen Willen des Patienten orientieren. Der mutmaßliche Wille ist vom Patientenvertreter (Betreuer oder Bevollmächtigter) des Patienten anhand konkreter Anhaltspunkte (z.B. allgemeine Aussagen oder persönliche Wertvorstellungen des Patienten) zu ermitteln (\$ 1901a Abs. 2 Satz 3 BGB). Unabhängig davon, ob der Wille schriftlich oder mündlich festgehalten wurde, wird vom Patientenvertreter (Betreuer oder Bevollmächtigter) geprüft, ob die festgelegten Wünsche zu den gegenwärtigen Umständen des Patienten passen ( $(1901$ a Abs. 1 BGB). Sobald das nicht zutreffend ist, wird die rechtliche Bindung der Patientenverfügung aufgehoben, sodass wiederum der Betreuer oder Bevollmächtigte (Patientenvertreter) nach dem mutmaßlichen Willen des Patienten Entscheidungen treffen soll ( $\mathbb{1}$ 1901a Abs. 2 BGB).

Der behandelnde Arzt prüft, welche ärztlichen Maßnahmen im Hinblick auf den Gesamtzustand und die Prognose des Patienten indiziert sind ( $\$$ 1901b Abs. 1 BGB). Bei der Fest- 
stellung des Patientenwillen nach \1901a Abs. 1 BGB oder der Behandlungswünsche oder des mutmaßlichen Willens nach \1901a Abs. 2 BGB soll nahen Angehörigen und sonstigen Vertrauenspersonen des Patienten Gelegenheit zur Äußerung gegeben werden, sofern dies ohne erhebliche Verzögerung möglich ist ( $(1901 b$ Abs. 2 BGB).

Eine Genehmigung des Betreuungsgerichts bei Entscheidungen über einen möglichen Behandlungsabbruch nur dann erforderlich, wenn Arzt und Patientenvertreter (Betreuer oder Bevollmächtigter) den (mutmaßlichen) Willen des Patienten unterschiedlich einschätzen und keine Einigung erzielen ( $(1904$ Abs. 1-5 BGB).

\subsubsection{Bedeutung der Patientenverfügung heute}

Die Bekanntheit und Bedeutung der Patientenverfügung hat in den letzten Jahren merklich zugenommen. Vor allem in der älteren Generation ist das Instrument der Vorsorge verbreitet.

So besaßen im Jahr 2003 nur 10 \% der Bundesbürger eine Patientenverfügung. Knapp die Hälfte der Bevölkerung (47\%) wusste seinerzeit gar nicht, was eine Patientenverfügung ist. Auffällig war dabei, dass vor allem die jüngere Generation (unter 30-Jährige) besonders schlecht über das Instrument der Vorsorge aufgeklärt war: nur jeder Vierte kannte den Begriff der Patientenverfügung (DGHS.de - Fakten zur Patientenverfügung 2003). Dahingegen war die Verfügung bei 69 \% der ab 60-Jährigen Bürgern bekannt. Dementsprechend war auch knapp ein Viertel $(23 \%)$ derer, die eine Verfügung hatten, über 60 Jahre alt (DGHS.de - Fakten zur Patientenverfügung 2003).

Seit der gesetzlichen Regelung des Dokuments sechs Jahre später stieg die Zahl der Patientenverfügungen zusehends. Im Jahre 2009 waren es noch $15 \%$, die über eine Patientenverfügung verfügten (IfD-Allensbach.de - Deutlicher Anstieg bei Patientenverfügungen 2014). Fünf Jahre später (2014) hatten fast doppelt so viele der Bürger (28 \%) eine Patientenverfügung, von denen jeder Zweite (51\%) über 60 Jahre alt war (IfD-Allensbach.de - Deutlicher Anstieg bei Patientenverfügungen 2014).

Insgesamt stellt die Einführung der Patientenverfügung eine Erfolgsgeschichte dar: Immer mehr Menschen befassen sich mit der Frage, ob sie lebensverlängernde Maßnahmen in bestimmten Situationen befürworten oder ablehnen. 


\subsubsection{Zusammenfassung zur Patientenverfügung}

Die Patientenverfügung bietet dem (künftigen) Patienten die Möglichkeit, eigene Behandlungswünsche für den Fall des Verlusts der Einwilligungsfähigkeit zu dokumentieren. Ihre Verbindlichkeit ist seit 2009 im Betreuungsrecht geregelt: Demnach muss die Verfügung von einem einwilligungsfähigen Erwachsenen erstellt worden sein und konkrete Aussagen zur aktuellen Behandlungssituation enthalten. Ergänzend zur Patientenverfügung kann der Patient in einer Vorsorgevollmacht eine Vertrauensperson als Bevollmächtigten in Gesundheitsangelegenheiten benennen oder in einer Betreuungsverfügung Vorschläge zur Person des Betreuers sowie zu Art und Umfang der Betreuung machen. Aufgabe des Bevollmächtigten bzw. des Betreuers ist es, auf der Grundlage der Patientenverfügung, sonstiger (z.B. mündlicher) Behandlungswünsche sowie konkreter Hinweise auf den mutmaßlichen Willen den Willen des Patienten bezogen auf die aktuelle Behandlungssituation festzustellen und gegenüber den Ärzten zu vertreten.

\subsection{Die Organspende}

Während die Zahlen der Patientenverfügungen stetig zunehmen (Kapitel 2.2.4) ist seitens der Transplantationsmedizin ein Rückgang an Organspenden zu beobachten (Kapitel 2.3.3). Um diesen Rückgang bewerten zu können, wird im Folgenden auf die Entwicklung und die rechtliche Grundlage der Organspende eingegangen.

\subsubsection{Das Transplantationsgesetz}

Seit 1997 werden die Organspenden in Deutschland durch das Gesetz über die Spende, Entnahme und Übertragung von Organen und Gewebe - dem Transplantationsgesetz (TPG) geregelt. Das Gesetz stellt Voraussetzungen für die unterschiedlichen Arten der Organspende auf und beschäftigt sich außerdem mit der Organisation und Vermittlung von Organspenden an Bedürftige. Ferner werden Einrichtungen benannt, die Transplantationen durchführen dürfen. Organisatorisch und personell werden dabei die Bereiche Organspende, Organübertragung und Organvermittlung streng voneinander getrennt. Ziel des Gesetzes ist es, die Bereitschaft zur Organspende in Deutschland zu fördern ( 1 Abs. 1 Satz 1 TPG).

Das Gesetz gilt für die Spende und die Entnahme menschlicher Organe, Organteile oder Gewebe zur Übertragung auf andere Menschen ( $\mathbb{1} 1$ Abs. 2 Satz 1 TPG). Dabei unterscheidet das Gesetz die Organentnahme bei toten und bei lebenden Spendern. Voraussetzung für die postmortale Organspende ist die Zustimmung des Verstorbenen zu Lebzeiten sowie 
die vorherige Feststellung des Hirntodes durch zwei entsprechend qualifizierte Ärzte. Hat der Verstorbene seinen Willen bezogen auf die Organspende zu Lebzeiten nicht dokumentiert, muss sein nächster Angehöriger über die Organspende entscheiden. Dieser hat bei seiner Entscheidung den mutmaßlichen Willen des Verstorbenen zu berücksichtigen. Bei der Lebendorganspende muss der Spender volljährig sein und in die Organspende nach entsprechender Aufklärung eingewilligt haben. Seine Gesundheit darf durch den Eingriff nicht über das normale OP-Risiko hinausgehend gefährdet sein. Außerdem muss eine unabhängige Kommission bestätigen, dass die Spende freiwillig erfolgt und ein Organhandel ausgeschlossen werden kann.

Ebenfalls Inhalt des Gesetzes sind Auflagen für Transplantations- oder Entnahmezentren. Außerdem werden die Vermittlung und auch die Organisation der Transplantation als Schwerpunkte des Gesetzes behandelt. Die Übertragung und die Entnahme der Organe darf demnach nur in zugelassenen Zentren vorgenommen werden ( $\int 9 \mathrm{a}$ TPG). Von den zugelassenen Transplantationszentren müssen Listen über Patienten geführt werden, die ein Organ benötigen. Die Reihenfolge ergibt sich aus Kriterien wie Dringlichkeit und Erfolgschancen der Transplantation. Koordiniert und organisiert werden die Transplantationen durch die Deutsche Stiftung für Organtransplantation (DSO) (DSO.de - Jahresbericht 2015: Organspende und Transplantation in Deutschland 2016). Koordinatoren der Stiftung veranlassen die nötigen Untersuchungen und bereiten die Operationen vor. Daneben gewährleistet die Stiftung, dass der Wille des Spenders vertreten wird. Das gilt ebenso für die Interessen des Empfängers. Auch dient die Stiftung als Ansprechpartner für die Angehörigen. Für die Vermittlung der Organe ist eine andere Stiftung zuständig: die gemeinnützige Stiftung Eurotransplant. Sobald dort eine Information über ein Spenderorgan eingeht, ermittelt Eurotransplant nach den gesetzlichen Richtlinien einen geeigneten Empfänger (Heberer et al. 2013). Eurotransplant vermittelt zwischen den Ländern Belgien, Deutschland, Kroatien, Luxemburg, den Niederlanden, Österreich, Ungarn und Slowenien (DSO.de Jahresbericht 2015: Organspende und Transplantation in Deutschland 2016).

Warum werden die Bereiche der Koordination und Vermittlung von zwei unterschiedlichen Stiftungen betreut? Diese Trennung der Aufgabenbereiche ist bewusst gewählt, da so der Handel mit Organen ausgeschlossen werden soll. Nach dem Transplantationsgesetz ist Organhandel verboten und wird strafrechtlich verfolgt (\$17 Abs. 1 TPG / $\ 18$ TPG).

In dieser Form bestand das Transplantationsgesetz rund fünfzehn Jahre, bis Reformen zur Organspende erlassen wurden. Durch die „Entscheidungslösung“ aus dem Jahre 2012 sollte eine umfassende Aufklärung der Bevölkerung gesichert sowie die Möglichkeit einer of- 
fenen Kommunikation mit Angehörigen und deren gesetzlichen Vertretern geschaffen werden (Heberer et al. 2013). Demnach soll jede Person ab dem 16. Lebensjahr in zweijährigem Abstand von den Krankenkassen mit Informationsmaterial und Organspendeausweisen ausgestattet werden (bundesgesundheitsministerium.de - Gesetz zur Neuregelung der Organspende tritt in Kraft 2012). Die „Entscheidungslösung", welche die Bitte nach einer Stellungnahme zur Organspende erweitert und konkretisiert hat, löste damit die bisherige „Zustimmungslösung" ab (Heberer et al. 2013). Nicht nur die Information über das Thema Organspende in der Bevölkerung sollte verbessert werden, sondern auch die Koordination der Organspenden. Dafür muss jede Organentnahmeklinik nun auch über einen Transplantationsbeauftragten verfügen ( $\sqrt{9 \mathrm{~b}} \mathrm{Abs} .1 \mathrm{TPG}$ ). Abläufe und Strukturen der Spenden sollen durch den Beauftragten verbessert werden (Schäfer 2012).

\subsubsection{Arten der Organspende}

Grundsätzlich kann zwischen zwei Arten der Organspende unterschieden werden: die Lebendspende und die postmortale Organspende. Die ersten Versuche einer Organtransplantation waren Lebendspenden. Eine Nierentransplantation wurde bereits im Jahre 1936 von dem ukrainischen Chirurgen Yurii Voronoy durchgeführt. Allerdings war das Organ nicht funktionstüchtig und die Patientin verstarb wenige Tage nach der Operation. Rund zwanzig Jahre später im Jahre 1954 wurde in Boston dann die erste erfolgreiche Nierentransplantation zwischen eineiigen Zwillingen durchgeführt (Ethikrat.org - Hirntod und Entscheidung zur Organspende: Stellungnahme 2015).

Worin unterscheiden sich die beiden Formen der Organspende? Bei einer Lebendspende werden Organe, Gewebe und Zellen von einem lebenden Menschen auf einen anderen übertragen. Dabei wird ein Organ von paarweise vorhandenen Organen gespendet oder Teile von Organen, Geweben oder Zellen mit einer hohe Regenerationsfähigkeit, sodass für den Spender keine lebensbedrohlichen Einschränkungen entstehen (Pilz 2007). Neben der Lebendspende gibt es noch die Möglichkeit einer postmortalen Organspende. Voraussetzung für die postmortale Organspende ist der sog. dissoziierte Hirntod, d.h. der irreversible Ausfall der Gesamtfunktion des Großhirns, des Kleinhirns und des Hirnstamms bei maschinell aufrechterhaltenem Kreislauf. Das bedeutet, dass Organe und Gewebe im Unterschied zur Lebendspende erst nach dem Tod des Spenders gespendet werden. Ferner muss eine Zustimmung zur Organ- und Gewebespende durch den Spender selbst oder durch die Angehörigen erfolgen (\$ 3 Abs. 1 TPG / \$ 4 TPG). 


\subsubsection{Die postmortale Organspende}

Da sich die vorliegende Arbeit vor allem mit der postmortalen Organspende beschäftigt, wird diese im Folgenden genauer thematisiert. Besonderer Wert wird dabei auf das Hirntodkriterium gelegt. Wie bereits erläutert ist der festgestellte Tod des Patienten die Grundlage für eine postmortale Organspende. Doch wie ist der Tod des Menschen zu definieren und was bedeutet der Hirntod?

Das Gesetz besagt, dass der Tod des Spenders nach dem Stand der Erkenntnisse der medizinischen Wissenschaft festgestellt werden muss ( $\int 3$ Abs. 1 Satz 2 TPG). Die entsprechenden Verfahrensregeln dazu werden von der BÄK festgelegt.

\subsubsection{Hirntodkriterien der BÄK}

Die BÄK hat gemäß dem $\int 16$ TPG Richtlinien zur Diagnostik des Spendertodes aufgestellt: Verfahrensregeln zur Feststellung des irreversiblen Hirnfunktionsausfalls. Dieser Hirnfunktionsausfall wird beschrieben als nicht behebbarer Ausfall der Gesamtfunktion des Großhirns, des Kleinhirns und des Hirnstamms. Drei Kriterien müssen erfüllt sein, um den irreversiblen Hirnfunktionsausfall bestätigen zu können: eine Hirnschädigung muss vorliegen, klinische Symptome müssen festgestellt werden, und ein Irreversibilitätsnachweis muss gemacht werden (aerzteblatt.de - Richtlinie der BÄK 2015).

Demzufolge muss dem Hirnfunktionsausfall eine akute schwere primäre oder sekundäre Hirnschädigung voraus gehen. Primäre Hirnschädigungen wie Hirnverletzungen, intrakranielle Blutungen und Hirninfarkte betreffen das Gehirn direkt und strukturell. Sekundäre Hirnschädigungen schädigen das Gehirn indirekt, beispielsweise infolge einer Hypoxie oder eines kardial bedingten Kreislaufstillstandes. Sämtliche anderen Ursachen müssen ausgeschlossen werden. Aus der Hirnschädigung resultiert eine unwiederbringlich erloschene Gesamtfunktion von Großhirn, Kleinhirn und Hirnstamm. In diesem Zustand ist der Organismus nicht mehr fähig, selbstständig lebenswichtige Funktionen aufrechtzuerhalten. Der Ausfall der Hirnfunktionen spiegelt sich in folgenden klinischen Symptomen wider: Bewusstlosigkeit, Hirnstammareflexie und Atemstillstand. Die Symptome müssen eine definierte Zeit über angedauert haben, damit das Kriterium der Irreversibilität gewährleistet ist. Der Zeitrahmen ist auf eine Dauer von mindestens zwölf Stunden bei primärer und auf mindestens drei Tage bei sekundärer Hirnschädigung festgelegt. Ergänzende Untersuchungen wie ein Null-Linien-EEG, das Erlöschen evozierter Potentiale oder der zerebrale Zirkulationsstillstand können ebenfalls zum Irreversibilitätsnachweis genutzt werden (aerzteblatt.de - Richtlinie der BÄK 2015). 
Die genannten Kriterien müssen von zwei Ärzten unabhängig voneinander festgestellt werden. Weder in die Organübertragung noch in die Entnahme dürfen die Ärztinnen und Ärzte, die für die Hirntoddiagnostik verantwortlich sind, involviert sein. Die gewissenhafte Durchführung der Hirntoddiagnostik wird anhand standardisierter Protokolle sichergestellt (Losch und Hoffner 2015). Während der Durchführung der Hirntoddiagnostik müssen die intensivmedizinischen Maßnahmen fortgeführt werden, um die Versorgung der möglicherweise zu transplantierenden Organe zu gewährleisten (BÄK 2013a).

\subsubsection{Rückgang der Organspenden in Deutschland}

In Deutschland herrscht ein deutlicher Mangel an Organspenden (Breyer et al. 2007): Es sterben täglich drei Personen in Deutschland an akutem Organversagen (Klinkhammer und Hibbeler 2007). Am 31. Dezember 2016 standen rund 10.128 Menschen in Deutschland auf der Warteliste für ein Spenderorgan. Nur etwa ein Drittel, 3.708 Organe, wurden 2016 bundesweit übertragen, allerdings muss dabei zwischen postmortaler Spende, Lebendspende und sogenannter Dominospende differenziert werden. Die transplantierten Organe nach postmortaler Spende nahmen mit 82,2\% (3.049) der insgesamt 3.708 gespendeten Organe den Großteil ein, 647 (17,4\%) waren Lebendspenden und 12 (0,3 \%) Dominospenden. Unter einer Dominotransplantation versteht man beim Empfänger die Entnahme des kranken Organs, wobei von diesem kranken Organ dann mögliche noch vitale und gesunde Teile an einen weiteren Empfänger gespendet werden können. Da sich die vorliegende Studie mit der postmortalen Organspende beschäftigt und diese auch den größten Teil der transplantierenden Organe einnimmt, wird im Folgenden ein besonderer Fokus auf die postmortale Organspende gelegt. Geht man zurück und vergleicht die Zahlen der postmortalen Organspende mit denen aus dem Jahre 2011, wird der Rückgang der Spender sehr deutlich: 2011 gab es 3.917 postmortale Organspenden - also rund 1.000 postmortale Organspenden mehr als im Jahre 2016 (2.867). Zu beachten ist, dass dabei nur die tatsächlich transplantierten Organe zu den gespendeten Organen zählen (DSO.de - Jahresbericht 2016: Organspende und Transplantation in Deutschland 2017).

\subsubsection{Gründe für den Rückgang der Organspenden in Deutschland}

Ist dieser Rückgang durch eine kritischere Einstellung der Bevölkerung gegenüber einer Organspende zu erklären? Eine generelle Ablehnung der Organspende ist jedenfalls bei Betrachtung der Zahlen der DSO nicht zu beobachten. Bezogen auf die postmortalen Spenden ist 2016 bei 1.248 Patienten der Hirntod nach dem von der BÄK vorgegebenen Verfahren festgestellt worden. Sie galten dementsprechend als mögliche Organspender. Bei 
$68 \%$ (857) dieser Patienten wurde tatsächlich eine Organspende durchgeführt. Bei den anderen $32 \%$, wo also keine Organtransplantation realisiert werden konnte, spielte neben einem geringen Anteil von medizinischen Kontraindikationen (6 \%) als Hauptgrund (24\%) eine fehlende Zustimmung zur Organspende eine Rolle. Es ist also nicht von einer generellen Ablehnung gegenüber der Organspende zu sprechen, aber immerhin bei fast jedem 4. eine kritische Haltung zur Organspende zu beobachten (DSO.de - Jahresbericht 2016: Organspende und Transplantation in Deutschland 2017).

Ein grundsätzliches Einverständnis der Bevölkerung wurde auch bei einer im Jahr 2014 durchgeführten Umfrage der Bundeszentrale für gesundheitliche Aufklärung (BZgA) deutlich: Es waren über zwei Drittel (71 \%) der Befragten grundsätzlich mit einer Organspende einverstanden, jedoch verfügten nur $35 \%$ der Teilnehmenden über einen Organspendeausweis (DSO.de - Repräsentativbefragung: Einstellung, Wissen und Verhalten der deutschen Allgemeinbevölkerung zur Organ- und Gewebespende 2014). Die Diskrepanz zwischen der Bereitschaft zur Organspende und dem Vorliegen eines Organspendeausweises führt zu einer Nichtausschöpfung der Spendebereitschaft im Fall des Todes und ist als einer der Gründe für den Organmangel in Deutschland anzusehen (Ethikrat.org - Die Zahl der Organspenden erhöhen 2007). Vor diesem Hintergrund wurde in Deutschland 2012 die oben erwähnte Regelung eingeführt, dass die Krankenkassen ihre Versicherten regelmäßig über die Organspende informieren müssen.

Der beschriebene Rückgang der Spenden ist mit hoher Wahrscheinlichkeit auch auf die Skandale im Zusammenhang mit der Meldung und Allokation von Organen an der Uniklinik in Göttingen sowie an anderen Kliniken zurückzuführen (aerzteblatt.de - Zahl der Organspenden weiter rückläufig 2017). Wobei zu beachten ist, dass die Zahlen auch schon vor den Skandalen rückläufig waren (DSO.de - Jahresbericht 2015: Organspende und Transplantation in Deutschland 2016). Die DSO sieht deshalb als maßgeblichen Grund für den Rückgang der Organspendebereitschaft das fehlende Bewusstsein zur Spende in den Krankenhäusern an (aerzteblatt.de - Zahl der Organspenden weiter rückläufig 2017). Auch die zunehmende Zahl der Patientenverfügungen könnte ein weiterer Auslöser für den Rückgang der Organspenden sein. Wenn gemäß der Patientenverfügung lebenserhaltende Maßnahmen beendet werden, bevor der Hirntod eingetreten bzw. festgestellt worden ist, dann kommt auch eine Organspende nicht mehr zustande, selbst wenn der Patient sein Bereitschaft zur Organspende in einem Organspendeausweis bekundet hat. Hier ergibt sich ein Konflikt zwischen den beiden Instrumenten der Willensbekundungen des Patienten (aerztezeitung.de - Weniger Organspenden: Das Problem Patientenverfügung 2012). 


\subsubsection{Zusammenfassung zur Organspende}

Trotz vieler Erneuerungen und Aktualisierungen des seit 1997 bestehenden Transplantationsgesetzes sind die Zahlen der Organspende auf einem niedrigen Niveau und sinken stetig. Unabhängig davon, ob es sich dabei um eine Lebendspende handelt, bei der von einem lebenden Patienten ein Organ oder ein Teil eines Organs übertragen wird oder ob es sich um eine postmortale Organspende handelt. Bei der postmortalen Organspende muss der sog. Hirntod vorliegen, der durch bestimmte, festgelegte Verfahrensregeln der BÄK zu diagnostizieren ist.

Als Gründe für den Rückgang der Organspende ist allerdings nicht eine generelle kritische Einstellung gegenüber der Spende von Organen in der Bevölkerung maßgeblich, sondern ein Zusammenspiel von vielen Faktoren: Die Transplantationsskandale, eine mangelnde Berücksichtigung der Organspende in den Krankenhäusern und auch der Konflikt zwischen mehreren Willensäußerungen des Patienten, z. B. der Patientenverfügung und dem Organspendeausweis (siehe Kapitel 2.4).

\subsection{Der Konflikt zwischen der Patientenverfügung und der Bereitschaft zur Organspende}

Die gesetzlichen Regelungen des Patientenverfügungsgesetzes im Jahre 2009 bewirkten eine Zunahme der Anzahl an Patientenverfügungen sowie eine stärkere Berücksichtigung antizipierter Willensäußerungen, wie beispielsweise des Organspendeausweises (BÄK 2013a). Allerdings können die Patientenverfügung und der Organspendeausweis einander widersprechende Anweisungen enthalten. Wie bereits dargestellt, setzt die Realisierung einer Organspende voraus, dass intensivmedizinische Maßnahmen bis zum Eintritt bzw. der Feststellung des Hirntodes und der Entnahme der Organe weitergeführt werden. Darüber hinaus sind ggf. zusätzliche organprotektive Maßnahmen erforderlich. Diese Maßnahmen werden nicht zur Verbesserung des Zustandes des Patienten, sondern mit Blick auf die vom Patienten gewünschte Organspende durchgeführt. Deshalb besteht die Möglichkeit, dass die intensivmedizinischen Maßnahmen der Patientenverfügung widersprechen. Für die Ärzte stellt sich dann die Frage: Welcher der beiden Willenserklärungen, der im Organspendeausweis dokumentierten Bereitschaft zur Organspende oder dem in der Patientenverfügung festgelegten Wunsch nach Therapiebegrenzung, soll in der konkreten Situation der Vorrang eingeräumt werden?

Als Hilfestellung für die behandelnden Ärztinnen und Ärzte hat die BÄK 2013 ein Arbeitspapier zum Verhältnis von Patientenverfügung und Organspendeerklärung veröffent- 
licht. Basierend auf diesem Arbeitspapier hat die UMG im Jahr 2014 eine Verfahrensempfehlung für den Umgang mit Patientenverfügung und Organspendeerklärung im intensivmedizinischen Alltag erarbeitet (med.uni-goettingen.de - Wunsch nach Therapiebegrenzung und Bereitschaft zur Organspende 2014). Beide Dokumente haben einen empfehlenden Charakter, sind aber rechtlich nicht bindend (BÄK 2013a).

\subsubsection{Das Arbeitspapier der BÄK}

Das Arbeitspapier zum Verhältnis von Patientenverfügung und Organspendeerklärung wurde im März 2013 im Deutschen Ärzteblatt veröffentlicht. Es beschreibt verschiedene Fallkonstellationen, die sich im Zusammenhang mit dem oben beschriebenen Konflikt ergeben können, und wertet diese rechtlich wie ethisch aus. Darüber hinaus enthält das Arbeitspapier Hilfestellungen zum Umgang mit Konfliktsituationen sowie Textbausteine, mit deren Hilfe Bürgerinnen und Bürger das Verhältnis von Patientenverfügung und Organspende in ihrer Vorausverfügung selbst regeln können (BÄK 2013a).

Den Ausgangspunkt der Überlegungen bildet die Situation, dass ein Patient, der als potentieller Organspender in Frage kommt, beatmet auf einer Intensivstation liegt. Für die erste Gruppe von Fallkonstellationen wird angenommen, dass der Patient eine Patientenverfügung hat, in der er (weitere) intensivmedizinische Maßnahmen für die aktuelle Situation ablehnt. Zugleich hat der Patient einen Organspendeausweis, in dem er seine Bereitschaft zur Organspende dokumentiert hat. Für diese Konstellation werden dann folgende Situationen unterschieden und bewertet:

- Situation 1 (,vermuteter Hirntod“): Die behandelnden Ärzte nehmen an, dass der Hirntod bereits eingetreten ist.

- Situation 2 (,,erwarteter Hirntod“): Die behandelnden Ärzte vermuten, dass der Hirntod innerhalb weniger Tage eintreten wird.

- Situation: „Reanimation“

In Situation 1 (,vermuteter Hirntod“) besteht nach Einschätzung der BÄK kein Widerspruch zwischen dem Wunsch nach Therapiebegrenzung und der Bereitschaft zur Organspende. Zur Feststellung des Hirntodes und der sich daran ggf. anschließenden Organspende ist eine zeitlich eng befristete Fortführung der intensivmedizinischen Maßnahmen erforderlich. Aufgrund der Organspendeerklärung könne - so die BÄK - davon ausgegangen werden, dass der Patient mit dieser trotz des grundsätzlichen Wunsches nach Therapiebegrenzung einverstanden war. 
Diese Annahme treffe auf die Situation 2 („erwarteter Hirntod“) nicht zu. Aus der Bereitschaft zur Organspende könne nicht automatisch abgeleitet werden, dass der Patient mit der Fortführung der intensivmedizinischen Maßnahmen einverstanden sei. Die Fortführung der intensivmedizinischen Maßnahmen führe nämlich dazu, dass der Sterbeprozess über den Zeitraum hinaus verlängert würde, in dem der Hirntod festgestellt und eine Organentnahme durchgeführt werden könnte. Wann der Hirntod tatsächlich eintrete, sei schwer zu prognostizieren. Daher wisse man auch nicht, wie lange die intensivmedizinischen Maßnahmen fortgeführt werden müssten. Die Empfehlung geht dahin, dass der Arzt zusammen mit einem Patientenvertreter (Betreuer oder Bevollmächtigter) und den Angehörigen des Patienten die Entscheidung treffen solle. Dabei sei auch über die mit der Fortführung der intensivmedizinischen Maßnahmen verbundenen Risiken aufzuklären. Da sowohl eine Organspendeerklärung als auch eine Patientenverfügung vorliegen, müssten beide Erklärungen bei der Feststellung des Patientenwillens berücksichtigt werden.

Im Fall des Herzstillstands und einer erforderlichen Reanimation zum Zweck der Behandlung und Wiederherstellung der Gesundheit des Patienten (Situation Reanimation) empfiehlt die BÄK, dass eine Reanimation rechtlich unzulässig und ethisch nicht vertretbar sei. Eine Reanimation sei ein erheblicher Eingriff, der von der Organspendeerklärung nicht gedeckt sei.

\subsubsection{Die Verfahrensempfehlung der UMG}

Basierend auf dem Arbeitspapier der BÄK hat das Klinische Ethik-Komitee zusammen mit Vertretern der zuständigen Kliniken der UMG eine Verfahrensempfehlung zum Umgang mit Patientenverfügung und Organspendeerklärung im intensivmedizinischen Alltag veröffentlicht. Inhaltlich entsprechen sich die beiden Dokumente, wobei die Verfahrensempfehlung der UMG deutlich kürzer und stärker handlungsorientiert ist. Außerdem sind die Situationen etwas anders strukturiert (med.uni-goettingen.de - Wunsch nach Therapiebegrenzung und Bereitschaft zur Organspende 2014).

In den Verfahrensempfehlungen wird ebenfalls unterschieden zwischen der Situation des vermuteten und der des erwarteten Hirntodes. Bei den Handlungsempfehlungen für diese beiden Situationen wird dann differenziert, ob die Bereitschaft des Patienten zur Organspende bekannt oder nicht bekannt ist.

- Situation 1: Intensivmedizinische Maßnahmen bei vermutetem Hirntod:

Die Bereitschaft des Patienten zur Organspende impliziere, dass der Patient damit einverstanden sei, dass die intensivmedizinischen Maßnahmen zeitlich eng begrenzt durchgeführt 
werden dürften, nämlich bis zur Hirntodfeststellung und einer ggf. sich anschließenden Organentnahme. Intensivmedizinische Maßnahmen seien also nur zu unterlassen, wenn bekannt sei, dass der Patient eine Organspende ablehnt.

- Situation 2: Intensivmedizinische Maßnahmen bei erwartetem Hirntod:

Die Lage stelle sich hier anders da. Aus der Bereitschaft zur Organspende allein könne nicht abgeleitet werden, dass der Patient mit der Fortführung der Maßnahmen auch in dieser Situation einverstanden sei, weil die Fortführung der intensivmedizinischen Maßnahmen den Sterbeprozess nicht nur kurzfristig verlängere, sondern solange es für die Feststellung des Hirntods und die Durchführung der Organspende notwendig ist. Die Schwierigkeit liege darin, dass das Leben bis zum Hirntod verlängert werden müsste, dessen Eintrittszeitpunkt schwer zu prognostizieren sei. Es sei ferner nicht sicher, ob der Hirntod überhaupt eintreten werde.

Wenn die Bereitschaft des Patienten zur Organspende bekannt sei, solle der Arzt gemeinsam mit einem eventuell vorhandenen Patientenvertreter (Betreuer oder Bevollmächtigter) entscheiden, ob die Fortführung der intensivmedizinischen Maßnahmen zur Realisierung der vom Patienten gewünschten Organspende dem mutmaßlichen Willen des Patienten entspreche. Die UMG empfiehlt, eine Frist für die Fortsetzung der intensivmedizinischen Maßnahmen festzulegen und die Maßnahmen zu beenden, wenn in dieser Zeit der Hirntod nicht eingetreten sei.

Intensivmedizinische Maßnahmen seien zu unterlassen, wenn bekannt sei, dass der Patient eine Organspende ablehnt.

Wenn nicht bekannt ist, ob der Patient zur Organspende bereit ist oder nicht, sei gemeinsam mit den nächsten Angehörigen und dem Patientenvertreter (Betreuer oder Bevollmächtigter) zu klären, ob der Patient zu einer Organspende bereit wäre und ob die dafür notwendige befristete Fortführung der intensivmedizinischen Maßnahmen dem mutmaßlichen Willen des Patienten entspricht.

Für den Fall, dass den behandelnden Ärzten eine Ablehnung der Organspende seitens des Patienten bekannt ist, wird eine Fortführung intensivmedizinischer Maßnahmen also zum Zweck der Organspende in jedem Fall als unzulässig betrachtet, unabhängig davon, ob der Hirntod vermutet oder erwartet wird. 


\subsubsection{Zusammenfassung zum Konflikt zwischen der Patientenverfügung und der Bereitschaft zur Organspende}

Sowohl die Patientenverfügung als auch der Organspendeausweis enthalten Willensbekundungen des Patienten. Diese können auf den ersten Blick einander widersprechende Anweisungen enthalten. Das ist beispielsweise der Fall, wenn lebenserhaltende Maßnahmen in einer Patientenverfügung abgelehnt werden und zugleich eine Organspende erwünscht ist, für deren Realisierung allerdings wiederum intensivmedizinische Maßnahmen erfolgen müssten. Um den Umgang mit den beiden Dokumenten für solche Situationen zu erleichtern, gibt es für das behandelnde Personal Hilfestellungen der BÄK und basierend darauf auch eine Empfehlung der UMG, die stärker praxisorientierter ist. Beide Hilfestellungen beinhalten Fallkonstellationen, bei denen zum einen der Hirntod als maßgeblich angesehen wird und zum anderen zwischen dem Zeitpunkt seines Eintritts (vermuteter bzw. erwarteter Hirntod) und sodann je nach dem Vorliegen von Dokumenten (Patientenverfügung und Organspendeausweis) und deren Inhalt variiert wird. Inhaltlich decken sich die beiden Empfehlungen: Vorgeschlagen wird, dass bei einem Patienten, dessen Hirntod bereits vermutlich eingetreten ist (vermuteter Hirntod), trotz des Konflikts der beiden Dokumente eine Organspende realisierbar wäre. Sobald der Hirntod jedoch innerhalb weniger Tage eintreten würde (erwarteter Hirntod), soll mit dem Patientenvertreter (Betreuer oder Bevollmächtigter) und den Angehörigen eine Entscheidung getroffen werden. Die intensivmedizinischen Maßnahmen sind beiden Empfehlungen nach immer unzulässig, wenn der Patient sich gegen eine Organspende geäußert hat. 


\section{Forschungsfragen}

Wie im vorangegangenen Kapitel erläutert wurde, kann es zu einem Konflikt zwischen dem in einer Patientenverfügung festgelegten Wunsch nach Therapiebegrenzung und der in einem Organspendeausweis dokumentierten Bereitschaft zur Organspende kommen. Als Hilfestellung zur Lösung des Konflikts wurden das Arbeitspapier der BÄK und die Verfahrensempfehlung der UMG vorgestellt.

Ziel der vorliegenden Studie ist es, die Einstellung von ärztlichen und pflegenden Mitarbeitenden der Intensivstationen der UMG zum Thema Patientenverfügung und Organspendeerklärung zu erheben. Ferner soll untersucht werden, ob das Arbeitspapier der BÄK und die Verfahrensempfehlung der UMG bekannt sind, ob diese als hilfreich angesehen werden bzw. welche anderen Formen der Unterstützung bei entsprechenden Entscheidungskonflikten von den Mitarbeitenden gewünscht sind.

Konkret sollen folgende Forschungsfragen beantwortet werden:

- Wie ist die allgemeine Einstellung der ärztlichen und pflegenden Mitarbeitenden der Intensivstationen zu den Themen Organtransplantation, Hirntoddiagnostik und Patientenverfügung?

- Wie sehen die Mitarbeitenden den möglichen Konflikt zwischen Patientenverfügung und Organspendeerklärung?

- Welcher Willenserklärung (Wunsch nach Therapiebegrenzung oder Bereitschaft zur Organspende) würden die Mitarbeitenden im Falle des vermuteten Hirntods den Vorrang geben? Welcher im Falle eines erwarteten Hirntodes?

- Macht es für die Mitarbeitenden bei der Bewertung des möglichen Konflikts einen Unterschied, ob der Wille des Patienten in einer Patientenverfügung bzw. Organspendeerklärung schriftlich dokumentiert oder stellvertretend von den Angehörigen vorgetragen wird?

- Hat die Einstellung der Befragten zu den Themen Organspende und Patientenverfügung einen Einfluss auf das Handeln bei den Szenarien?

- Gibt es Differenzen zwischen den ärztlichen und pflegenden Mitarbeitern hinsichtlich der Einstellung zu den Themen Organspende und Patientenverfügung oder zu dem Vorgehen bei den Szenarien? 
- Sind das Arbeitspapier der BÄK und die Verfahrensempfehlung der UMG den Mitarbeitenden bekannt? Und wenn ja, als wie hilfreich werden diese eingestuft?

- Wünschen sich die Mitarbeitenden Unterstützung bei Entscheidungskonflikten im Zusammenhang mit Patientenverfügung und Organspendeerklärung? Wenn ja, in welcher Form?

- Welchen Einfluss haben Faktoren wie Alter, Geschlecht, Religionszugehörigkeit, berufliche Funktion oder Fachbereich (Klinikzugehörigkeit) auf das Antwortverhalten? 


\section{$4 \quad$ Methoden}

Zur Beantwortung der oben genannten Forschungsfragen wurde eine empirische Befragung mittels Fragebogen durchgeführt.

\subsection{Die Befragten}

Da es in der Befragung u. a. um die Verfahrensempfehlung der UMG ging, fand diese unter den Mitarbeitenden der UMG statt. Als Probanden wurden Ärztinnen und Ärzte, Pflegende sowie auch Studierende und Auszubildende ausgewählt, die zum Zeitpunkt der Durchführung der Studie auf einer der Intensivstationen der UMG tätig waren und für die das Thema Patientenverfügung und Organspendeerklärung sich im Arbeitsalltag stellt bzw. jederzeit stellen kann. Konkret wurden die ärztlichen du pflegenden Mitarbeiter der intensivmedizinischen Stationen der Klinik für Anästhesiologie, der Klinik für Nephrologie und Rheumatologie, der Klinik für Kardiologie und Pneumologie, der Klinik für pädiatrische Kardiologie und Intensivmedizin, der Klinik für Gastroenterologie und Endokrinologie, der Klinik für Allgemein-, Viszeral- und Kinderchirurgie, der Klinik für Neurologie und Rheumatologie sowie auch der Klinik für Neurochirurgie befragt.

\subsection{Verteilung der Fragebögen}

Im ersten Schritt wurden die jeweiligen Direktoren der ausgewählten Kliniken per E-Mail über das Vorhaben dieser Umfrage informiert und um ihre Zustimmung gebeten. Bei vorliegender Erlaubnis wurden im Folgenden die Stationsleitungen sowie die Oberärztinnen und Oberärzte der jeweiligen Stationen angeschrieben um herauszufinden, wie viele ärztliche und pflegende Mitarbeitende auf den unterschiedlichen Stationen tätig sind. Anhand dieser Angaben wurden 448 Fragebögen im Februar 2015 anonym auf den Stationen verteilt (Tabelle 1).

Die Teilnahme an dieser Befragung war freiwillig. Den Teilnehmenden wurde ein Monat Zeit gegeben, um den beantworteten Fragebogen mit der Hauspost zurückzuschicken.

Nach Ablauf der Frist wurden im März 2015 die Oberärztinnen und Oberärzte sowie die Stationsleitungen per E-Mail gebeten, die Mitarbeitenden an die Umfrage zu erinnern. Den Teilnehmenden wurden weitere vier Wochen zur Beantwortung des Fragebogens eingeräumt. 
Tabelle 1: Verteilte Fragebögen nach Stationen aufgeteilt $(\mathrm{n}=448)$

\begin{tabular}{|l|c|c|}
\hline \multicolumn{1}{|c|}{ Station } & $\begin{array}{c}\text { Ärztliche Mit- } \\
\text { arbeitende } \\
\mathrm{n}=111\end{array}$ & $\begin{array}{c}\text { Pflegende } \\
\text { Mitarbeitende } \\
\mathrm{n}=337\end{array}$ \\
\hline $\begin{array}{l}\text { Intermediate Care Station (Station 1025) } \\
\text { Kardiologische Intensivstation (Station 1026) }\end{array}$ & 10 & 30 \\
Intermediate Care Station (Station 1021) & 10 & 35 \\
Nephrologische Intensivstation (Station 1022) & 7 & 25 \\
Neurologische Intensivstation (Station 1013) & 10 & 38 \\
Allgemein- und Viszeralchirurgie (Station 1012) & 10 & 30 \\
$\begin{array}{l}\text { Allgemeinchirurgische Intensivstation (Station 1014) } \\
\text { Intermediate Care Station }\end{array}$ & & 30 \\
$\begin{array}{l}\text { Orthopädie / Unfallchirurgie / Neurochirurgie } \\
\text { (Station 3011) }\end{array}$ & 4 & 22 \\
$\begin{array}{l}\text { Anästhesiologie / Neurochirurgie - } \\
\text { Operative Intensivstation (Station 0117) }\end{array}$ & & 30 \\
$\begin{array}{l}\text { Anästhesiologie / Thorax-Herz-Gefäßchirurgie - Opera- } \\
\text { tive Intensivstation (Station 0118) }\end{array}$ & 43 & 20 \\
Interdisziplinäre Pädiatrische und Neonatologische Inten- \\
sivstation (Station 0133)
\end{tabular}

\subsection{Der Fragebogen}

Der Fragebogen „Befragung zum Thema Patientenverfügung und Organspendeerklärung“ gliederte sich in fünf Abschnitte. Im Folgenden werden die Inhalte dieser Abschnitte übersichtsartig dargestellt. Eine Kopie des Fragebogens befindet sich im Anhang.

\subsubsection{Allgemeine Fragen zur postmortalen Organspende, Organtransplantation und Hirntoddiagnostik}

Im ersten Abschnitt ging es um allgemeine Fragen zur postmortalen Organspende, Organtransplantation und Hirntoddiagnostik. Der Rückgang der Organtransplantationen in Deutschland und mögliche Gründe dafür wurden thematisiert. Ferner wurden berufliche Berührungspunkte mit der postmortalen Organspende sowie der Wissensstand über die Durchführung einer Hirntoddiagnostik erfragt. Und schließlich wurde gefragt, ob die Teilnehmenden selbst einen Organspendeausweis und/oder eine Patientenverfügung hatten. 


\subsubsection{Fragen zur allgemeinen Einstellung gegenüber Organtransplantation, Hirntoddiagnostik und Patientenverfügung}

Die allgemeine Einstellung gegenüber Organtransplantation, Hirntoddiagnostik und Patientenverfügung wurde im zweiten Abschnitt des Fragebogens erfragt. Es wurden Aussagen vorgelegt, die von den Teilnehmenden mit „stimme voll und ganz zu“, „stimme eher zu“,

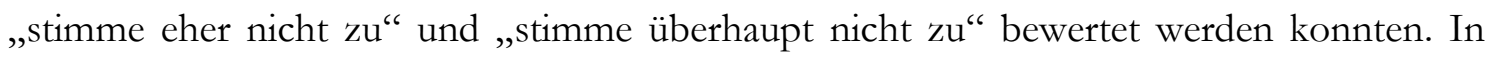
den ersten vier Aussagen ging es um die allgemeine Einstellung zu Organtransplantation, Hirntod und Hirntoddiagnostik, in den folgenden drei um die Einstellung zur bzw. die Einschätzung der Patientenverfügung.

\subsubsection{Fragen zum Verhältnis von Patientenverfügung und Organspendeerklärung}

Der dritte Abschnitt des Fragebogens enthielt Fragen zum Verhältnis von Patientenverfügung und Organspendeerklärung. Dazu wurden den Probanden vier Szenarien präsentiert. Mit Hilfe der Szenarien sollte ein Praxisbezug hergestellt werden, der es den Probanden erleichtern sollte, sich in verschiedene Situationen hineinzuversetzen und sich für bestimmte Handlungen zu entscheiden. Die Szenarien orientierten sich an den im Arbeitspapier der BÄK und in der Verfahrensempfehlung der UMG unterschiedenen Situationen.

Die Ausgangssituation aller vier Szenarien war wie folgt: Ein Patient liegt nach einem schweren Verkehrsunfall mit einer Hirnblutung auf der Intensivstation. Er ist bewusstlos und wird maschinell beatmet. Der Patient hat eine Patientenverfügung, in der er lebenserhaltende Maßnahmen für den Fall einer schweren irreversiblen Hirnschädigung ablehnt.

Diese Ausgangssituation wurde in einem ersten Schritt wie folgt variiert:

Szenario 1: Die behandelnden Ärzte vermuten, dass der Hirntod bereits eingetreten ist.

Szenario 2: Die behandelnden Ärzte vermuten, dass der Hirntod innerhalb der nächsten Tage eintreten wird.

Diese beiden (Grund-)Szenarien wurden dann in einem zweiten Schritt wie folgt unterteilt:

a) Der Patient hat seine Bereitschaft zur Organspende in einem Organspendeausweis dokumentiert (Szenario 1a und 2a).

b) Die Bereitschaft des Patienten zur Organspende ist nicht bekannt (Szenario 1b und $2 b)$. 
Mithilfe vorgegebener Antwortoptionen sowie eines Freitextfeldes („Sonstiges, und zwar:") wurden die Teilnehmenden befragt, für welches Vorgehen sie sich entscheiden würden.

\subsubsection{Unterstützung bei der klinischen Entscheidungsfindung}

Im vierten Abschnitt des Fragebogens wurden die Teilnehmenden befragt, ob sie sich Unterstützung bei entsprechenden Entscheidungskonflikten wünschen, und wenn ja, in welcher Form. In diesem Zusammenhang wurde auch nach dem Arbeitspapier der BÄK und der Verfahrungsanweisung der UMG gefragt.

Wegen eines Formatierungsfehlers war die Frage nach dem Arbeitspapier der BÄK jedoch leider nicht lesbar und konnte dementsprechend nicht mit ausgewertet werden.

\subsubsection{Persönliche Angaben}

Im fünften und letzten Abschnitt wurden persönliche Daten der Teilnehmenden wie Geschlecht, Alter, Religionszugehörigkeit, berufliche Funktion und Klinikzugehörigkeit erfragt.

\subsection{Statistische Analyse}

Die statistische Auswertung der zurückgesendeten Fragebögen erfolgte mittels der Programme Microsoft Excel 2010 und Statistica 13.0.

Eine Sammlung der Daten sowie eine erste Auswertung erfolgte durch Excel. Alle Fragen, bei denen die Möglichkeit bestand, eine Antwort frei zu formulieren, wurden separat ausgewertet.

Zur Auswertung der Szenarien wurden durch das Programm Statistica die Merkmale des Antwortverhaltens der Aussagen und des Vorgehens untersucht und ihre Beziehung zueinander festgestellt. So konnte eine zweidimensionale Häufigkeitsverteilung hergestellt werden. Der Kolmogorow-Smirnow-Test wurde zur Überprüfung der Übereinstimmung zweier Wahrscheinlichkeitsverteilungen angewendet. Die Signifikanzgrenze des p-Werts wurde auf $<0,05$ festgelegt. 


\section{$5 \quad$ Ergebnisse}

Von den insgesamt 433 verteilten Fragebögen wurden 114 ausgefüllt zurückgeschickt. Dies entspricht einer Rücklaufquote von $26 \%$. Unter den 114 Teilnehmenden befanden sich 30 ärztliche (26\%) und 84 pflegende Mitarbeitende (74\%).

\subsection{Soziodemographische Daten}

Die pflegenden Mitarbeitenden waren mehrheitlich weiblich (68\%) und zwischen 40 und 59 Jahre alt (40-49 Jahre: $33 \%$, 50-59 Jahre: $37 \%$ ). Bei den ärztlichen Mitarbeitenden war der Anteil der Männer mit 57 \% etwas höher als der der Frauen (43 \%). Über die Hälfte der Ärztinnen und Ärzte (53 \%) war zwischen 30 und 39 Jahre alt. (Tabelle 2)

Zwei Drittel der ärztlichen (68\%) sowie $59 \%$ der pflegenden Befragten gehörten einer Religionsgemeinschaft an. Von den Teilnehmenden mit Religionszugehörigkeit waren die ärztlichen mehrheitlich katholisch, die pflegenden mehrheitlich evangelisch. Vier ärztliche Teilnehmende gaben an (12\%), einer anderen Religionsgemeinschaft anzugehören: zwei von ihnen bekannten sich zum Buddhismus, die beiden anderen machten keine genauere Angabe. $32 \%$ der ärztlichen und $41 \%$ der pflegenden Mitarbeitenden gehörten keiner Religionsgemeinschaft an. (Tabelle 2)

Ein Drittel der ärztlichen und pflegenden Befragten (37\%) war in der Klinik für Anästhesiologie tätig. Ebenfalls 36 \% der Ärztinnen und Ärzte arbeitete in der pädiatrischen Kardiologie und Intensivmedizin - unter den pflegenden Mitarbeitenden befanden sich nur $10 \%$ in dieser Klinik. Weitere Kliniken, aus denen die Beschäftigten kamen, waren die Klinik für Kardiologie und Pneumologie (12\%), die Klinik für Nephrologie und Rheumatologie (12\%), die Klinik für Neurologie (12\%), die Klinik für Allgemein-, Viszeral- und Kinderchirurgie (10\%) und die Klinik für Gastroenterologie und Endokrinologie (1\%). (Tabelle 2) 
Tabelle 2: Soziodemographische Daten der Studienteilnehmer

\begin{tabular}{|c|c|c|c|}
\hline & $\begin{array}{c}\text { Ärztliche Mitarbei- } \\
\text { tende } \\
\mathrm{n}=30\end{array}$ & $\begin{array}{l}\text { Pflegende Mitarbei- } \\
\text { tende } \\
\text { n }=84\end{array}$ & $\begin{array}{l}\text { Gesamt } \\
\mathrm{n}=114\end{array}$ \\
\hline \multicolumn{4}{|l|}{ Geschlecht } \\
\hline Weiblich & $43 \%$ & $68 \%$ & $61 \%$ \\
\hline Männlich & $57 \%$ & $32 \%$ & $39 \%$ \\
\hline \multicolumn{4}{|l|}{ Alter } \\
\hline$<30$ Jahre & $7 \%$ & $12 \%$ & $10 \%$ \\
\hline 30-39 Jahre & $53 \%$ & $17 \%$ & $26 \%$ \\
\hline 40-49 Jahre & $30 \%$ & $33 \%$ & $32 \%$ \\
\hline 50-59 Jahre & $10 \%$ & $37 \%$ & $31 \%$ \\
\hline$>60$ Jahre & $0 \%$ & $1 \%$ & $1 \%$ \\
\hline \multicolumn{4}{|l|}{ Religionsgemeinschaft } \\
\hline Keine & $32 \%$ & $41 \%$ & $39 \%$ \\
\hline Römisch- katholisch & $32 \%$ & $21 \%$ & $23 \%$ \\
\hline Evangelisch & $24 \%$ & $38 \%$ & $35 \%$ \\
\hline Andere Religion & $12 \%$ & $0 \%$ & $3 \%$ \\
\hline \multicolumn{4}{|l|}{ Klinik } \\
\hline $\begin{array}{l}\text { Klinik für Allgemein-, Viszeral-, } \\
\text { und Kinderchirurgie }\end{array}$ & $4 \%$ & $12 \%$ & $10 \%$ \\
\hline Klinik für Anästhesiologie & $36 \%$ & $37 \%$ & $37 \%$ \\
\hline $\begin{array}{l}\text { Klinik für Gastroenterologie und } \\
\text { Endokrinologie }\end{array}$ & $0 \%$ & $1 \%$ & $1 \%$ \\
\hline $\begin{array}{l}\text { Klinik für Kardiologie und } \\
\text { Pneumologie }\end{array}$ & $10 \%$ & $12 \%$ & $12 \%$ \\
\hline $\begin{array}{l}\text { Klinik für Nephrologie und } \\
\text { Rheumatologie }\end{array}$ & $7 \%$ & $14 \%$ & $12 \%$ \\
\hline Klinik für Neurologie & $7 \%$ & $14 \%$ & $12 \%$ \\
\hline $\begin{array}{l}\text { Klinik für pädiatrische Kardiolo- } \\
\text { gie und Intensivmedizin }\end{array}$ & $36 \%$ & $10 \%$ & $16 \%$ \\
\hline
\end{tabular}


Mehr als die Hälfte (63\%) der teilnehmenden Ärztinnen und Ärzte war als Stationsärztin/ -arzt oder als Assistenzärztin/ -arzt tätig. $17 \%$ des ärztlichen Personals arbeitete als Oberärztin/ -arzt in den Kliniken. Bei den pflegenden Mitarbeitenden waren $5 \%$ in leitender Funktion tätig . $90 \%$ arbeiteten als Gesundheits- und Krankenpflegerin/ -pfleger auf den jeweiligen Stationen. (Tabelle 3)

Tabelle 3: Berufliche Funktion der Befragten

\begin{tabular}{|c|c|c|c|}
\hline & $\begin{array}{c}\text { Ärztliche Mitarbei- } \\
\text { tende } \\
\mathrm{n}=30\end{array}$ & & $\begin{array}{c}\text { Pflegende Mitar- } \\
\text { beitende } \\
\mathrm{n}=84\end{array}$ \\
\hline Leiterin/ Leiter der Klinik & $0 \%$ & Stationsleitung & $5 \%$ \\
\hline $\begin{array}{l}\text { Leitende Oberärztin/ Leitender } \\
\text { Oberarzt }\end{array}$ & $3 \%$ & $\begin{array}{l}\text { Gesundheits- und } \\
\text { Krankenpfleger/ - } \\
\text { pflegerin }\end{array}$ & $90 \%$ \\
\hline Oberärztin/ Oberarzt & $17 \%$ & $\begin{array}{l}\text { Auszubildender/ } \\
\text { Auszubildende }\end{array}$ & $0 \%$ \\
\hline $\begin{array}{l}\text { Stationsärztin/ Stationsarzt / } \\
\text { Assistenzärztin/ Assistenzarzt }\end{array}$ & $63 \%$ & Keine Angabe & $5 \%$ \\
\hline PJ-lerin/ PJ-ler & $7 \%$ & & \\
\hline Studentin/ Student & $3 \%$ & & \\
\hline Keine Angabe & $3 \%$ & & \\
\hline
\end{tabular}

\subsubsection{Zusammenfassung der soziodemographischen Daten}

Die Soziodemographischen Daten unterschieden sich bezüglich der Professionen. Der Großteil der Teilnehmenden waren weibliche Pflegende, die zwischen 40-59 Jahre alt waren und keiner oder der evangelischen Religion angehörten. Die ärztlichen Befragten hingegen waren eher männlich, deutlich jünger (30-39 Jahre) und gehörten keiner oder der katholischen Religion an. Sowohl unter den pflegenden, als auch unter den ärztlichen Beschäftigten nahmen eher weniger in leitenden Positionen an der Befragung teil. Dementsprechend handelte sich bei den Pflegenden überwiegend um Gesundheits- und Krankenpflegerinnen/ -pfleger und bei den ärztlichen Mitarbeitern um Stationsärztinnen/ -ärzte oder als Assistenzärztinnen/ -ärzte. Ebenfalls fiel auf, dass ein Hauptteil der Antwortenden aus beiden Professionen in der Klinik für Anästhesiologie arbeitete, wobei von den Ärztinnen und Ärzten ein genau so großer Anteil in der pädiatrischen Kardiologie und Intensivmedizin tätig war. 


\subsection{Einschätzungen und Erfahrungen zur Organspende}

Im ersten Teil des Fragebogens wurde näher auf die Organspende eingegangen, im speziellen wurden Einschätzungen zur rücklaufenden Zahl der postmortalen Organspenden abgegeben. Anschließend wurde die Erfahrung der Befragten mit der Hirntoddiagnostik begutachtet.

\subsubsection{Situation der postmortalen Organspende in Deutschland}

Mehr als zwei Drittel der befragten ärztlichen und pflegenden Mitarbeitenden (70 bzw. $75 \%$ ) waren der Ansicht, dass in dem Rückgang der Zahl der postmortalen Organspenden in Deutschland ein längerfristiger Trend zum Ausdruck komme. Als Grund hierfür sah ein Drittel der Befragten (31 bzw. $30 \%$ ) die Skandale in der Transplantationsmedizin in den letzten Jahren an. Als weitere mögliche Gründe wurden Zweifel an der Sicherheit der Hirntoddiagnostik (17 bzw. $21 \%$ ), ethische Bedenken gegenüber dem Hirntodkriterium (10 bzw. $16 \%$ ), mangelnde Aufklärung der Öffentlichkeit (15 bzw. $13 \%$ und die gesetzliche Regelung zur Organspende in Deutschland (14 bzw. $9 \%$ ) angegeben. Kein ärztlicher und nur einzelne pflegende Mitarbeitende (0 bzw. $5 \%$ ) sahen die wachsende Zahl an Patientenverfügungen als Grund für den Rückgang der Zahl der postmortalen Organspenden an. Die mangelnde finanzielle Entschädigung der Spenderkrankenhäuser (2 bzw. $1 \%$ ) sowie die mangelnde Kooperation zwischen Spenderkrankenhäusern und Transplantationszentren (5 bzw. $3 \%$ ) wurden ebenfalls nur von wenigen als Grund für den Rückgang der postmortalen Organspenden angegeben. (Abb.1)

Abbildung 1: Gründe für den Rückgang der postmortalen Organspende in Deutschland (Mehrfachnennung möglich).

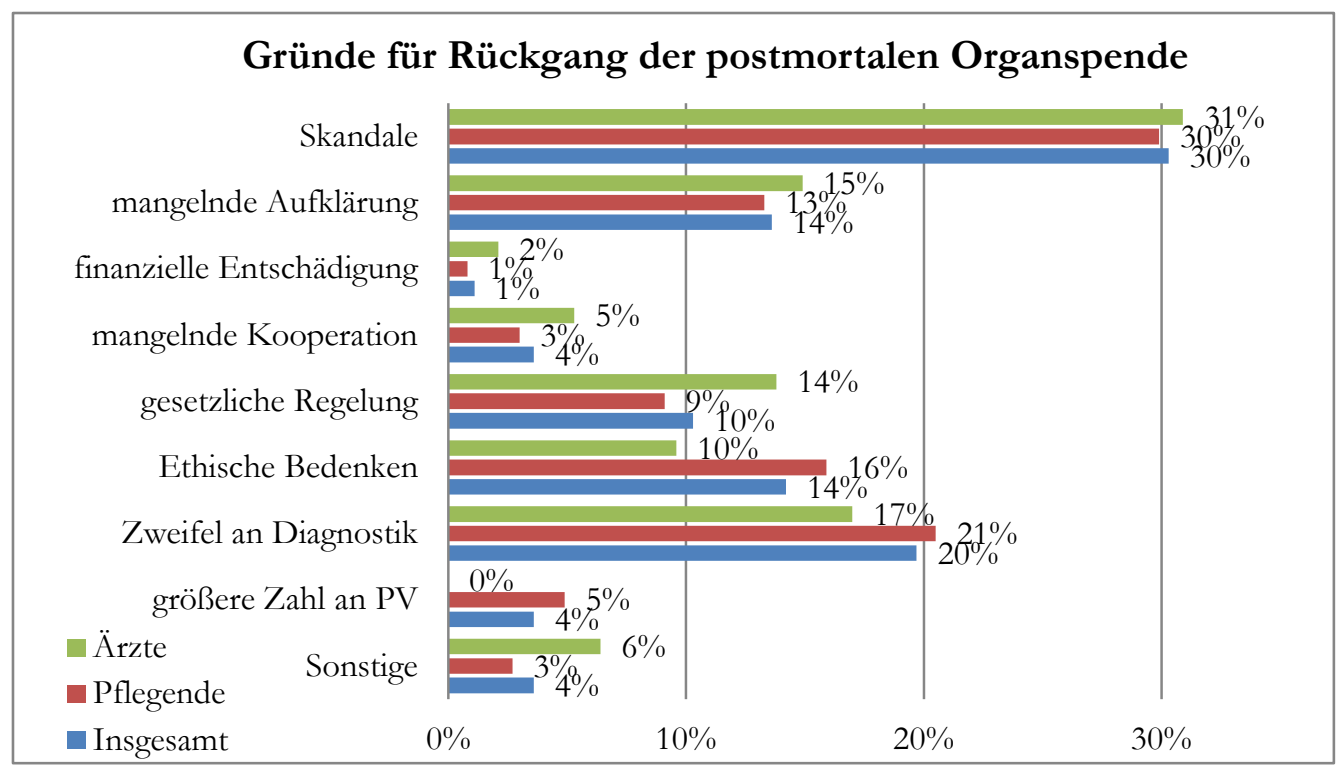




\subsubsection{Erfahrung mit der Hirntoddiagnostik}

Die große Mehrheit der Befragten (90\%) gab an, im Rahmen der bisherigen beruflichen Tätigkeit bereits mit dem Thema der postmortalen Organspende in Berührung gekommen zu sein: das Pflegepersonal am häufigsten im Zusammenhang mit der Betreuung von Organspendern (80\%) sowie im Zusammenhang mit der Hirntoddiagnostik (83\%). Die Ärztinnen und Ärzte am häufigsten im Zusammenhang mit der Betreuung von Organempfängern (77\%). $10 \%$ der Befragten gaben an, keine berufliche Erfahrung mit dem Thema Organspende zu haben (Ärztinnen/Ärzte: $13 \%$; Pflegende: $8 \%$ ).

Etwas mehr als die Hälfte der Befragten (55\%) war nach eigener Einschätzung mit den Kriterien zur Durchführung einer Hirntoddiagnostik vertraut. Bei einer Hirntoddiagnostik selbst anwesend waren mehr pflegende (66\%) als ärztliche Mitarbeiter (53\%).

\subsubsection{Zusammenfassung zum Thema Einschätzung und Erfahrung zur Organspende}

Zusammenfassend sehen die Befragten den Rückgang der postmortalen Organspenden in Deutschland als längerfristigen Trend, der hauptsächlich auf den Skandalen in der Transplantationsmedizin beruhe, wobei auch Skepsis an der Hirntoddiagnostik geäußert wurde. Während der beruflichen Tätigkeit hatte die Mehrheit der Teilnehmenden bereits mit dem Thema der postmortalen Organspende zu tun und war anwesend bei oder vertraut mit der Durchführung einer Hirntoddiagnostik.

\subsection{Fragen zur allgemeinen Einstellung gegenüber Organ- transplantation, Hirntoddiagnostik und Patientenverfügung}

Im zweiten Teil des Fragebogens wurde die allgemeine Einstellung der Teilnehmenden gegenüber Organtransplantation, Hirntoddiagnostik und Patientenverfügung anhand von Aussagen erfasst, die mit „stimme voll und ganz zu“, „stimme eher zu“, „stimme eher nicht

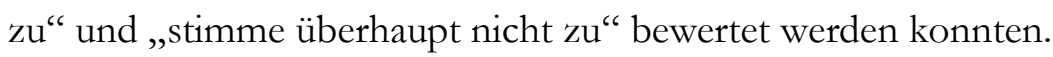

Im Folgenden werden die Bewertungen ,stimme voll und ganz $\mathrm{zu}^{\text {“ }}$ und „stimme eher zu“ als Zustimmung gewertet. Im Gegensatz dazu werden „stimme eher nicht zu“ und „stimme überhaupt nicht zu“" als Ablehnung zusammengefasst.

\subsubsection{Einstellung zur Organtransplantation und Hirntoddiagnostik}

Die Aussagen dieses Fragenblocks verdeutlichten eine überwiegend positive Einstellung zur Organtransplantation und Hirntoddiagnostik. Nahezu alle ärztlichen und pflegenden 
Mitarbeitenden (90\%) pflichteten der Aussage bei, dass die Organtransplantation eine wichtige Möglichkeit darstelle, schwerkranken Menschen zu helfen. Ebenso eindeutig hielten $89 \%$ der Befragten das von der BÄK festgelegte Verfahren zur Hirntoddiagnostik (siehe Kapitel 2.3.3.1) für geeignet, um den Hirntod als irreversiblen Ausfall aller Hirnfunktionen sicher festzustellen.

\subsubsection{Das Todeskriterium: Hirntod oder Herztod des hirntoten Patienten?}

Die beiden folgenden Aussagen, die sich mit dem Todeszeitpunkt des Menschen beschäftigen, stehen allerdings im Widerspruch zueinander (siehe Kapitel 2.3.3.1). So setzt die erste Aussage den Hirntod mit dem Tod des Menschen gleich, die darauf folgende Aussage hingegen definiert den Eintritt des Todes erst mit dem Herzstillstand des hirntoten Patienten. Da hier Mehrfachantworten zugelassen waren, war es von besonderem Interesse, das Antwortverhalten hinsichtlich des Todeskriteriums sowie der Hirntoddiagnostik genauer zu analysieren.

Diejenigen, die die Diagnostik als geeignet empfanden, haben sich unabhängig von der Profession größtenteils für den Hirntod als Tod des Menschen ausgesprochen (46 \%). 27 \% (Ärztinnen und Ärzte: 36 \%; Pflegende: $24 \%$ ) stimmten allen drei Aussagen zu: Sie beurteilten die Diagnostik als geeignet, definierten den Hirntod als den Tod des Menschen, sahen aber gleichzeitig den Menschen erst dann als wirklich tot an, wenn bei hirntoten Patienten das Herz aufhöre zu schlagen.

Von den Mitarbeitern, die das Verfahren der Hirntoddiagnostik als ungeeignet empfanden (Ärztinnen/Ärzte: $3 \%$; Pflegende: $12 \%$ ), setzten $70 \%$ den Tod des hirntoten Patienten mit dem Herztod gleich und nicht mit dem Hirntod (20\%). Dementsprechend wurde es hier als Zustimmung gewertet, wenn die Befragten jeweils einer Aussage zustimmten und die andere ablehnten. Es stimmten danach also mehr als die Hälfte die Ärztinnen und Ärzte $(53 \%)$ der Aussage zu, dass der Hirntod mit dem Tod des Menschen gleichzusetzen sei - von den Pflegenden bejahte diese Aussage ein etwas geringerer Anteil von 47\%. Die Aussage, dass hirntote Patienten erst dann wirklich tot seien, wenn das Herz nach Abschalten der Geräte zu schlagen aufgehört habe, bestätigten nur 7 \% der Ärztinnen und Ärzte und $13 \%$ der Pflegenden. Es gab jedoch Personen, die angegeben haben, dass der Hirntod mit dem Tod des Menschen gleichzusetzen sei und gleichzeitig angekreuzt haben, dass hirntote Patienten erst dann wirklich tot seien, wenn das Herz nach Abschalten der Geräte zu schlagen aufgehört habe. Unter den Ärztinnen und Ärzten waren dies 37 \%, unter den Pflegenden $30 \%$. Dieses Antwortverhalten ist so zu deuten, dass die Sicherheit des Todes trotz Hirntod erst mit dem Herztod gegeben sei. Die Mehrfachnennungen wurden daher 
hier als Plädoyer für den Herztod gewertet und der Gruppe zugeschlagen, die ausschließlich die Aussage angekreuzt haben, dass hirntote Patienten erst dann wirklich tot seien, wenn das Herz nach Abschalten der Geräte zu schlagen aufgehört habe.

Im Ergebnis sprachen sich damit $48 \%$ der Befragten für den Hirntod als Todeskriterium aus $(53 \%$ des Ärztepersonals und $47 \%$ der Pflegenden). Der Herztod war letztlich für $43 \%$ der Befragten relevant (Ärztinnen/Ärzte: 44 \%; Pflegende: $43 \%$ ). (Abb. 2)

Abbildung 2: Zustimmungen beim Thema Organspende und Hirntoddiagnostik.

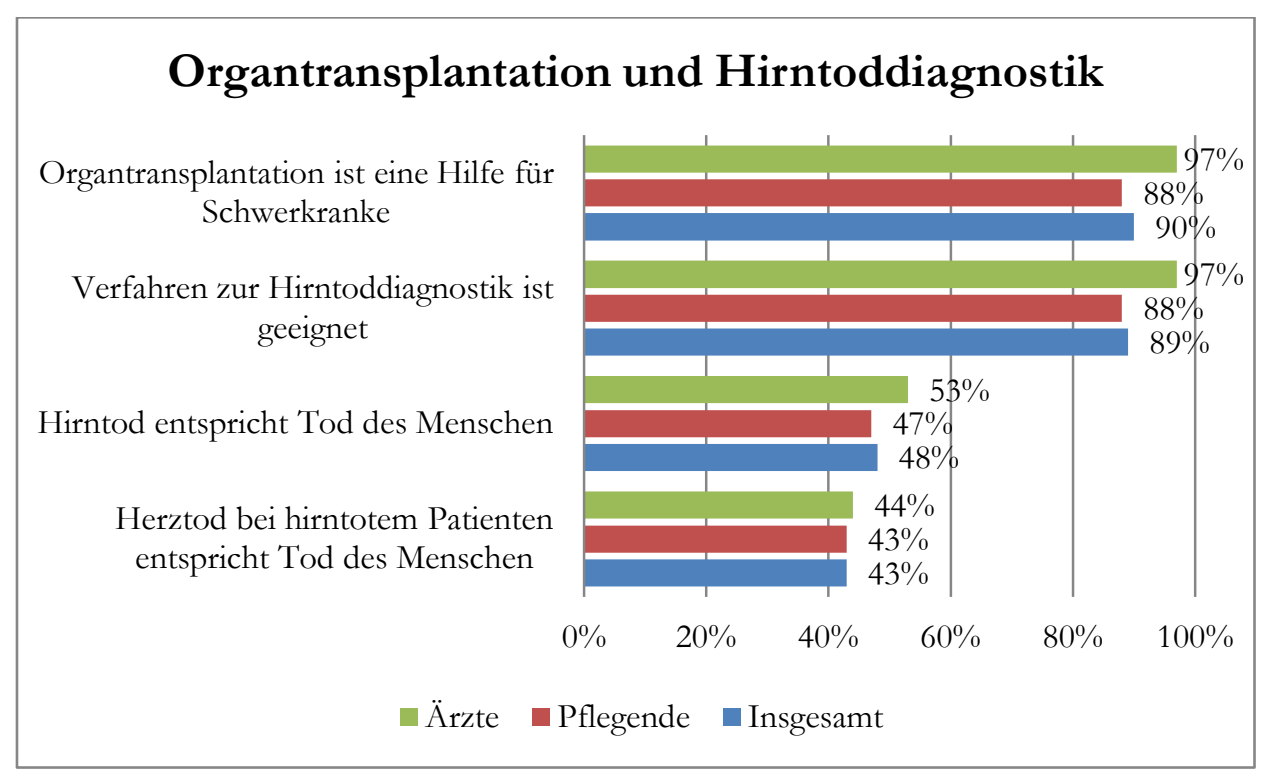

\subsubsection{Einstellung zur Patientenverfügung}

Die ärztlichen und pflegenden Teilnehmenden waren sich einig darüber, dass die Zahl der Patientenverfügungen in den letzten Jahren merklich zugenommen hat (91\%). Geteilter Meinung waren die Befragten jedoch hinsichtlich der Bedeutsamkeit und Berücksichtigung der Patientenverfügung im klinischen Alltag.

Zwar war eine deutliche Mehrheit von $79 \%$ der ärztlichen Befragten der Ansicht, dass die Patientenverfügung im klinischen Alltag in den letzten Jahren stärker berücksichtigt werde, aber nur $45 \%$ der Ärztinnen und Ärzte betrachteten die Patientenverfügung als verbindlich. Dagegen beobachteten nur 54 \% der Pflegenden eine stärkere Berücksichtigung der Patientenverfügungen bei klinischen Entscheidungen, während mehr als die Hälfte der Pflegenden (57 \%) die Patientenverfügung als verbindlich ansahen. (Abb. 3)

Die Ärztinnen und Ärzte haben die Patientenverfügungen bei ihren klinischen Entscheidungen berücksichtigt, allerdings nicht im Sinne einer verbindlichen Handlungsanweisung. Die Pflegenden hingegen betrachteten die Patientenverfügung als verbindliche Handlungsanweisung. Trotz dieser Verbindlichkeit waren die pflegenden Mitarbeiter jedoch der Mei- 
nung, dass die Patientenverfügungen in der Praxis nicht stärker als früher berücksichtigt werden. (Abb. 3)

Abbildung 3: Zustimmungen zur Einstellung zum Thema Patientenverfügung.

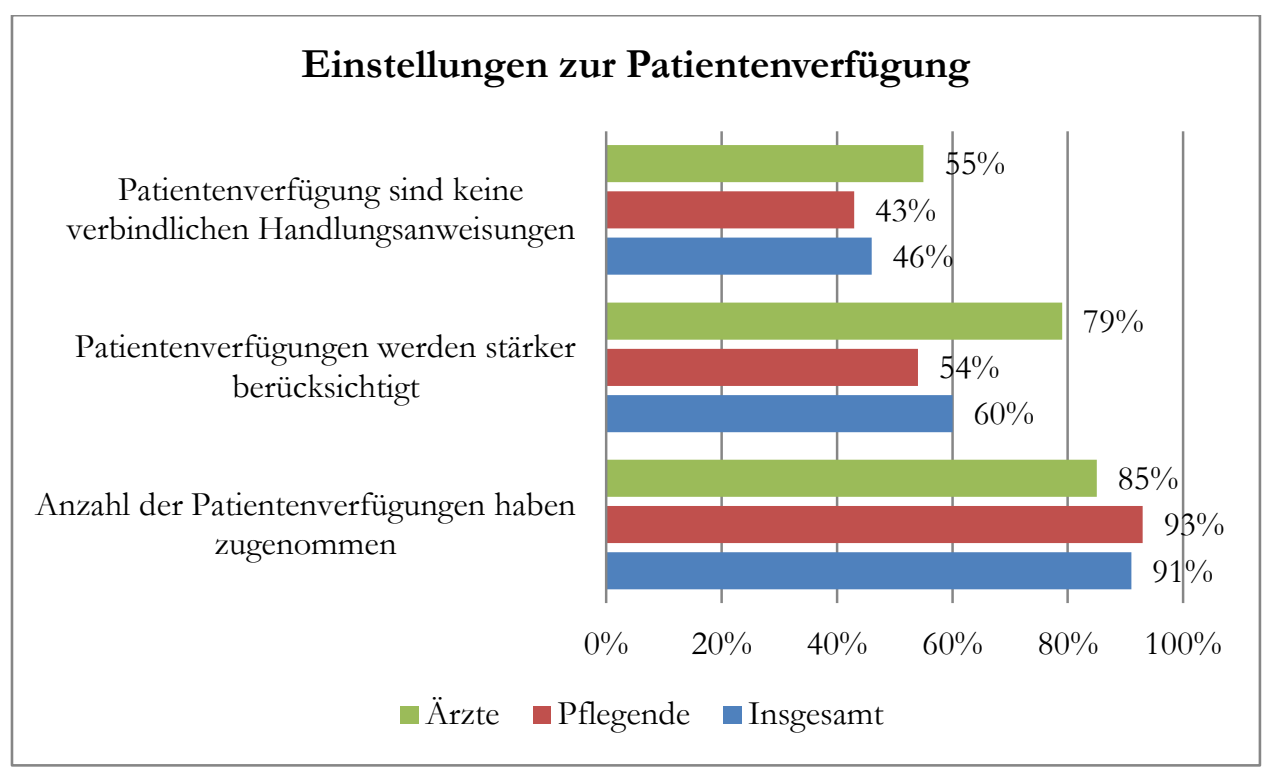

\subsubsection{Verfügen über die Dokumente}

Nach Begutachtung der Einstellung der Befragten war es nun von Interesse, ob die Teilnehmenden selbst einen Organspendeausweis oder eine Patientenverfügung besaßen.

Knapp die Hälfte aller Befragten verfügte über einen Organspendeausweis, wobei der Anteil bei den ärztlichen Mitarbeitenden mit $52 \%$ rund $10 \%$ höher lag als bei den pflegenden Mitarbeitenden (42\%). Nur knapp ein Viertel der Befragten besaß eine Patientenverfügung (Ärztinnen/ Ärzte: $17 \%$; Pflegende: $24 \%$ ).

\subsubsection{Zusammenfassung zur allgemeinen Einstellung gegenüber Organtransplantation, Hirntoddiagnostik und Patientenverfügung}

Generell sahen die Teilnehmenden die Organtransplantation sowie auch die Patientenverfügung als wichtige Bestandteile im klinischen Alltag. Trotzdem besaß der Großteil der Befragten keine Patientenverfügung, wobei knapp die Hälfte der Teilnehmenden einen Organspendeausweis hatte.

Während das Verfahren der BÄK zur Hirntoddiagnostik vom Großteil der Befragten als geeignet empfunden wurde, spaltete die Festlegung des Todeskriteriums als Herztod oder Hirntod hingegen die Ansichten der Befragten. So sahen für die Festlegung des Todeskriteriums fast die Hälfte der Befragten den Hirntod als Tod des Patienten sowie weitere $43 \%$ den Herztod des hirntoten Patienten als maßgeblich an. Auffällig war, dass außer bei der 
Beurteilung des Herztodes als Hirntod, jeweils ein geringerer Anteil der Pflegenden diesen Aussagen zustimmten.

Auch im Hinblick auf die Berücksichtigung der Patientenverfügung in der Praxis und deren Verbindlichkeit gab es Differenzen zwischen den ärztlichen und pflegenden Mitarbeitenden. Demzufolge haben die Ärztinnen und Ärzte die Patientenverfügungen zwar durchweg berücksichtigt, aber überwiegend nicht als verbindlich angesehen. Anders als die Pflegenden, die die Patientenverfügung mehrheitlich als verbindliche Handlungsanweisung betrachteten, ihre Berücksichtigung in der Praxis aber in geringerem Umfang feststellten, als von den Ärztinnen und Ärzten angegeben.

\subsection{Verhältnis von Patientenverfügung und Organspendeerklärung}

Der Hauptteil des Fragebogens bezog sich auf einen möglichen Konflikt zwischen einer Patientenverfügung und einer Organspendeerklärung. Um herauszufinden, wie in solchen Konfliktsituationen gehandelt werden würde, wurden den Befragten vier Szenarien vorgegeben. Die Teilnehmenden wurden gebeten, sich innerhalb dieser Szenarien für weitere Maßnahmen zu entscheiden.

Die Ausgangssituation aller vier Szenarien war wie folgt: Ein fiktiver Patient liegt nach einem schweren Verkehrsunfall mit einer Hirnblutung auf der Intensivstation. Er ist bewusstlos und wird maschinell beatmet. Der Patient hat eine Patientenverfügung, in der er lebenserhaltende Maßnahmen für den Fall einer schweren irreversiblen Hirnschädigung ablehnt. Diese Ausgangssituation wurde sodann wie folgt variiert:

Szenario 1: Die behandelnden Ärzte vermuten, dass der Hirntod bereits eingetreten ist.

a) Die Bereitschaft des Patienten zur Organspende ist in einen Organspendeausweis dokumentiert.

b) Die Bereitschaft des Patienten zur Organspende ist nicht bekannt.

Szenario 2: Die behandelnden Ärzte vermuten, dass der Hirntod innerhalb der nächsten Tage eintreten wird.

a) Die Bereitschaft des Patienten zur Organspende ist in einen Organspendeausweis dokumentiert.

b) Die Bereitschaft des Patienten zur Organspende ist nicht bekannt. 
Demzufolge unterscheiden sich die beiden Szenarien bezüglich des Hirntodes des fiktiven Patienten sowie bezüglich des Vorliegens eines Organspendeausweises. Im Folgenden wird jedes Szenario einzeln analysiert.

$\mathrm{Zu}$ beiden Szenarien gaben einige Personen Mehrfachantworten ab, obwohl dies bei den Fragen nicht vorgesehen war. Diese Mehrfachantworten tauchten in drei verschiedenen Konstellationen tauchten auf, wobei jeweils das Gespräch mit den Angehörigen genannt wurde. Bei der ersten Konstellation kreuzten einige Teilnehmenden gleichzeitig die Fortführung der intensivmedizinischen Maßnahmen zur Hirntoddiagnostik sowie ein Angehörigengespräch an. Zwar wäre bei dieser Antwort eine Organspende möglich, sie basierte in diesem Fall aber auf dem Gespräch mit den Angehörigen und nicht mehr auf den Dokumenten, sodass diese Mehrfachnennungen der Rubrik „Angehörigengespräch" zugeschlagen wurden. Die zweite (seltenere) Art der Mehrfachantwort beinhaltete ebenfalls das Gespräch sowie die Beendigung der intensivmedizinischen Maßnahmen. Das Gespräch lässt hier zwar eine Entscheidung zugunsten der Organspende offen, doch ermöglicht das Beenden der Maßnahmen keine Organspende, sodass diese Mehrfachnennung zum „Beenden der intensivmedizinsichen Maßnahmen" gezählt wurde. Vereinzelt wurde das Angehörigengespräch zusammen mit „Sonstiges" genannt. In diesen Fällen wurde die Antwort zu der Rubrik „Sonstiges" zugeschlagen.

\subsubsection{Vorgehen bei vermutetem Hirntod: Szenario 1}

Bei Szenario 1, in welchem vermutet wird, dass der Hirntod bereits eingetreten ist, entschieden sich die Befragten größtenteils für Antwortmöglichkeiten, die eine Realisierung der Organspende ermöglichen würden: Die Teilnehmer votierten für die Fortführung der intensivmedizinischen Maßnahmen oder für ein Gespräch mit den Angehörigen (Szenario 1a: $92 \%$; Szenario 1b: $81 \%$ ). (Abb. 4) Diese beiden Antwortmöglichkeiten implizieren die Möglichkeit einer Organspende, wobei sich die Intensität bzw. die Entscheidungsgewalt über eine Organspende unterscheidet, da sie einmal dem dokumentierten Willen des Patienten nach beim ärztlichen Personal liegt und andererseits mit den Angehörigen anhand des mutmaßlichen Patientenwillens nochmals evaluiert werden soll.

Nur eine Minderheit der Befragten entschied sich beim Szenario des vermuteten Hirntods dafür, die intensivmedizinischen Maßnahmen zu beenden und somit eine Organspende unmöglich zu machen. Allerdings spielte dabei das Vorliegen eines Organspendeausweises eine Rolle: In Szenario 1a (dokumentierte Organspendebereitschaft) entschieden sich ledig- 
lich $6 \%$ der Teilnehmenden gegen eine Organspende, bei nicht dokumentierter Organspendebereitschaft (Szenario 1b) $18 \%$ der Teilnehmenden. (Abb. 4)

5.4.1.1 Welches Vorgehen wurde für die Realisierung einer Organspende bevorzugt: Die Fortführung der intensivmedizinischen Maßnahmen oder das Gespräch mit den Angehörigen?

Um diese Frage beantworten zu können, muss man zwischen dem Vorliegen eines Organspendeausweises und einer unbekannten Bereitschaft zur Organspende differenzieren. Sofern die Bereitschaft vorlag (Szenario 1a) favorisierte die Hälfte der Teilnehmenden (50 \%) die Fortführung der intensivmedizinischen Maßnahmen, um eine Hirntoddiagnostik durchführen zu können. $42 \%$ der Befragten wollte zunächst ein Gespräch mit den Angehörigen führen. Genau anders herum gestaltete es sich, wenn die Bereitschaft zur Organspende unbekannt war (Szenario 1b): Nur $28 \%$ der Teilnehmenden sprach sich dann für die Fortführung der intensivmedizinischen Maßnahmen mit einem anschließenden Angehörigengespräch aus. Mehr als die Hälfte der Befragten (53 \%) wollte in dieser Konstellation (Szenario 1b) das weitere Vorgehen (einschließlich der Frage einer möglichen Organspende) direkt mit den Angehörigen besprechen.

Diese unterschiedliche Gewichtung der Rolle der Angehörigen, abhängig von einer dokumentierten Bereitschaft zur Organspende, spiegelte sich auch im Vergleich zwischen den beiden Professionen wider. Im Gesamten sprach sich zwar eine deutliche Mehrheit der Ärztinnen und Ärzte (Szenario 1a: 100 \%; Szenario 1b: 83 \%) sowie auch der Pflegenden (Szenario 1a: 90 \%; Szenario 1b: $80 \%$ ) für die Antwortmöglichkeiten aus, die eine Organspende realisieren würden, dies jedoch mit variierender Intensität (Abb.4): Es entscheiden sich bei Szenario 1a (dokumentierte Organspendebereitschaft) mehr Ärztinnen und Ärzte (66\%) als Pflegende (45\%) für die Durchführung der Hirntoddiagnostik. Die Pflegenden votierten hingegen zu gleichen Teilen einerseits für die Fortführung der intensivmedizinischen Maßnahmen (45\%) und andererseits für das Gespräch mit den Angehörigen, um das weitere Vorgehen festzulegen (Ärztinnen und Ärzte: 35 \%; Pflegende: 45 \%). (Abb. 4) Sofern die Bereitschaft zur Organspende unbekannt war (Szenario 1b), ergaben sich kaum Unterschiede zwischen den beiden Professionen: Jeweils mehr als die Hälfte sowohl der ärztlichen ( $52 \%$ ) als auch der pflegenden Mitarbeiter (53\%) favorisierten bei Szenario 1b (unbekannte Organspendebereitschaft) das Angehörigengespräch, um das weitere Vorgehen mit diesen zu besprechen. Knapp ein Drittel der Ärztinnen und Ärzte (31\%) sowie $27 \%$ der Pflegenden entschieden sich für die Fortführung der intensivmedizinischen Maß- 
nahmen zur Hirntoddiagnostik und erst im Anschluss daran ein Gespräch mit den Angehörigen.

Abbildung 4: Es wird vermutet, dass der Hirntod beim Patienten bereits eingetreten ist. Eine Organspendebereitschaft ist dokumentiert (Szenario 1a).

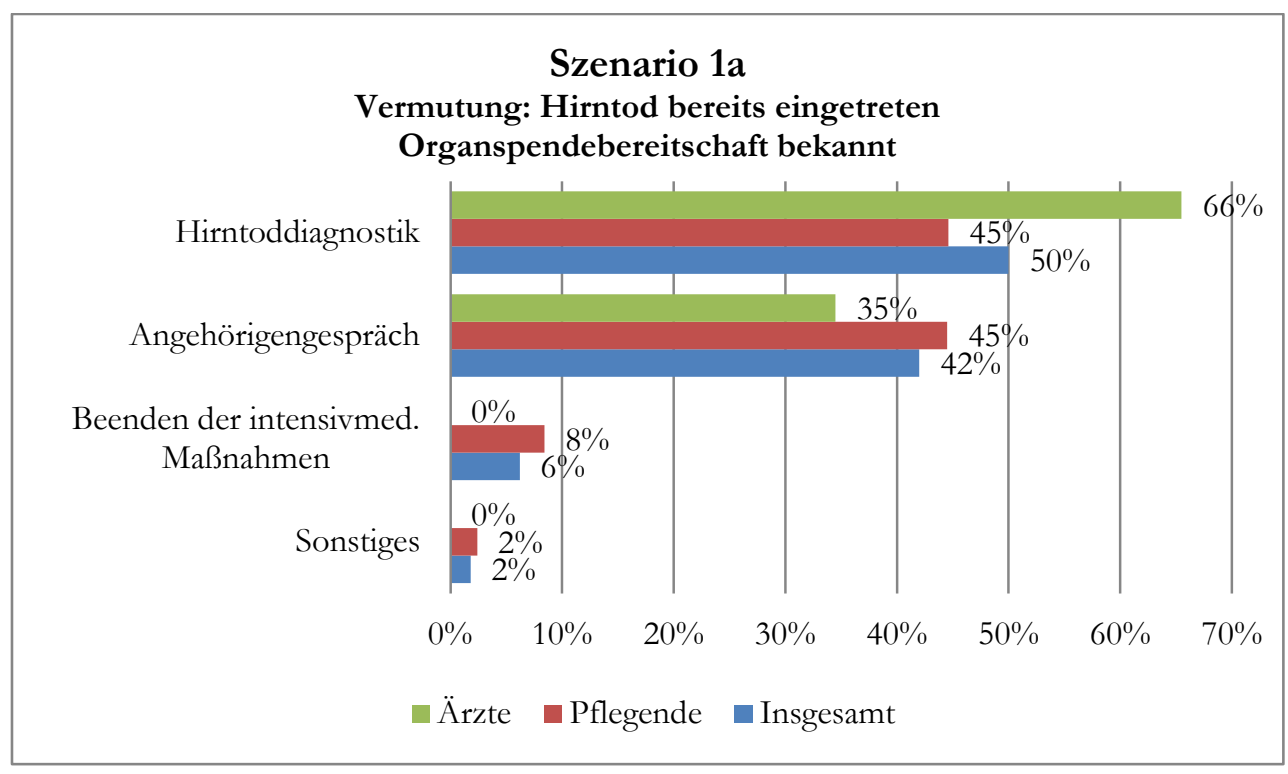

\subsubsection{Wer votierte gegen eine Organspende?}

Wie bereits geschildert, wählte insgesamt nur ein sehr geringer Anteil der Befragten beim vermuteten Hirntod des fiktiven Patienten die Beendigung der intensivmedizinischen Maßnahmen, wonach keine Organspende mehr möglich wäre. Beim vermuteten Hirntod und gleichzeitig fraglicher Bereitschaft des Patienten zur Organspende (Szenario 1b) entschieden sich generell mehr Teilnehmer für das Beenden der Maßnahmen als bei Szenario 1a (siehe Kapitel 5.4.1).

Auffällig war hierbei, dass sich mehr pflegende als ärztliche Mitarbeiter für das Beenden der Maßnahmen entschieden: Bei bekannter Organspendebereitschaft (Szenario 1a) sprachen sich $8 \%$ der Pflegenden und kein ärztlicher Mitarbeiter für das Beenden aus. Bei Szenario $1 \mathrm{~b}$, in dem die Bereitschaft zur Spende unbekannt war, wählten generell mehr als doppelt so viele Pflegende (21\%) dieses Vorgehen, von den Ärztinnen und Ärzten nur $10 \%$. (Abb. 5) 
Abbildung 5: Es wird vermutet, dass der Hirntod beim Patienten bereits eingetreten ist. Eine Organspendebereitschaft ist unbekannt (Szenario 1b).

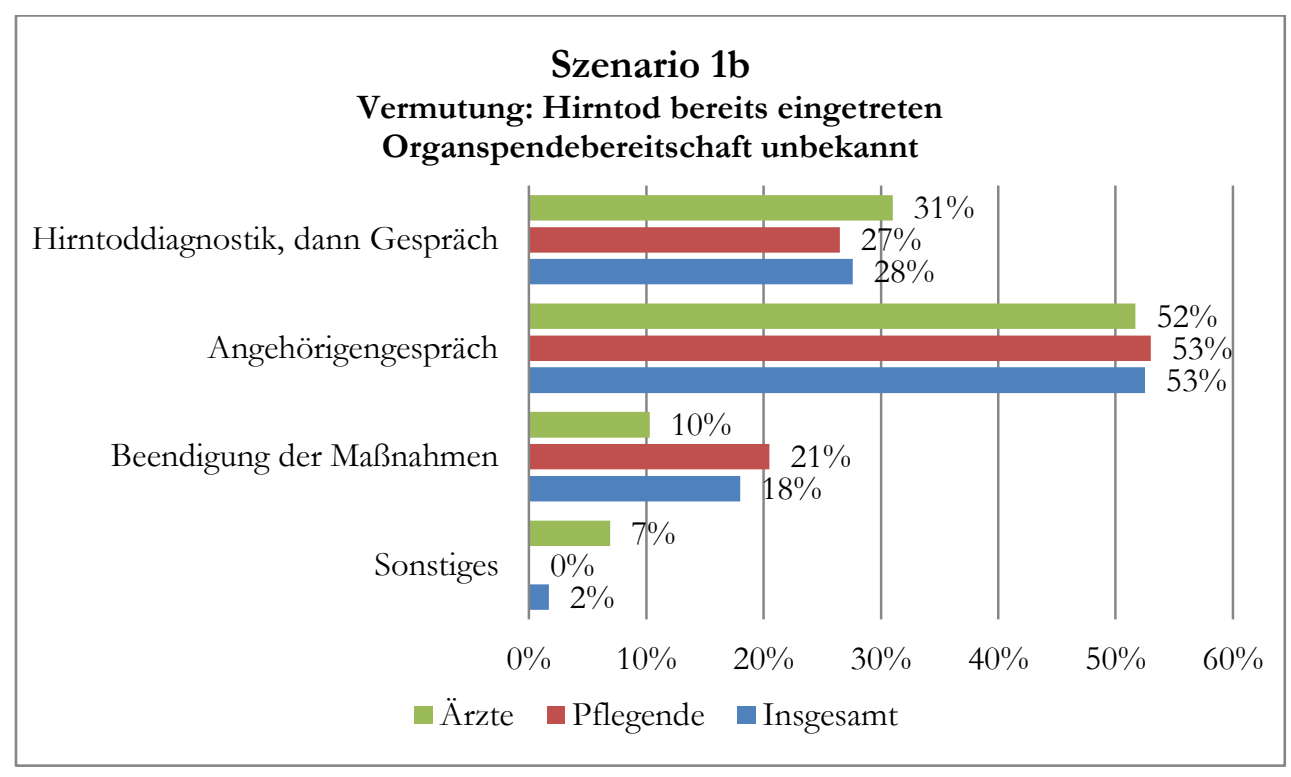

In der nachfolgenden Tabelle sind die Aussagen aufgeführt, die bei „Sonstiges“ festgehalten wurden. (Tabelle 4)

Tabelle 4: Aussagen bei „Sonstiges" des ersten Szenarios

\begin{tabular}{|l|l|}
\hline Organspendebereitschaft bekannt & Organspendebereitschaft unbekannt \\
\hline $\begin{array}{l}\text { Wenn Hirnschädigung irreversibel und schwer, } \\
\text { muss die Therapie eingestellt werden } \\
\text { Angehörige/Patientenverfügung }\end{array}$ & $\begin{array}{l}\text { intensivmedizinische Maßnahmen bis } \\
\text { Angehörigengespräch fortführen } \\
\end{array}$ \\
$\begin{array}{l}\text { Organspende ansprechen, lebenserhaltende } \\
\text { Maßnahmen beenden }\end{array}$ \\
\hline
\end{tabular}

\subsubsection{Vorgehen bei Vermutung eines baldig eintretenden Hirntods: Szenario 2}

Wie bei Szenario 1 (siehe Kapitel 5.4.1), entschied sich die Mehrheit der Teilnehmenden auch beim zweiten Szenario, in dem vermutet wird, dass der Hirntod des Patienten innerhalb der nächsten Tage eintreten wird, für die Möglichkeit der Realisierung einer Organspende des Patienten. Dies jedoch abhängig von der vorliegenden Bereitschaft zur Organspende mit unterschiedlicher Ausprägung: Sofern die Bereitschaft zur Organspende unklar war (Szenario 2b) votierten insgesamt etwas weniger der Befragten (70 \%) für die begrenzte Fortführung der intensivmedizinischen Maßnahmen oder das Gespräch mit den Angehörigen, als bei Szenario 2a (dokumentierte Organspendebereitschaft: 84 \%).

Das Vorliegen eines Organspendeausweises hatte also wie schon bei Szenario 1 (vermuteter Hirntod) Auswirkungen auf das Antwortverhalten. Dementsprechend wählten die Befrag- 
ten bei vorliegendem Ausweis (Szenario 2a) einerseits die begrenzte Fortführung der intensivmedizinischen Maßnahmen, um den Eintritt des Hirntodes abzuwarten (41\%), und andererseits das Gespräch mit den Angehörigen (43\%). (Abb. 6) Diese Verteilung ändert sich bei Szenario 2b (unbekannte Organspendebereitschaft): Zwar votierten ebenfalls 40 \% der Teilnehmer für ein Gespräch mit den Angehörigen, allerdings wählten nur $30 \%$ die begrenzte Fortführung der intensivmedizinischen Maßnahmen mit anschließendem Angehörigengespräch. Ein ebenso großer Anteil, der sich für die Fortführung der Maßnahmen entschied, sprach sich bei Szenario $2 b$ (unbekannte Organspendebereitschaft) für eine Beendigung der intensivmedizinischen Maßnahmen (29\%) aus. Das Votum gegen eine Organspende fiel generell bei dem baldig eintretenden Hirntod des fiktiven Patienten (Szenario 2) deutlicher aus als bei dem bereits vermuteten Hirntod (Szenario 1), vor allem (wie bereits bei Szenario 1) sobald die Bereitschaft zur Organspende gleichzeitig noch unklar war (Szenario 2b). Wiederum ergaben sich signifikante Unterschiede zwischen den ärztlichen und pflegenden Mitarbeitern, dahingehend, dass sich, wie schon bei Szenario 1, mehr Pflegende als Ärztinnen und Ärzte gegen eine Organspende aussprachen (siehe Kapitel 5.4.2.1).

\subsubsection{Ergaben sich Diskrepanzen zwischen den beiden Professionen?}

Wie schon beim vorangegangenen Szenario (Szenario 1: vermuteter Hirntod) war auch bei Szenario 2 (baldig eintretender Hirntod) auffällig, dass sich die Ärztinnen und Ärzte eher für die begrenzte Fortführung der intensivmedizinischen Maßnahmen und gegen eine Beendigung der intensivmedizinischen Maßnahmen aussprachen als die Pflegenden.

Besonders deutlich wird diese Diskrepanz zwischen den Professionen beim Szenario 2a, in dem eine Organspendebereitschaft dokumentiert ist: Während die Ärztinnen und Ärzte die begrenzte Fortführung der intensivmedizinischen Maßnahmen (57\%) favorisierten, wählten nur $35 \%$ der pflegenden Mitarbeitenden dieses Vorgehen. Zwar entschieden sich genau so viele Pflegende (43\%) wie ärztliche Mitarbeiter (43\%) für das Gespräch mit den Angehörigen, allerdings entschieden sich $19 \%$ der Pflegenden gegen eine Organspende. Von den ärztlichen Mitarbeitenden sprach sich keiner für eine Beendigung der intensivmedizinischen Maßnahmen aus. (Abb. 6) 
Abbildung 6: Es wird vermutet, dass der Hirntod innerhalb der nächsten Tage eintreten wird. Eine Organspendebereitschaft ist bekannt (Szenario 2a).

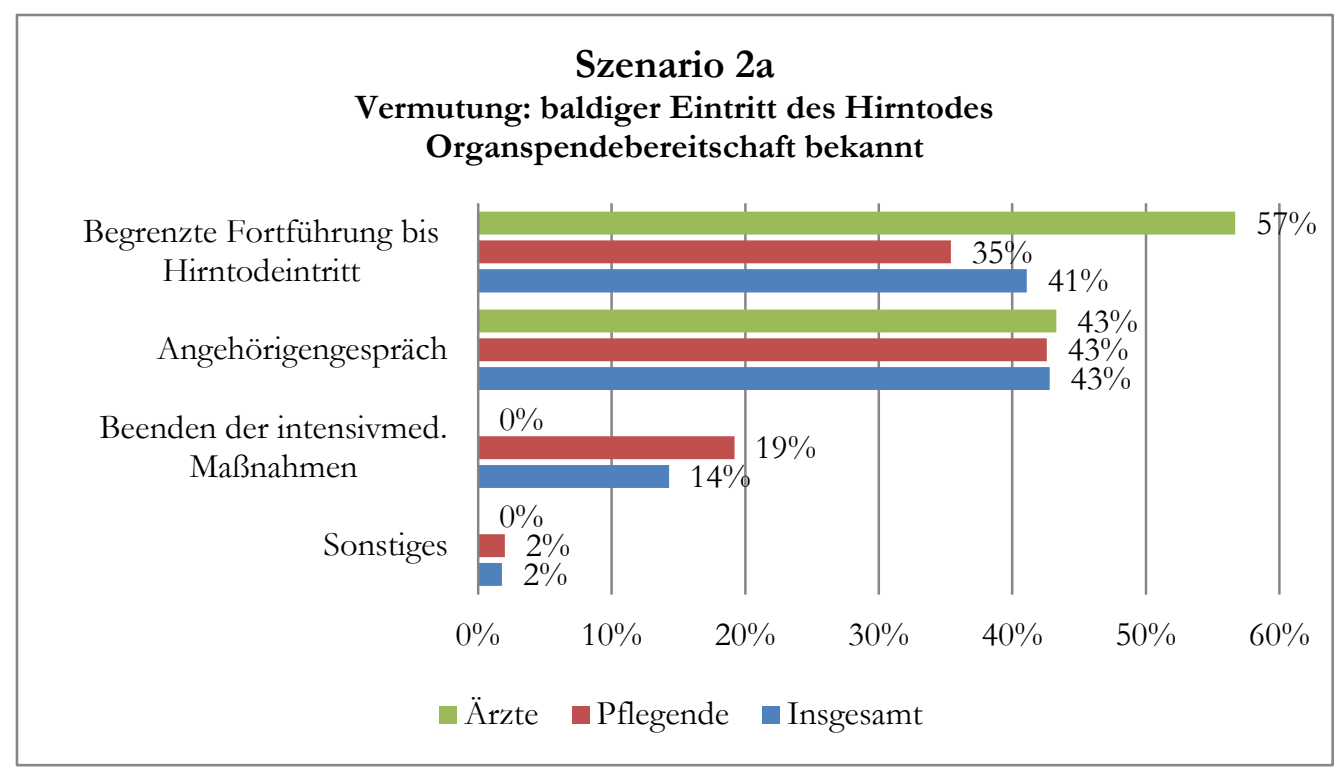

Sobald die Breitschaft zur Organspende unbekannt war (Szenario 2b), wurde von den Ärztinnen und Ärzten nicht mehr das Fortführen der intensivmedizinischen Maßnahmen favorisiert (Szenario 2b: 31 \%), sondern ein Gespräch mit den Angehörigen (53\%). Die Pflegenden, die schon bei Szenario 2a (dokumentierte Organspendebreitschaft) mehrheitlich das Angehörigengespräch wählten (43\%), taten dies auch bei unbekannter Organspendebreitschaft (Szenario 2b), allerdings lediglich zu einem Drittel (35\%). Zu fast gleichen Anteilen entschieden die pflegenden Mitarbeiter sich einerseits für die begrenzte Fortführung der intensivmedizinischen Maßnahmen (Ärztinnen und Ärzte: 31 \%; Pflegende: $29 \%$ ) und andererseits für das Beenden der intensivmedizinischen Maßnahmen (31\%). Unter den Ärztinnen und Ärzten votierten nur $17 \%$ gegen eine Organspende. (Abb. 7) Auch bei Szenario 2a (dokumentierte Organspendebreitschaft) wurde deutlich, dass vor allem die Pflegenden eine Organspende nicht befürworteten, da sich im Gegensatz zu keinem ärztlichen Mitarbeiter $19 \%$ der Pflegenden für das Beenden der intensivmedizinischen Maßnahmen aussprachen. 
Abbildung 7: Es wird vermutet, dass der Hirntod innerhalb der nächsten Tage eintreten wird. Eine Organspendebereitschaft ist unbekannt (Szenario 2b).

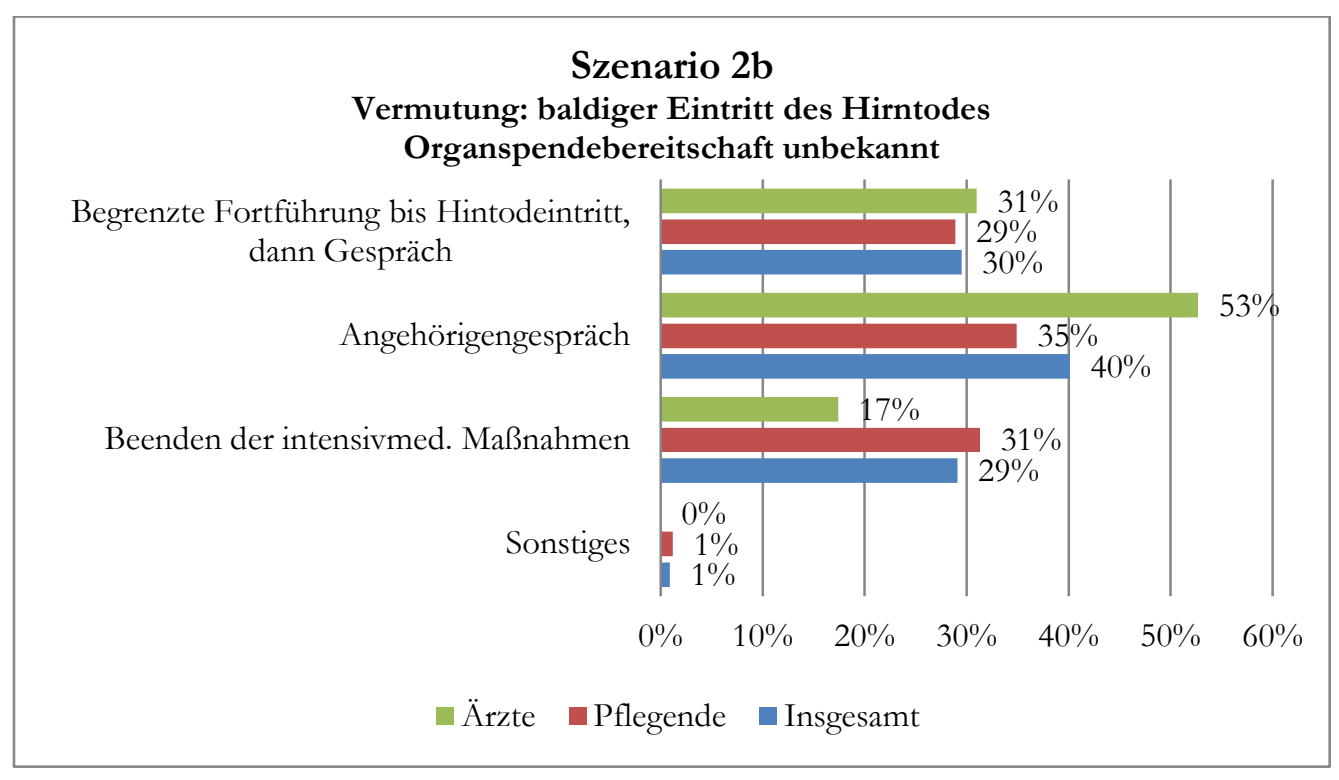

5.4.2.2 Wie lange wäre eine Fortführung der intensivmedizinischen Maßnahmen für die Befragten vertretbar?

Sowohl bei bekannter Organspendebreitschaft (Szenario 2a), als auch bei unbekannter Bereitschaft (Szenario 2b), konnten die Befragten eine Fortführung der intensivmedizinsichen Maßnahmen wählen, allerdings für einen begrenzten Zeitraum. Generell sprachen sich, wie bereits erläutert (siehe Kapitel 5.4.2.1) vor allem bei Szenario 2a (dokumentierte Organspendebreitschaft) die ärztlichen Mitarbeiter für die Fortführung der Maßnahmen aus, um den Eintritt des Hirntodes abzuwarten.

Von Interesse war nun zu beurteilen, wie lange diese Fortführung der Maßnahmen für die Befragten vertretbar war. Für die Ärztinnen und Ärzte war die Fortführung im Mittel für maximal 3,6 Tage diskutabel, für die Pflegenden rund einen Tag länger (Mittelwert: 4,8 Tage). Neben dieser konkreten Zeitangabe wurde von der Hälfte der ärztlichen Befragten (Ärztinnen und Ärzte: $52 \%$; Pflegende: $14 \%$ ) auch „Sonstiges“ angekreuzt und oftmals ergänzt, dass es eine individuelle, situationsgebundene und fallabhängige Entscheidung sei. (Tabelle 5) Die im Freitext aufgeschriebenen Antworten sind in Tabelle 5 aufgeführt. 
Tabelle 5: Aussagen bei „Sonstiges" der Frage nach dem ethisch vertretbaren Zeitraum

\section{Ethisch vertretbarer Zeitraum für Fortführung der intensivmedizinischen Maßnahmen}

zügige Hirntoddiagnostik, ohne unnötige Verschleppung

abhängig vom Zustand des Patienten

abhängig von Entscheidung zur Organentnahme

bis zur Klärung/Hirntoddiagnostik

alle 2-3 Tage neue Evaluation-> neue Entscheidung

bis Diagnostik abgeschlossen

eine Woche, dann Reevaluation

Einbeziehung eines Ethikkomitees

CCT- Perfusiongebundene Aussage

bis $100 \%$ sicher ist, dass eine irreversible cerebrale Schädigung eingetreten ist/bis Angehörige sich verabschiedet haben

wenn in 3-4 Tage bei über zweimaliger Hirntoddiagnostik noch nicht alle Kriterien erfüllt sind

Individuell $(4 \mathrm{x})$

abhängig von Komplikationen im Verlauf

fallabhängig

weitere Diagnostik bzgl. Schwere der Hirnverletzung

solange Pat und Angehörige benötigen

solange, bis mit Angehörigen weiteres Prozedere besprochen wurde

soviel, wie zur Entscheidung benötigt wird

bis Diagnostik abgeschlossen

\subsubsection{Zusammenfassung zum Verhältnis von Patientenverfügung und Organspendeerklärung}

Zusammenfassend kann man feststellen, dass die Teilnehmenden durchweg ein Vorgehen wählen würden, das eine Organspende prinzipiell ermöglicht. Dies je nach Vorliegen eines Organspendeausweises, den unterschiedlichen Szenarien und auch der Profession in unterschiedlicher Intensität.

Im Gesamten betrachtet, wurde nur bei Szenario 1a (vermuteter Hirntod; dokumentierte Organspendebreitschaft) die Fortführung der intensivmedizinischen Maßnahmen dem Gespräch mit den Angehörigen vorgezogen. Sonst favorisierten die Teilnehmer sowohl bei Szenario 1b (vermuteter Hirntod, unbekannte Organspendebereitschaft) als auch bei dem baldig eintretendem Hirntod des Patienten (Szenario 2) das Angehörigengespräch, wobei $\mathrm{zu}$ beachten ist, dass bei Szenario 2a (baldig eintretender Hirntod; dokumentierte 
Organspendebreitschaft) die Fortführung der intensivmedizinischen Maßnahmen zu fast gleichen Teilen wie das Gespräch mit den Angehörigen gewählt wurde.

Ebenfalls war im Vergleich zwischen den beiden Szenarien auffällig, dass die Befragten deutlicher gegen eine Organspende votierten, wenn die Bereitschaft zur Organspende unklar war (Szenarien 1b und 2b) und wenn der Hirntod des Patienten erst innerhalb der nächsten Tage eintreten würde (Szenario 2).

Hinsichtlich der Professionen wurde deutlich, dass sich die Pflegenden insgesamt zurückhaltender für die Hirntoddiagnostik bzw. für das Fortführen der intensivmedizinischen Maßnahmen aussprachen. Eher votierten sie für das Gespräch mit den Angehörigen - auBer in Szenario 1a (vermuteter Hirntod; dokumentierte Organspendebereitschaft), wo sich die Pflegenden zu gleichen Teilen für ein Angehörigengespräch und eine Hirntoddiagnostik entschieden. Die ärztlichen Mitarbeiter hingegen favorisierten bei einer dokumentierten Organspendebereitschaft des Patienten (Szenario 1a und 2a) die Fortführung der intensivmedizinischen Maßnahmen und bei unklarer Bereitschaft zur Organspende (Szenario 1b und 2b) das Gespräch mit den Angehörigen. Weder bei vermutetem (Szenario 1), noch bei baldig eintretendem Hirntod sprachen sich die Ärztinnen und Ärzte gegen eine Organspende aus, allerdings nur wenn die Bereitschaft des Patienten zur Organspende dokumentiert war (Szenario 1a und 2a). Im Gegensatz zu den pflegenden Mitarbeitern, die sich in allen Konstellationen für das Beenden der Maßnahmen entschieden. (Abb. 8)

Abbildung 8: Entscheidung für die Maßnahme des Beendens der intensivmedizinischen Maßnahmen bei den unterschiedlichen Szenarien.

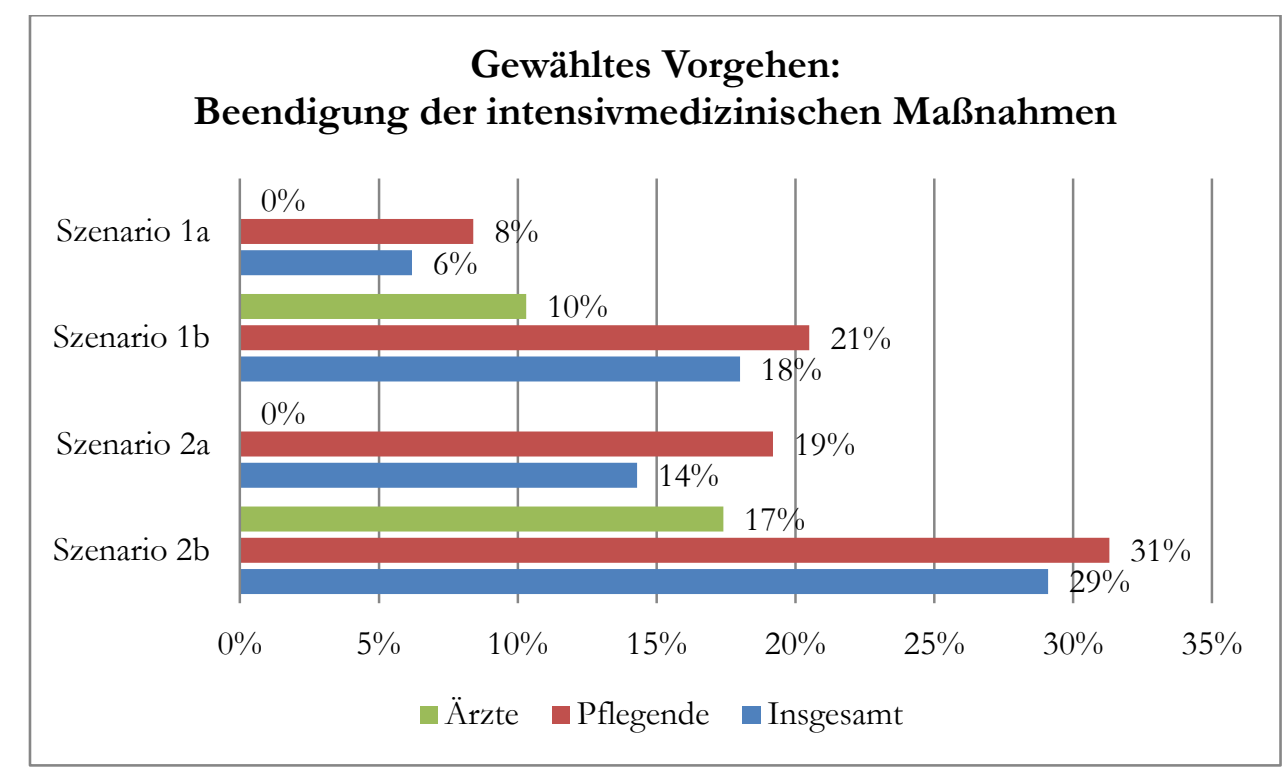




\subsubsection{Korrelation zwischen grundsätzlichen Einstellungen der Befragten zu ihren Handlungsentscheidungen}

Mit Hilfe einer zweidimensionalen Häufigkeitsverteilung wurde eine Korrelation zwischen der grundsätzlichen Einstellung der Teilnehmenden zur Patientenverfügung und Organspende sowie ihren favorisierten Vorgehen bei den unterschiedlichen Szenarien hergestellt. Die Einstellung der Teilnehmer wurde dazu anhand des Antwortverhaltens bezüglich der Thesen zu den Themen Organtransplantation, Hirntoddiagnostik und Patientenverfügung abgelesen (siehe Kapitel 5.3). Die dadurch festgestellte Einstellung der Befragten wurde dann mit dem Antwortverhalten bei den Szenarien in Verbindung gesetzt. Hat also jemand, der die Hirntoddiagnostik als ungeeignet empfand, sich eher gegen eine Organspende des in den Szenarien dargestellten fiktiven Patienten entschieden, oder doch dafür?

Auffällig war dabei, dass der Anteil derer, die eine Beendigung der intensivmedizinischen Maßnahmen wählten, höher war, sobald den Thesen zur Organspende, Hirntoddiagnostik und der Patientenverfügung nicht zugestimmt wurde. Sonst ergaben sich im Antwortverhalten bei den Szenarien kaum Unterschiede zwischen den Daten ohne Korrelation, wo also kein Zusammenhang zwischen der Einstellung und dem Antwortverhalten erstellt wurde, und denen mit Korrelation. Deshalb wird im Folgenden nur auf Thesen eingegangen, bei denen in den Szenarien signifikante Unterschiede zu dem Antwortverhalten ohne Korrelation herrschten.

5.4.4.1 Aussage: „Organtransplantation ist eine wichtige Möglichkeit, um schwerkranken Menschen zu helfen“"

Wie bereits in Kapitel 5.4.3 ausgeführt, wählten die Teilnehmenden mehrheitlich ein Vorgehen, das eine Organspende ermöglichen würde. Auch in Verbindung mit der Aussage, dass die Organtransplantation eine wichtige Möglichkeit darstelle, um schwerkranken Menschen zu helfen.

Dabei wurde aber deutlich, dass die Kritiker der Organtransplantation die Fortführung der intensivmedizinischen Maßnahmen zurückhaltender wählten als die Befürworter, das Beenden der intensivmedizinischen Maßnahmen jedoch deutlicher. Darüber hinaus gab es lediglich bei Szenario $2 \mathrm{a}$ (baldig eintretender Hirntod; dokumentierte Organspendebereitschaft) signifikante Unterschiede hinsichtlich des Vorgehens, das sonst dem Antwortverhalten in den Szenarien entsprach, unabhängig von der Einstellung der Befragten zur Organtransplantation sowie unabhängig vom Vorliegen eines Organspendeausweises oder den Antworten der Teilnehmenden ohne Bezug auf die Einstellung zur Organtransplantation. Sowohl die Befürworter der These, dass die Organ- 
transplantation eine Hilfe für Schwerkranke darstelle, als auch die Kritiker, sprachen sich bei Szenario 2a insgesamt deutlicher für die begrenzte Fortführung der intensivmedizinischen Maßnahmen als für das Gespräch mit den Angehörigen aus. Demzufolge wählten $67 \%$ der Teilnehmenden, die eine Organspende grundsätzlich befürworteten, in Szenario 2a (baldig eintretender Hirntod; dokumentierte Organspendebereitschaft) die intensivmedizinische Fortführung über einen begrenzten Zeitraum sowie auch die Hälfte der Kritiker (50\%). Nur jeweils knapp $20 \%$ (Zustimmende: $18 \%$; Ablehnende: $20 \%$ ) wollten ein Gespräch mit den Angehörigen des Patienten führen, unabhängig von der Einstellung zur Organtransplantation, also ohne Korrelation, waren es doppelt so viele (43\%). (Abb. 9)

Abbildung 9: Betrachtet wurde die Aussage, dass die Organtransplantation eine wichtige Hilfe für Schwerkranke darstelle in Verbindung mit Szenario 2a (baldiger Hirntodeintritt / dokumentierte Organspendebereitschaft)

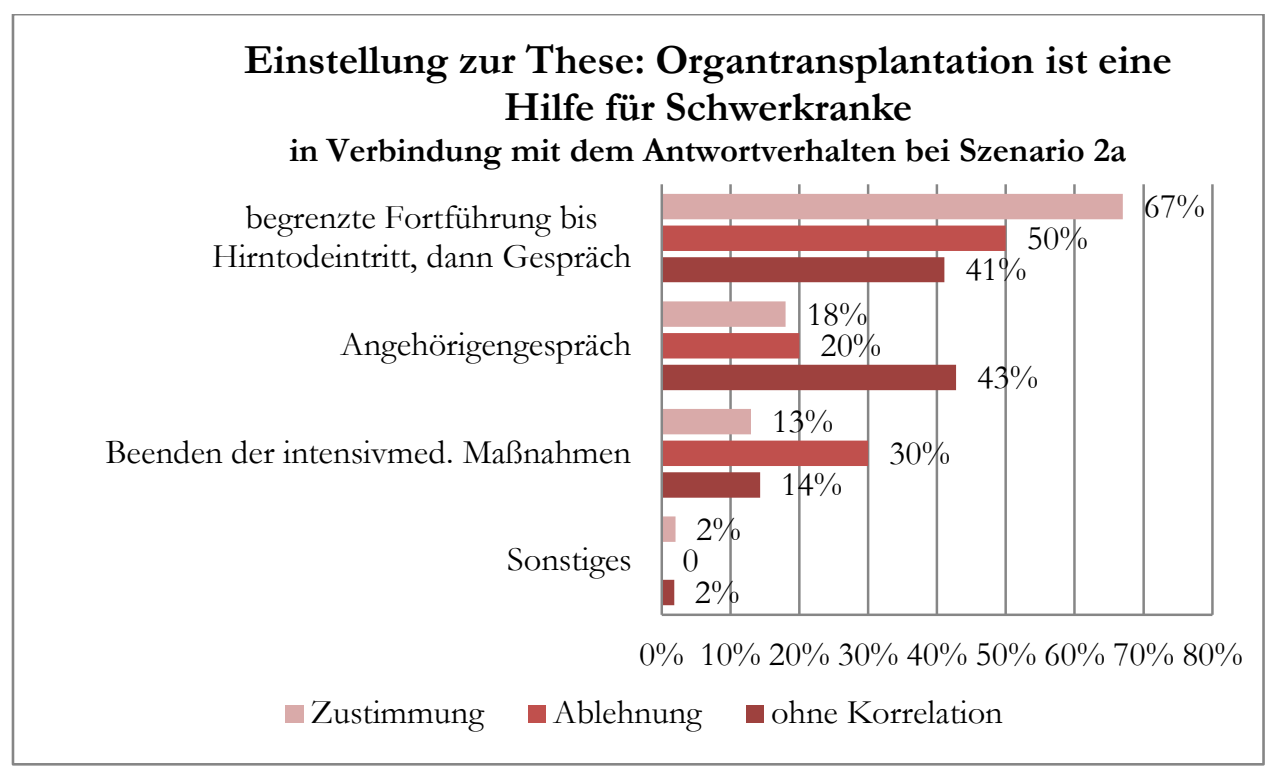

Neben diesen Auffälligkeiten, war das Votum gegen die Organspende von besonderem Interesse. Auffällig war dabei, dass diejenigen, die eine Organspende grundsätzlich ablehnten, vermehrt gegen eine Organspende votierten. Unabhängig vom Vorliegen eines Organspendeausweises und auch unabhängig von den unterschiedlichen Konstellationen in den Szenarien. Jeweils knapp ein Drittel derer (30\%), die eine Organtransplantation nicht als Hilfe für Schwerkranke ansahen, sprachen sich trotz dokumentierter Organspendebereitschaft (Szenario 1a und 2a) für das Beenden der Maßnahmen und damit gegen eine Organspende aus. Von den Befürwortern der Organtransplantation als Hilfe waren es in Szenario 1a nur $4 \%$ und in Szenario 2a lediglich $13 \%$. Sobald die Bereitschaft zur Organspende unklar war (Szenario 1b und 2b), votierten sogar jeweils $40 \%$ der Teil- 
nehmenden, die eine Organtransplantation nicht als Hilfe einschätzten, in beiden Szenarien gegen eine Organspende. Zwar stimmten auch Befürworter der These für das Beenden, aber lediglich $16 \%$ in Szenario $1 \mathrm{~b}$ (vermuteter Hirntod; unbekannte Organspendebereitschaft) und $28 \%$ bei baldigem Hirntodeintritt (Szenario 2b). (Abb.10 ) Die Prozentsätze der Teilnehmenden, die eine Organtransplantation als Hilfe ansahen, stimmen mit dem überein, was die Befragten unabhängig von ihrer Einstellung zur Organtransplantation entschieden hatten.

Abbildung 10: Korrelation zwischen der Aussage, dass die Organtransplantation eine Hilfe für Schwerkranke ist und dem gewählten Vorgehen des Beendens der intensivmedizinischen Maßnahmen im Zusammenhang mit allen Szenarien.

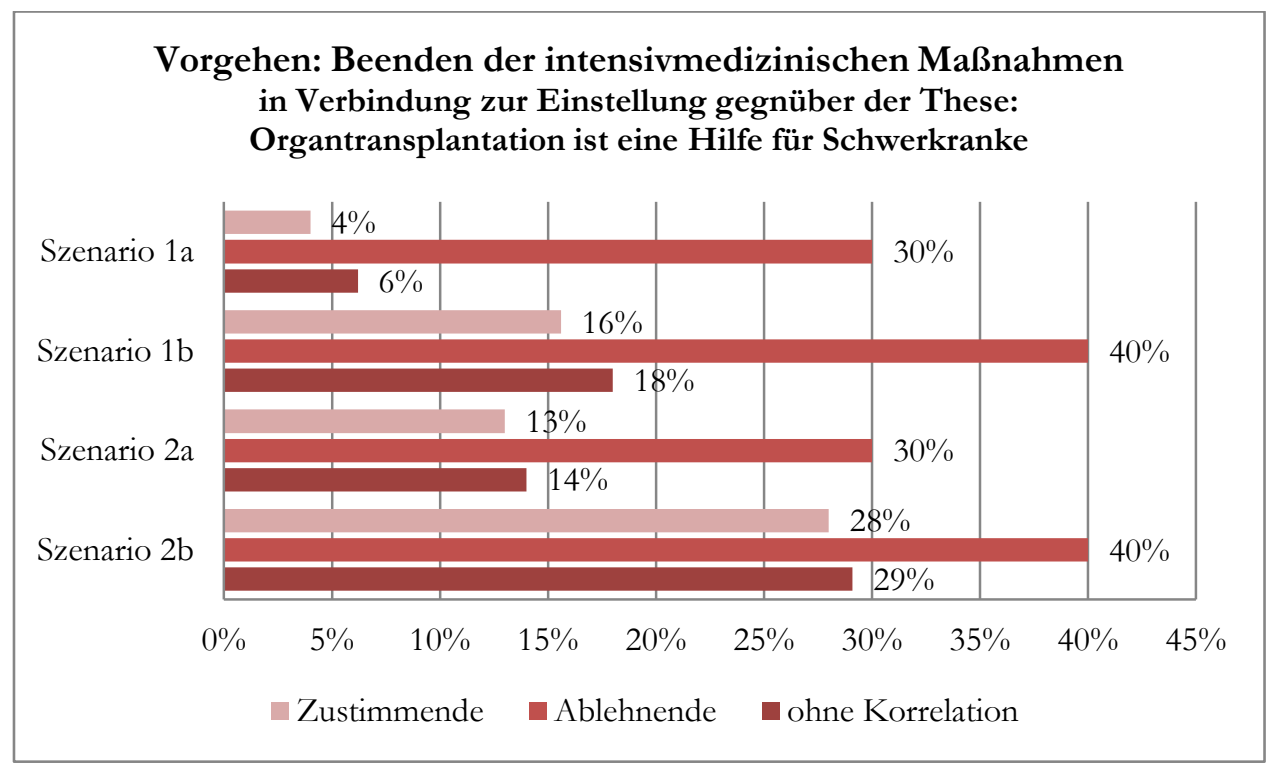

5.4.4.2 Aussage: „Das von der BÄK festgelegte Verfahren zur Hirntoddiagnostik ist geeignet, den Hirntod als irreversiblen Ausfall aller Hirnfunktionen sicher festzustellen“

Die Teilnehmenden, die das Verfahren der BÄK zur Hirntoddiagnostik (siehe Kapitel 2.3.3.1) als geeignet empfanden, entschieden größtenteils so, wie die Befragten ohne Korrelation, also unabhängig von ihrer Einstellung zur Diagnostik. Allerdings war auch bei dieser These auffällig, dass die Kritiker des Verfahrens sich in den Szenarien etwas anders entschieden als die Befürworter des Verfahrens.

Dementsprechend favorisierten die Kritiker der Hirntoddiagnostik bei den Szenarien das Gespräch mit den Angehörigen (Szenario 1a: 50 \%; Szenario 1b: 58 \%; Szenario 2a: 42 \%), abgesehen von Szenario 2b, wo das Beenden der intensivmedizinischen Maßnahmen gewählt wurde (42\%). (Abb. 11) Ohne die Berücksichtigung der Einstellung zur Hirntoddiagnostik entschied sich die Mehrheit für ein Angehörigengespräch (Szenario 1b: 53 \%; Sze- 
nario 2a: $43 \%$; Szenario 2b: $40 \%$ ), wobei einerseits bei Szenario 1a (vermuteter Hirntod; unbekannte Organspendebereitschaft) die Fortführung der intensivmedizinsichen Maßnahmen favorisisert wurde $(50 \%)$ und andererseits bei Szenario 2a (baldig eintretender Hirntod; dokumentierte Organspendebereitschaft) die Gewichtung zwischen der Fortführung der Maßnahmen (41\%) und dem Angehörigengespräch (43\%) nahezu gleich waren.

Abbildung 11: Betrachtet wurde die Aussage, dass das Verfahren der BÄK zur Hirntoddiagnostik geeignet ist in Verbindung mit Szenario 1a (eingetretender Hirntod/ dokumentierte Organspendebereitschaft).

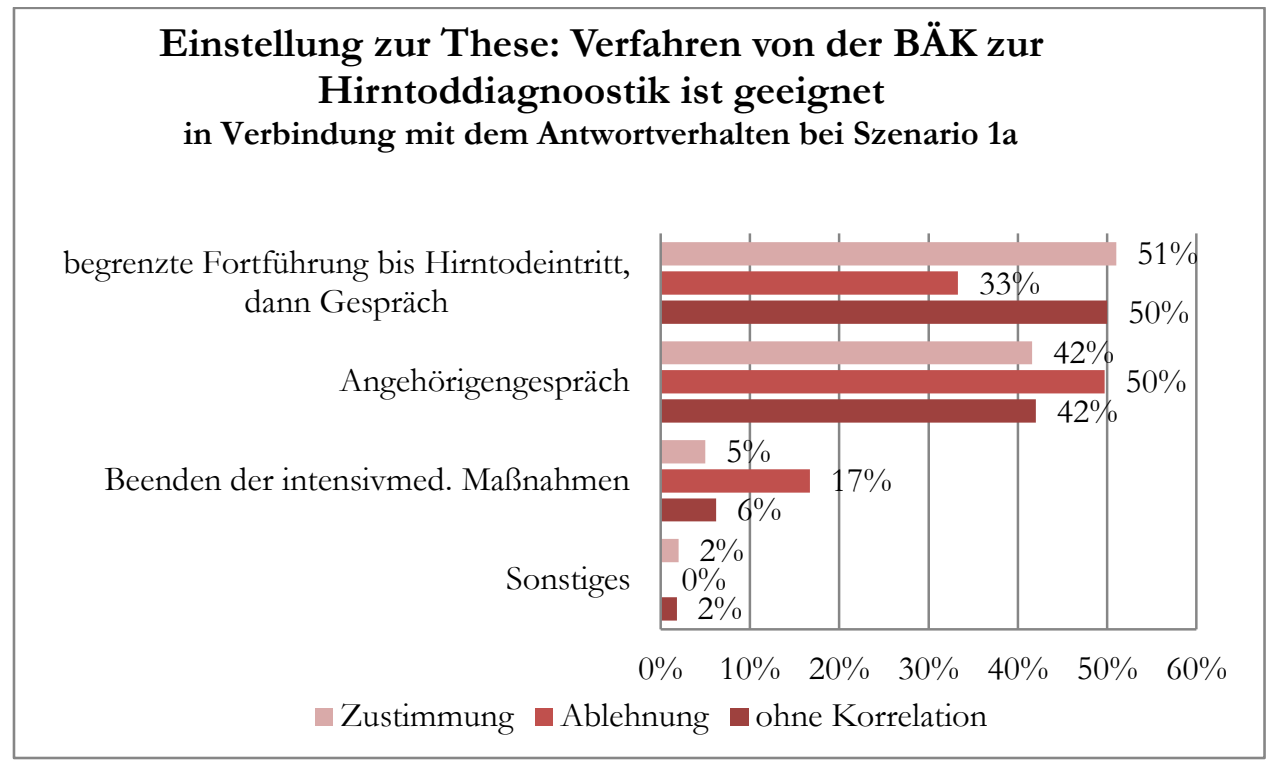

Das Beenden der intensivmedizinischen Maßnahmen wurde also unabhängig von der Einstellung der Teilnehmenden nie favorisiert und dem Angehörigengespräch oder dem Fortführen der intensivmedizinischen Maßnahmen vorgezogen - bei den Kritikern der Hirntoddiagnostik allerdings schon: Bei baldig eintretendem Hirntod und unbekannter Bereitschaft zur Organspende (Szenario 2b) votierten $42 \%$ der Kritiker gegen eine Organspende. Unter den Zustimmenden zur Hirntoddiagnostik wählten nur $27 \%$ bei Szenario 2b dieses Vorgehen, was dem Antwortverhalten der Befragten ohne Berücksichtigung der Einstellung entsprach (29\%). (Abb. 12)

Nicht nur bei einem baldig eintretenden Hirntod (Szenarien 2), sondern auch bei Szenario 1a (eingetretender Hirntod; dokumentierte Organspendebereitschaft) war auffällig, dass sich rund dreimal so viele der Kritiker des Verfahrens zur Hirntoddiagnostik (17\%) für das Beenden der intensivmedizinischen Maßnahmen entschieden, als Befürworter (5\%). Bei unbekannter Bereitschaft zur Organspende (Szenario 1b) entsprachen sich die Zahlen der Kritiker (17\%), der Befürworter (16\%) und die Zahlen unabhängig von der Einstellung zur Hirntoddiagnostik (18\%). 
Abbildung 12: Betrachtet wurde die Aussage, dass das Verfahren der BÄK zur Hirntoddiagnostik geeignet ist in Verbindung mit Szenario 2b (baldiger Hirntodeintritt/ unbekannte Organspendebereitschaft).

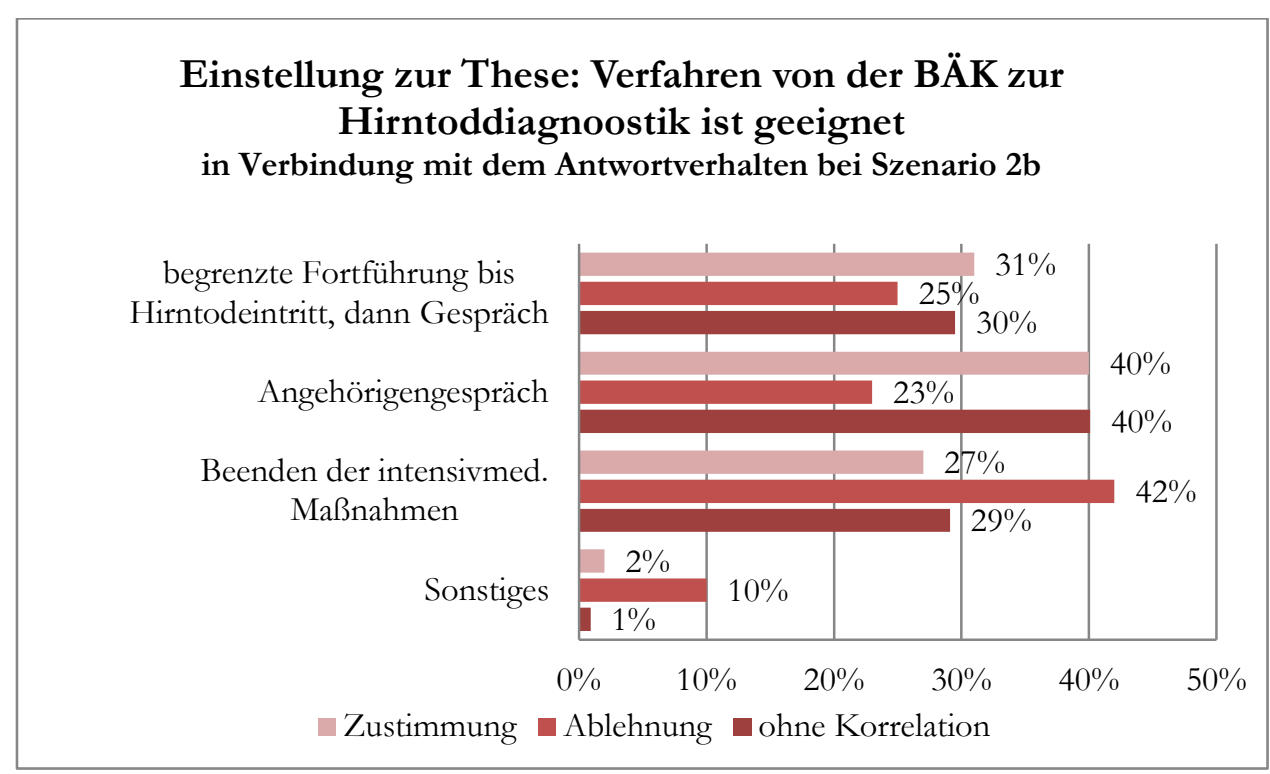

\subsubsection{Aussage: „Der Hirntod ist mit dem Tod des Menschen gleichzusetzen“}

Wie bereits bei der These zur Hirntoddiagnostik kam es hauptsächlich bei den Kritikern der Gleichsetzung des Hirntods mit dem Tod des Patienten zu Unterschiede verglichen mit den Daten ohne Berücksichtigung der Einstellung der Teilnehmenden.

Es sprachen sich wiederum mehr Befragte, die eine Gleichsetzung des Hirntods mit dem Tod des Menschen kritisch gesehen haben, gegen eine Organspende aus. Unabhängig vom Vorliegen eines Organspendeausweis sowie von den unterschiedlichen Szenarien. Besonders deutlich war diese Abweichung bei Szenario 2 (baldig eintretender Hirntod) zu beobachten: Fast ein Drittel (30 \%) der Kritiker des Hirntodkriteriums wählte bei Szenario 2a (dokumentierte Organspendebereitschaft) die Beendigung der Maßnahmen, bei unbekannter Bereitschaft zur Organspende waren es sogar $40 \%$. Ohne Berücksichtigung der Einstellung, also ohne Korrelation, sprachen sich bei vorliegendem Organspendeausweis (Szenario 2a) lediglich $14 \%$ der Teilnehmender für das Beenden der intensivmedizinischen Maßnahmen aus, bei Szenario 2b (unbekannte Organspendebereitschaft) $29 \%$.

Neben ihrer kritischen Einstellung zur Organspende fiel bei den Gegnern der Gleichsetzung zwischen Hirntod und Patiententod noch etwas auf: In Szenario 1a (vermuteter Hirntod; dokumentierte Organspendebereitschaft) entschied sich die Mehrheit der Kritiker (50\%) für ein Gespräch mit den Angehörigen, nur 30\% strebten die Fortführung der in- 
tensivmedizinischen Maßnahmen an. Ohne Korrelation, also ohne Berücksichtigung der Einstellung der Teilnehmenden, entschieden sich mehr Teilnehmende für die Fortführung (50\%) als für ein Gespräch mit den Angehörigen (42\%). (Abb. 13) Bei Szenario 2 (baldig eintretender Hirntod) wich das Antwortverhalten der Hirntodkritiker gänzlich von dem ohne Bezug auf die Einstellung ab. Unabhängig von der Einstellung votierten die Befragten bei bekannter Bereitschaft zur Organspende (Szenario 2a) zu gleichen Teilen für die Fortführung der intensivmedizinsichen Maßnahmen und für das Angehörigengespräch und favorisierten bei unbekannter Organspendebereitschaft (Szenario 2b) das Angehörigengespräch. Die Kritiker der Hirntoddiagnostik hingegen wählten bei Szenario 2a zwar mehrheitlich das Fortführen der Maßnahmen (35\%), sprachen sich aber ebenfalls zu jeweils knapp einem Drittel (30 \%) für das Gespräch mit den Angehörigen oder gegen eine Organspende aus. Bei Szenario 2b (baldig eintretender Hirntod; unbekannte Organspendebereitschaft) favorisierten die Teilnehmer sogar das Beenden der intensivmedizinsichen Maßnahmen (40\%), wie bereits die Kritiker der Hirntoddiagnostik bei derselben Konstellation. (Abb. 14)

Abbildung 13: Betrachtet wurde die Aussage, dass der Hirntod mit dem Tod des Menschen gleichzusetzen sei in Verbindung mit Szenario 1a (eingetretener Hirntod/ dokumentierte Organspendebereitschaft).

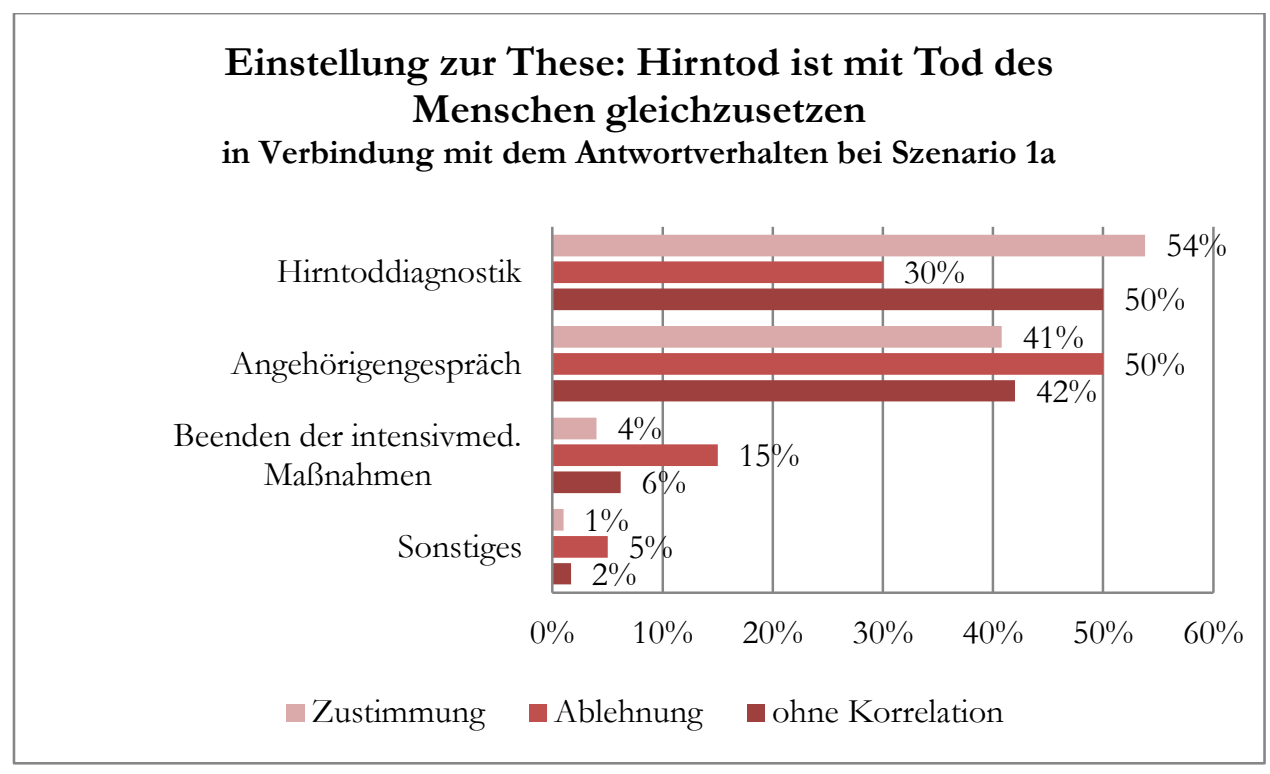


Abbildung 14: Betrachtet wurde die Aussage, dass der Hirntod mit dem Tod des Menschen gleichzusetzen sei in Verbindung mit Szenario $2 \mathrm{~b}$ (baldiger Hirntodeintritt/ unbekannte Organspendebereitschaft).

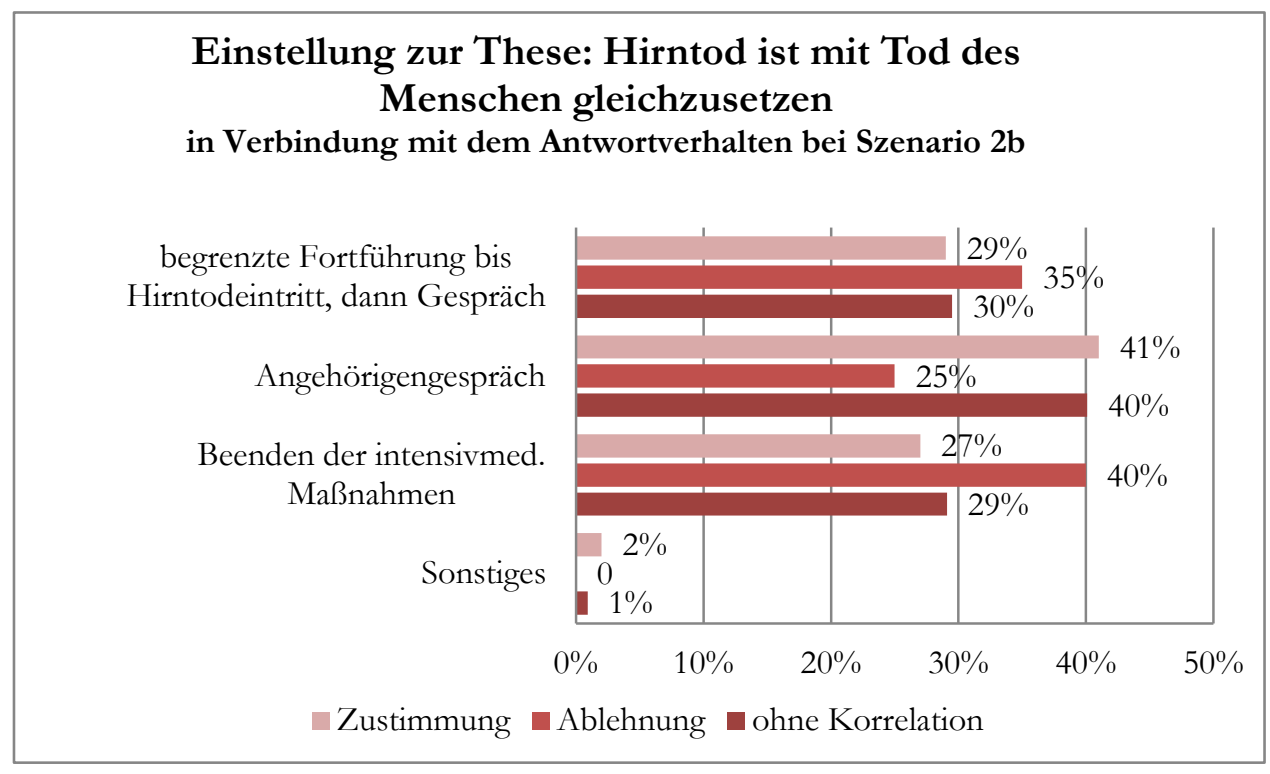

5.4.4.4 Aussage: „Hirntote Patienten sind erst dann wirklich tot, wenn das Herz nach Abschalten der Geräte zu schlagen aufgehört hat"“

Diese These, dass hirntote Patienten erst durch einen Herztod wirklich tot sind, steht im Widerspruch zu der vorangegangenen Aussage, dass der Hirntod dem Tod des Menschen entspreche, sowie auch zu dem gesamten Konzept der Hirntoddiagnostik (siehe Kapitel 5.3.1). Deshalb war es von besonderem Interesse, die Einstellung der Befragten bezüglich des Todes vom hirntoten Patienten mit dem Antwortverhalten bei den Szenarien in Verbindung zu setzen.

Zunächst war festzustellen, dass sich das Antwortverhalten im Bezug auf die These, dass der Tod des Menschen erst bei aufhörendem Herzschlag eintrete, kaum von dem ohne Berücksichtigung der Einstellung unterschied. Auch bei den Kritikern des Hirntodkonzepts und unabhängig von Zustimmung, Ablehnung oder den unterschiedlichen Szenarien. Allerdings gab es Diskrepanzen, wenn man die Antworten bezogen auf diese These mit dem Antwortverhalten der anderen Thesen verglich: Erstmals wurde das Vorgehen der Maßnahmenfortführung von den Kritikern der Gleichsetzung (Szenario 1a: 57 \%; Szenario 2a: $45 \%$; Szenario 2b: 34 \%), dass der Hirntod erst mit dem Herztod des hirntoten Patienten eintrete, häufiger gewählt als von denen, die eine Gleichsetzung befürworteten (Szenario 1a: $42 \%$; Szenario 2a: $38 \%$; Szenario 2b: $25 \%$ ). (Abb. 15) Bei den anderen Thesen hingegen votierten weniger Kritiker als Befürworter für die Fortführung der intensivmedizinischen Maßnahmen. 
Abbildung 15: Betrachtet wurde die Aussage, dass der Tod des Menschen erst bei aufhörendem Herzschlag auftrete, in Verbindung mit Szenario 1a (eingetretener Hirntod/ dokumentierte Organspendebereitschaft).

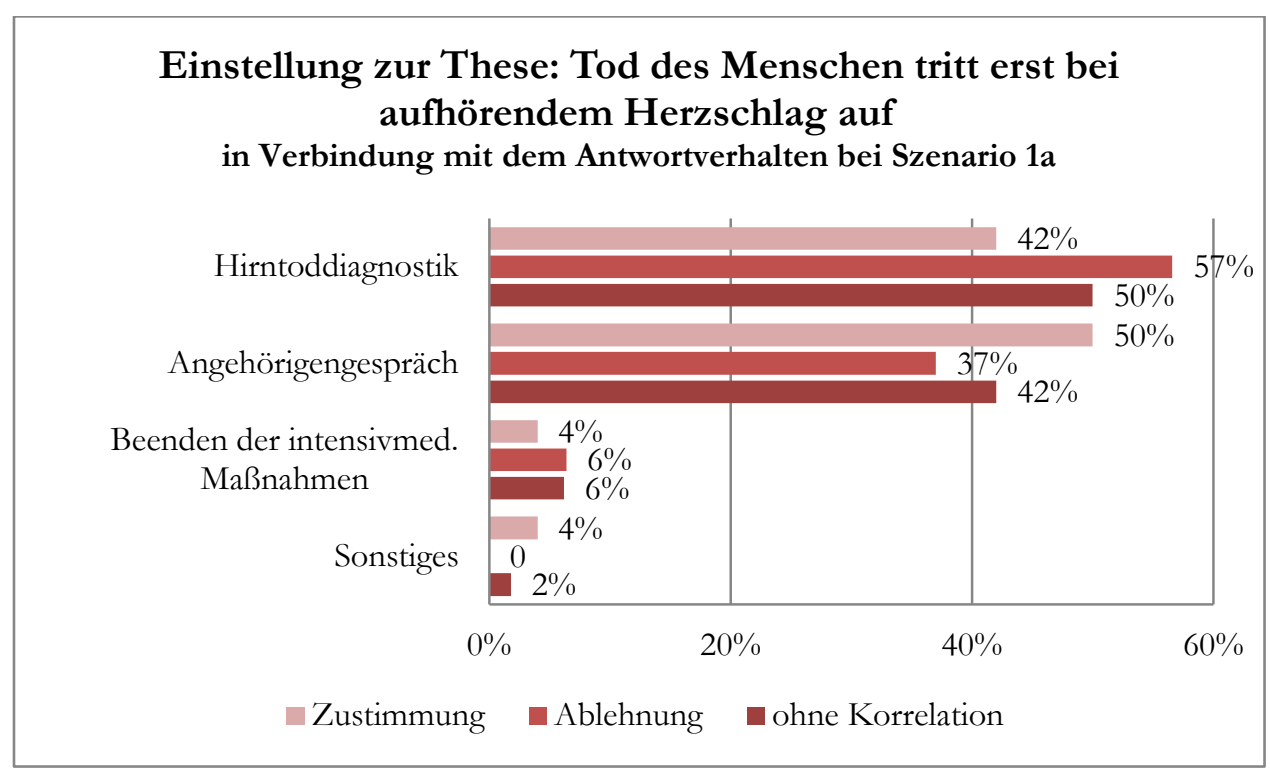

Ebenfalls bei Betrachtung des Antwortverhaltens gegen eine Organspende ergaben sich Auffälligkeiten verglichen zu den anderen Thesen: Bei Szenario 1b (vermuteter Hirntod; unklare Organspendebereitschaft) votierten etwas mehr der Befürworter der These für die Beendigung der intensivmedizinischen Maßnahmen (18\%), als bei Ablehnung dieser These (12\%). Hinsichtlich der anderen Thesen waren es sonst eher Kritiker, die sich vermehrt gegen eine Organspende aussprachen. Abgesehen davon gab es sonst keinerlei Signifikanzen hinsichtlich des Votums gegen eine Organspende, da die Zahlen mit den Daten unabhängig von der Einstellung zum Hirntodkonzept übereinstimmten.

Die Ergebnisse, dass eher die Kritiker der Gleichsetzung eine Fortführung der intensivmedizinischen Maßnahmen favorisierten und gleichzeitig die Befürworter bei Szenario 1b gegen eine Organspende votierten, sind nicht verwunderlich, da die Aussage, dass der Tod des Menschen erst bei aufhörendem Herzschlag eintrete, im Widerspruch zu den anderen Thesen steht: Da der Herztod nicht zu den von der BÄK festgelegten Todeskriterien gehört (siehe Kapitel 2.3.3.1), entspricht eine Befürwortung der These einer Kritik an dem Hirntodkonzept, eine Ablehnung allerdings befürwortet das Konzept der BÄK.

5.4.4.5 Aussage: „Patientenverfügungen sind keine verbindlichen Handlungsanweisungen, sondern geben einen Hinweis darauf, was der Patient gewollt haben könnte“

Die grundsätzliche Haltung der Befragten zur Patientenverfügung spielte für die Entscheidungen beim ersten Szenario (vermuteter Hirntod) kaum eine Rolle. Es fiel aber auf, dass 
sich das Antwortverhalten im zweiten Szenario (baldig eintretender Hirntod) zwischen denen, die die Patientenverfügung als verbindlich ansahen, und denen, die sie als unverbindlich einschätzen, unterschied: In Szenario 2 (baldig eintretender Hirntod) sprachen sich die Befragten, die eine Patientenverfügung als verbindlich empfanden, eher für die begrenzte Fortführung der intensivmedizinischen Maßnahmen bis zum Hirntodeintritt aus (Szenario 2a: $44 \%$; Szenario 2b: $34 \%$ ) als für das Angehörigengespräch (Szenario 2a: 37 \%; Szenario 2b: 30 \%). Sowohl bei den Befragten, die die Patientenverfügung als unverbindlich sahen, als auch bei den Daten ohne Verbindung zur Einstellung bezüglich der Verfügung war die Gewichtung genau umgekehrt: Die Befragten favorisierten sowohl bei Szenario 2a (Zustimmende: $48 \%$; ohne Korrelation: $43 \%$ ) als auch bei Szenario 2b (Zustimmende: 48 \%; ohne Korrelation: $40 \%$ ) eher das Gespräch mit den Angehörigen als die intensivmedizinische Fortführung der Maßnahmen (Szenario 2a: Zustimmende: 38 \%; ohne Korrelation: 41 \%/ Szenario 2b: Zustimmende: $25 \%$; ohne Korrelation: $30 \%$ ). (Abb. 16)

Bei Betrachtung des Votums gegen eine Organspende gab es nur eine geringe Abweichungen zu den Daten ohne Verbindung mit der Einstellung zur Patientenverfügung: Knapp ein Drittel (34\%) derer, die die Patientenverfügung als verbindlich ansahen, wählte bei Szenario 2b (baldiger Hirntodeintritt; unbekannte Organspendebereitschaft) das Beenden der intensivmedizinischen Maßnahmen. Von denen, die die Verfügung als unverbindlich empfanden, sowie bei den Daten ohne Korrelation war es bei der Konstellation des zweiten Szenarios ein geringerer Anteil (Zustimmende: $25 \%$; ohne Korrelation: $29 \%$ ). (Abb. 16) 
Abbildung 16: Betrachtet wurde die Aussage, dass die Patientenverfügung keine verbindliche Handlungsanweisung sei, in Verbindung mit Szenario 2b (baldiger Hirntodeintritt/ unbekannte Organspendebereitschaft).

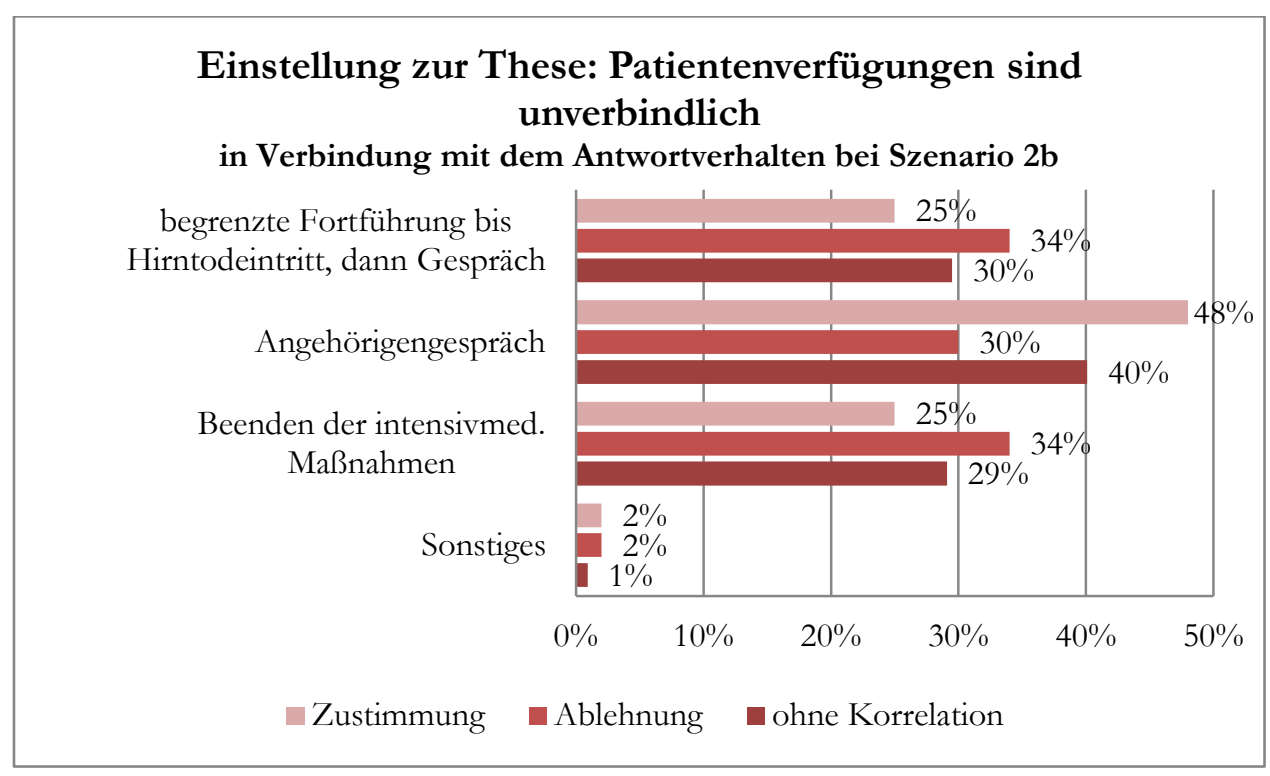

5.4.4.6 Zusammenfassung der Korrelation zwischen den Standpunkten und Handlungsentscheidungen

Insgesamt wurde deutlich, dass sich das Antwortverhalten der Befragten insoweit kaum von den Ergebnissen bei Berücksichtigung der jeweiligen grundsätzlichen Einstellung der Befragten zu den Themen Organspendetransplantation, Hirntoddiagnostik und Patientenverfügung unterschied, als sich auch bei Berücksichtigung der jeweiligen Grundeinstellung die Mehrheit der Befragten für die Möglichkeit einer Organspende entschied.

Allerdings fiel auf, dass das Antwortverhalten verbunden mit der Einstellung größtenteils dann von dem ohne Berücksichtigung der Einstellung abwich, sobald die Themen kritisch beurteilt wurden: Einerseits dahingehend, dass die Kritiker der Organtransplantation, der Hirntoddiagnostik und des Hirntodkonzepts sich zurückhaltender als die Befürworter der jeweiligen Thesen für die Fortführung der intensivmedizinischen Maßnahmen aussprachen. Andererseits votierten dann auch mehr der Kritiker für das Beenden der intensivmedizinischen Maßnahmen, vor allem bei Szenario 2b, wo die Bereitschaft zur Organspende fraglich war und der Eintritt des Hirntods des Patienten vermutlich baldig eintreten sollte. Sowohl die Gegner der Hirntoddiagnostik als auch diejenigen, die den Hirntod nicht als Tod des Menschen sahen, favorisierten in dieser Konstellation sogar das Beenden der intensivmedizinischen Maßnahmen. 
Allerdings gestaltete es sich bei zwei Thesen, die einerseits dem Hirntodkonzept widersprachen und andererseits die Verbindlichkeit der Patientenverfügung beurteilten, anders: Sowohl bei denen, die den Hirntod nicht als Herztod des hirntoten Patienten gleichsetzten (also das Hirntodkriterium befürworteten), als auch bei denen, die die Patientenverfügung als verbindlich einstuften, entschieden sich jeweils bei vorliegendem Organspendeausweis (Szenario 1a und 2a) mehr für eine Fortführung der intensivmedizinischen Maßnahmen als die Gegner des Konzepts bzw. als diejenigen, die eine Patientenverfügung als unverbindlich einstuften, und auch als die Befragten, die das Angehörigengespräch wählten. Der Anteil derer, die für das Beenden der intensivmedizinischen Maßnahmen votierten, war bei diesen beiden Thesen geringer als im Zusammenhang mit den anderen Thesen, vor allem bei Szenario 2b (baldig eintretender Hirntod; unbekannte Organspendebereitschaft). Wobei auch da der Anteil der Befürworter des Hirntodkonzepts bzw. derer die eine Patientenverfügung verbindlich empfanden, die sich gegen eine Organspende entschieden, höher war, als unter denen die eine entgegengesetzte Meinung hatten, jedoch war der Abstand nicht so hoch.

\subsection{Einfluss der soziodemographischen Daten auf das Antwort- verhalten}

Alter, Geschlecht und Religionszugehörigkeit der Befragten hatten keinen signifikanten Einfluss auf das Antwortverhalten der Befragten.

\subsection{Unterstützung bei der klinischen Entscheidungsfindung}

Dieser Teil des Fragebogens beschäftigte sich mit der Form der Unterstützung, die sich die Befragten im Falle konfligierender Aussagen in der Patientenverfügung und der Organspendeerklärung wünschen und mit dem Ausmaß der Unterstützung, die die ärztlichen und pflegenden Mitarbeitenden in der Verfahrensempfehlung der UMG sehen.

\subsubsection{Favorisierte Unterstützung}

$92 \%$ der Befragten wünschten sich eine Unterstützung bei der klinischen Entscheidungsfindung. Die große Mehrheit forderte kompetente Ansprechpartner, die entweder zu einem Angehörigengespräch (80\%) oder in konflikthaften Situationen beratend bzw. moderierend hinzugezogen werden können (62\%). Darüber hinaus wünschten sich die Befragten Unterstützung in Form ethischer Handlungsempfehlungen (60\%) sowie in Form von Informationen zum rechtlichen Hintergrund (44\%). (Abb. 16) 
Beim Vergleich der beiden Professionen fiel auf, dass sich die Pflegenden vor allem einen kompetenten Ansprechpartner beim Gespräch mit den Angehörigen (85 \%) und in geringerem Maße einen Berater bzw. Moderator für konflikthafte Situationen (60 \%) wünschten. Die ärztlichen Mitarbeitenden hingegen wünschten sich beide Ansprechpartner in nahezu gleichem Maße (63\% für das Angehörigengespräch; $67 \%$ für die Beratung bzw. Moderation in konflikthaften Situationen). Ferner fällt auf, dass über die Hälfte der ärztlichen Mitarbeitenden (52\%) allgemeine Informationen zum rechtlichen Hintergrund forderten, während die Pflegenden mit $41 \%$ dies in geringerem Maße taten.(Abb. 17)

Nur $4 \%$ der Teilnehmenden wünschte sich keine Unterstützung bei der klinischen Entscheidungsfindung (Ärztinnen und Ärzte: 7 \%; Pflegende: $3 \%$ ).

Abbildung 17: Gewünschte Unterstützung für die Szenarien bei der klinischen Entscheidungsfindung (Mehrfachantwort möglich).

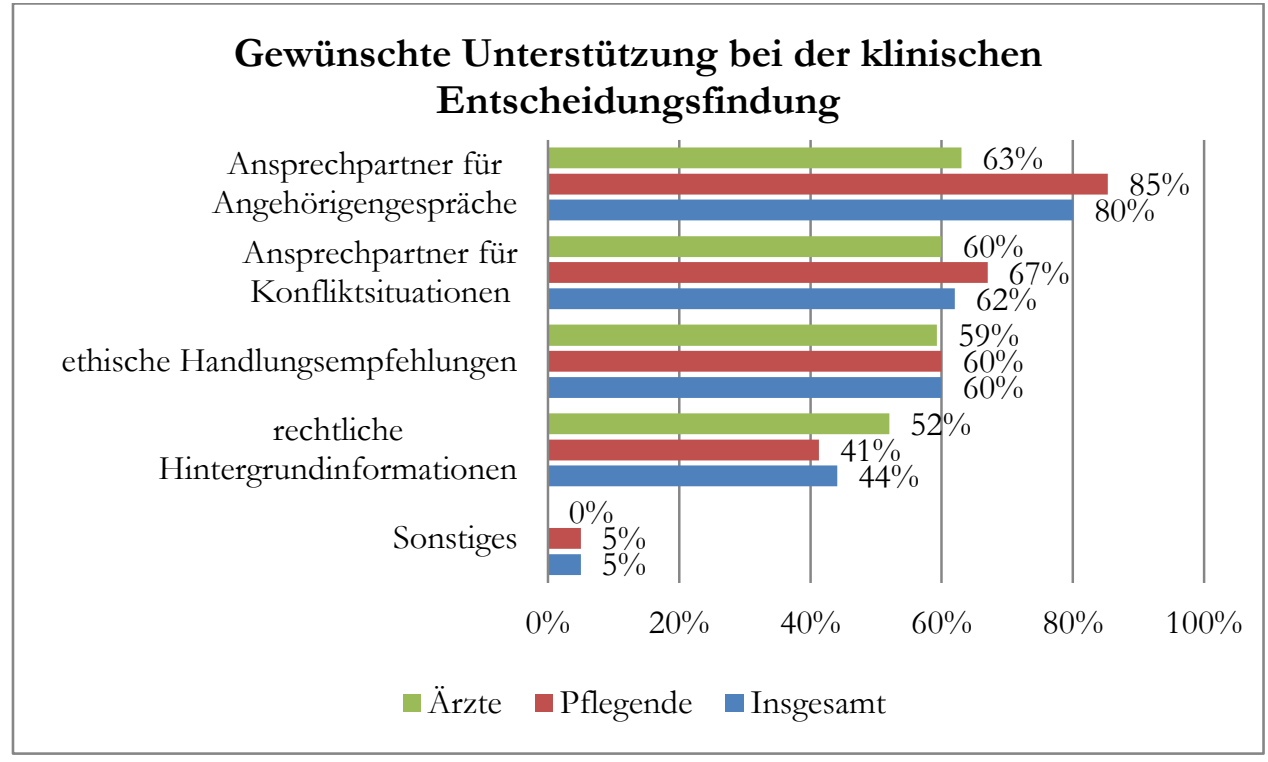

\subsubsection{Verfahrensempfehlung der UMG als Unterstützung}

Zwei Drittel der Befragten (66\%) gaben an, die Verfahrensempfehlung der UMG zum Thema Patientenverfügung und Organspendeerklärung (siehe Kapitel 2.4.2) nicht zu kennen. $29 \%$ hatten von dieser Empfehlung zwar gehört, hatten sie aber entweder nicht gelesen oder konnten sich an deren Inhalt nicht erinnern. Nur $5 \%$ der Teilnehmenden gaben an, den Inhalt der Empfehlung zu kennen. (Abb. 18)

Da es für die Bewertung der Verfahrensempfehlung der UMG von entscheidender Bedeutung ist, dass man deren Inhalt kennt, wurden im Folgenden nur die Teilnehmenden berücksichtigt, die den Inhalt der Empfehlung kannten. Von diesen $5 \%$ empfanden alle die Verfahrensempfehlung als hilfreich. Rund ein Drittel (33\%) sogar als „sehr hilfreich“. 
Zwischen den Professionen gab es lediglich den Unterschied, dass von den Ärztinnen und Ärzten keiner die Verfahrensempfehlung als „sehr hilfreich“ einschätzte. Von den pflegenden Mitarbeitenden waren es $40 \%$.

Abbildung 18: Bekanntheitsgrad der Verfahrensempfehlung der UMG unter den Befragten.

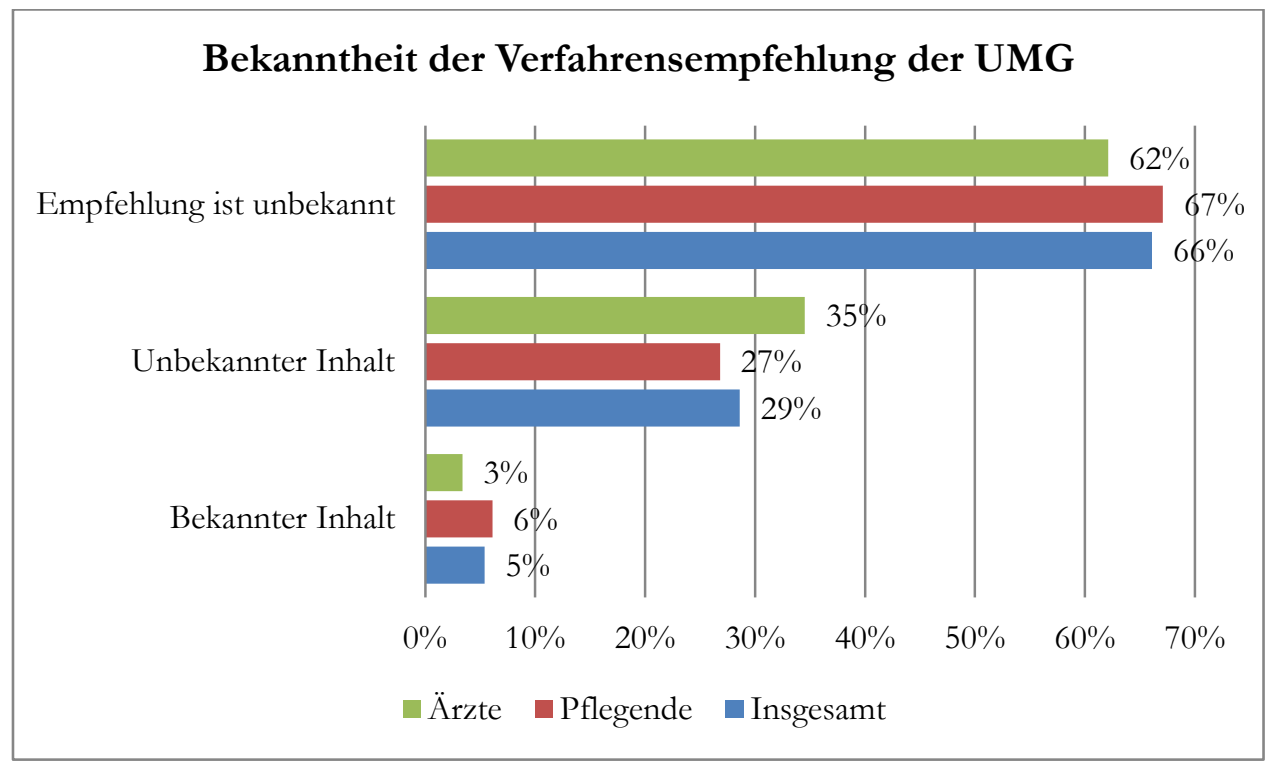

\subsubsection{Zusammenfassung zur Unterstützung bei der klinischen Entscheidungs- findung}

Generell wird also deutlich, dass sich die Mehrheit der Befragten eine Unterstützung bei der klinischen Entscheidungsfindung wünscht. Die Teilnehmenden forderten Ansprechpartner, die in konflikthaften Situationen beratend bzw. moderierend oder zu Angehörigengesprächen hinzugezogen werden könnten. Eine andere vorstellbare Form der Unterstützung stellte eine ethische Handlungsempfehlung für die Befragten dar. Die bereits vorhandene Verfahrensempfehlung der UMG war bei den Befragten größtenteils unbekannt. Diejenigen, die den Inhalt der Empfehlung kannten, stuften sie sämtlich als hilfreich ein. 


\section{Diskussion}

Die Aktualität des Konflikts zwischen der Patientenverfügung und dem Organspendeausweis verdeutlichen die Veröffentlichungen der BÄK sowie der UMG. Ein Novum in der Debatte stellt die hier durchgeführte Befragung seitens der Pflegenden und Ärztinnen und Ärzten an der UMG zu diesem Konflikt dar. Erstmals wird die Perspektive der Anwender und deren Einschätzung beleuchtet. Mithilfe dieser Studie sollte ein Stimmungsbild gezeichnet werden, das die Diskussion über die Probleme im Umgang mit den Dokumenten bereichern soll.

Im Folgenden werden die Studienergebnisse diskutiert und mögliche Konsequenzen für die Debatte über den Konflikt zwischen Patientenverfügung und Organspendeausweis aufgezeigt werden.

\subsection{Bewertung des Konflikts zwischen Patientenverfügung und Organspendeerklärung}

Durch die Studie wurde deutlich, dass die Pflegenden stärker als die Ärztinnen und Ärzte dazu neigen, die Bereitschaft des Patienten zur Organspende nicht zu beachten. Warum sprachen sich die Pflegenden oft gegen eine Organspende aus?

Um diese Frage beantworten zu können, wird zunächst der Unterschied zwischen den beiden Professionen hinsichtlich des Antwortverhaltens beleuchtet. Anschließend werden die Ergebnisse in Zusammenhang mit den persönlichen Einstellungen der handelnden Personen zu den Themen Hirntod, Organspende und Patientenverfügung gebracht.

\subsubsection{Antwortverhalten der Professionen hinsichtlich der unterschiedlichen Szenarien}

Ein vergleichsweise hoher Anteil der pflegenden Mitarbeiter sprach sich trotz des Vorliegens eines Organspendeausweises (Szenarien 1a und 2a) für eine Beendigung der intensivmedizinischen Maßnahmen und somit gegen eine möglich Organspende aus. Sowohl im Falle des vermuteten, als auch im Falle des erwarteten Hirntodes (Szenario 1a: 8 \%; Szenario 2a: $19 \%$ ). Von den Ärztinnen und Ärzten hingegen sprach sich bei vorliegendem Organspendeausweis (Szenarien 1a und 2a) niemand für das Beenden der lebenserhaltenden Maßnahmen aus. Im Falle einer nicht dokumentierten Bereitschaft zur Organspende (Szenarien 1b und 2b) gab es zwar einige Ärztinnen und Ärzte, die sich für das Beenden 
der lebenserhaltenden Maßnahmen aussprachen, ihr Anteil war mit 10\% (Szenario 1b) bzw. $17 \%$ (Szenario 2b) aber deutlich niedriger als bei den Pflegenden (Szenario 1b: 21 \%; Szenario 2b: $31 \%$ ).

Einige Pflegende ziehen also bei Vorliegen einer entsprechenden Patientenverfügung die Möglichkeit einer Organspende gar nicht in Betracht, obwohl diese dem erklärten bzw. dem (potentiell) mutmaßlichen Willen des Patienten entspricht. Sie räumen dem in der Patientenverfügung dokumentierten Wunsch nach Therapiebegrenzung ein höheres Gewicht ein, als der im Organspendeausweis dokumentierten Bereitschaft zur Organspende. Da aber beide Dokumente dem Willen des Patienten entsprechen, müssen eigentlich sowohl die Patientenverfügung als auch der Organspendeausweis berücksichtigt werden (siehe Kapitel 2.4).

Im Gegensatz dazu ist zu erkennen, dass die ärztlichen Mitarbeiter die Möglichkeit der Organspende stärker im Blick haben. So sprachen sich bei Vorliegen eines entsprechenden Organspendeausweises $66 \%$ (Szenario 1a) bzw. $57 \%$ (Szenario 2a) der Ärztinnen und Ärzte für eine Fortführung der intensivmedizinischen Maßnahmen zum Zwecke der Realisierung einer möglichen Organspende und weitere $35 \%$ (Szenario 1a) bzw. $43 \%$ (Szenario 2a) für die Klärung des weiteren Vorgehens einschließlich der Erörterung der Möglichkeit einer Organspende mit den Angehörigen der Patienten aus. Bei nicht dokumentierter Bereitschaft zur Organspende favorisierten die ärztlichen Mitarbeiter in erster Linie das Angehörigengespräch (Szenario 1b: 52\%; Szenario 2b: 53\%) und zweitrangig das initiale Fortführen der intensivmedizinischen Maßnahmen (Szenario 1b und 2b: $31 \%$ ). Für die Ärztinnen und Ärzte scheint also im Konflikt zwischen der Patientenverfügung und dem Organspendeausweis die Entscheidung eher zu Gunsten der Organspende zu fallen. Sobald die Breitschaft zur Organspende unklar war, wurde trotz einer vorliegenden Patientenverfügung, in der sich der Patient gegen lebenserhaltende Maßnahmen aussprach, die Möglichkeit einer Organspende nicht übergangen, sondern im Gespräch mit den Angehörigen diskutiert.

Auch die Pflegenden entschieden sich zu großen Teilen für das Angehörigengespräch, allerdings nicht nur bei unbekannter Bereitschaft zur Organspende: Am deutlichsten in Szenario 1a und b (vermuteter Hirntod; dokumentierte Organspendebereitschaft: $45 \%$; unbekannte Organspendebereitschaft: $53 \%$ ) und in Szenario 2a (baldig eintretender Hirntod; dokumentierte Organspendebereitschaft: 43 \%). Außer in Szenario 2b, bei dem zweitrangig das Beenden der intensivmedizinischen Maßnahmen gewählt wurde (31\%), votierten die Pflegenden in zweiter Linie oder zu gleichen Teilen (Szenario 1a) für das Fortführen der 
intensivmedizinischen Maßnahmen (Szenario 1a: 45\%; Szenario 1b: $27 \%$; Szenario 2a: $35 \%)$.

Das Angehörigengespräch scheint dementsprechend für die Ärztinnen und Ärzte und auch für die Pflegenden eine große Rolle zu spielen. Daraus kann man den Schluss ziehen, dass sowohl die ärztlichen als auch die pflegenden Mitarbeiter die Kenntnis der Angehörigen um den mutmaßlichen Patientenwillen voraussetzen und für ihre eigene Entscheidung als mit ausschlaggebend einschätzen. Vor allem im Zwiespalt zwischen zwei sich scheinbar widersprechenden Willensäußerungen ist das Angehörigengespräch sicherlich ein guter Weg. Trotzdem muss man dabei berücksichtigen, dass es auch für die Angehörigen schwierig sein kann, den mutmaßlichen Willen des Patienten richtig wiederzugeben. Mehr noch, es kann sogar mangels richtiger Einschätzung zu aus Sicht des Patienten unerwünschten Therapieentscheidungen und damit zu Fremdbestimmungen kommen (Marckmann und in der Schmitten 2013). Daneben stellt die Aufgabe, den mutmaßlichen Willen des Patienten zu bestimmen, für manche Angehörige auch eine besondere Belastung dar (Jox 2012). Die Deutsche Alzheimer Gesellschaft fasst dazu zusammen:

„Festlegungen [in einer Patientenverfügung] getroffen zu baben, kann für Patienten im Voraus eine Berubigung und für ibre Angebörigen später eine Entlastung bedeuten. "

\subsubsection{Allgemeine Einstellung zu Organspende, Hirntod und Patientenverfügung}

Wie bereits im vorangegangenen Kapitel beschrieben zeigen die Ergebnisse der Studie, dass die pflegenden Mitarbeiter der befragten Intensivstationen der Organspende gegenüber kritischer eingestellt sind, als die ärztlichen Mitarbeitenden (siehe Kapitel 5.4.3).

Was könnten mögliche Gründe für die kritische Haltung der Pflegenden sein? Inwiefern werden die im vorangegangenen Abschnitt (siehe Kapitel 6.1.1) dargelegten Ergebnisse von Unterschieden in der Einstellung zu Organspende, Hirntod und Patientenverfügung zwischen den Professionen beeinflusst?

Zur Beantwortung dieser Fragen wurde die Einstellungen der Teilnehmenden hinsichtlich der Organspende und der Hirntoddiagnostik analysiert. Dabei spielte auch die grundsätzliche Einstellung gegenüber der Patientenverfügung und gegenüber dem Organspendeausweis eine Rolle, da gerade im Konflikt zwischen der Patientenverfügung

\footnotetext{
${ }^{4}$ Deutsche Alzheimer-Gesellschaft e.V. (Hrsg.) (2012): Empfehlungen zum Umgang mit Patientenverfügungen bei Demenz. https://www.alzheimer-bw.de/fileadmin/AGBW Medien/Dokumente/ Empfehlungen DAlzG/empfehlungen-Patientenverfuegung-20120213 01.pdf. (Datum des Zugriffs: 04.10.2016)
} 
einerseits und dem Organspendeausweis andererseits die Bewertung der einzelnen Dokumente die Entscheidung für oder gegen eine Organspende beeinflusst.

\subsubsection{Einstellung zur Organtransplantation und Hirntoddiagnostik}

Schon die Antworten auf die erste Frage des Fragebogens machen deutlich, dass die Einstellung gegenüber den beiden Dokumenten bei den zwei Berufsgruppen unterschiedlich ist. Zwar nannten pflegende und ärztliche Mitarbeiter die bekannt gewordenen Skandale um Organspenden sowie eine mangelnde Aufklärung der Öffentlichkeit als Hauptgründe für den Rückgang der postmortalen Organspende. Auffällig war aber, dass die Ärztinnen und Ärzte darüber hinaus vor allem organisatorische Barrieren, die Pflegenden eher grundsätzliche Bedenken nannten. Die ärztlichen Mitarbeiter führten als weitere Gründe für den Rückgang der Organspenden fehlende Informationen bezüglich gesetzlicher Regelungen, mangelnde Kooperation zwischen Spenderkrankenhäusern und Transplantationszentren sowie eine mangelnde finanzielle Entschädigung der Spenderkrankenhäuser an. Die weiteren Gründe der Pflegenden bezogen sich vor allem auf die Hirntoddiagnostik: Zweifel an der Sicherheit der Hirntoddiagnostik sowie ethische Bedenken gegenüber dem Hirntodkriterium wurden genannt.

\subsection{Zeugt die kritische Einstellung der Pflegenden also von einem Misstrauen gegenüber der Hirntoddiagnostik?}

Bei der Frage nach der Eignung des von der BÄK vorgesehenen Verfahrens zur Hirntoddiagnostik stimmten die pflegenden Mitarbeiter deutlich der Aussage zu, dass das Verfahren geeignet sei, den irreversiblen Ausfall aller Hirnfunktionen nachzuweisen (88 \%). Zwar etwas zurückhaltender als die Ärztinnen und Ärzte (97\%), aber trotzdem deutlich. Das verbleibende Misstrauen lässt sich eventuell durch die Festlegung des Todeskriteriums begründen. So war fast die Hälfte der Pflegenden (47\%) davon überzeugt, dass bereits der Hirntod als Tod des Menschen anzusehen sei. Ein nahezu ebenso großer Anteil (43\%) war jedoch der Auffassung, dass der Tod des Menschen bei hirntoten Patienten erst durch einen Herzstillstand eintrete.

Die Pflegenden sahen also einerseits die Diagnostik als hinreichend sicher an, waren aber andrerseits der Meinung, dass der Hirntod kein angemessenes Kriterium darstellt, den Tod des Menschen festzustellen, weil das, was den Tod des Menschen ausmacht, nicht allein der irreversible Verlust seiner Hirnfunktionen ist. Sind die Pflegenden der Organspende gegenüber also kritischer eingestellt, weil sie den Herztod als relevant einschätzen? Zur Beantwortung dieser Frage wurde im Vergleich das Antwortverhalten der Ärztinnen und Ärzten 
dazu näher betrachtet. Dabei stellte sich heraus, dass sie dieselbe Einstellung hatten wie die Pflegenden. So hielten die ärztlichen Mitarbeitenden, jeweils in etwas höherem Anteil als die Pflegenden, die Hirntoddiagnostik für geeignet (Ärztinnen und Ärzte: 97 \%; Pflegende: $88 \%$ ) und den Hirntod für den Tod des Menschen (Ärztinnen und Ärzte: 53 \%; Pflegende: $47 \%$ ). Fast genauso viele Ärztinnen und Ärzte (44\%) wie auch Pflegende (43\%) glaubten aber, dass der Tod mit dem Herzschlag zu assoziieren sei.

Dementsprechend sind die Pflegenden der Hirntoddiagnostik gegenüber zwar etwas kritischer eingestellt als die ärztlichen Kollegen, aber in so geringem Ausmaß, dass sich das unterschiedliche Antwortverhalten in den Szenarien dadurch nicht begründen lässt.

6.1.2.1.2 Beruht die kritische Einstellung gegenüber der Hirntoddiagnostik auf mangelnder Erfahrung der Pflegenden mit dem Thema Organspende?

Das Gegenteil scheint der Fall zu sein: Die Pflegenden verfügten im Schnitt eher über mehr Erfahrung mit der Hirntoddiagnostik und der Betreuung von Organspendern als das ärztliche Personal (Kapitel 5.2.2). Dies deutet darauf hin, dass mit zunehmender Erfahrung die Einstellung zu dem Thema Hirntod und Organspende kritischer wird.

Diese Beobachtung wurde auch in einer vergleichbaren Studie zur „Einstellung von potenziell am Organspendeprozess beteiligten Ärzten und Pflegekräften in Bayern“ gemacht. Diese ergab, dass die ärztlichen und pflegenden Mitarbeitender von Transplantationszentren die Organspende im Durchschnitt weniger positiv bewerteten als die Mitarbeitenden anderer Krankenhäuser (Ärztinnen und Ärzte: 86\% / 90 \%; Pflegende: 68\% / 78 \%) (Grammenos et al. 2014).

\subsection{Hegen die Pflegenden eine generelle Abneigung gegen die Organspende?}

Trotz der (im Vergleich zu den Ärztinnen und Ärzten) kritisch(er)en Haltung der Pflegenden kann man nicht von einer generellen Ablehnung der Organspende sprechen: Ein Großteil der Pflegenden (88 \%) sowie ein noch größerer Anteil der Ärztinnen und Ärzten (97\%) bejahte die Aussage, wonach die Organspende eine Hilfe für Schwerkranke darstellt. Außerdem verfügte fast die Hälfte der Pflegenden (42\%) über einen Organspendeausweis. Bei einer generellen Ablehnung gegen das Thema der Organspende wäre das nicht zu erwarten.

\subsubsection{Einstellung zur Patientenverfügung}

Auch bei der Einstellung zum Instrument der Patientenverfügung gab es Unterschiede zwischen den pflegenden und ärztlichen Mitarbeitenden. Über die Hälfte (55\%) der ärztli- 
chen Mitarbeiter stimmte der Aussage zu, wonach Patientenverfügungen keine verbindlichen Handlungsanweisungen sind, sondern nur einen Hinweis darauf geben, was der Patient gewollt haben könnte. Diese Einschätzung, die eigentlich dem 2009 beschlossenen Gesetz zur Patientenverfügung ( $($ 1901a Abs. 1 BGB) widerspricht, kann man als Ausdruck von Unsicherheit, aber auch als Ergebnis der Erfahrung sehen, dass viele der von Patienten erstellten Patientenverfügungen nicht ausreichend konkret und situationsbezogen sind, sodass daraus nicht unmittelbar ein bindender Patientenwille abgeleitet werden kann (BGH Urteil vom 06.07.2016 Az. XII ZB 61/16). Für letzteres spricht auch die Tatsache, dass die Ärztinnen und Ärzte mehrheitlich der Meinung waren, dass die Patientenverfügung im klinischen Alltag stärkere Berücksichtigung findet (79\%).

Genau anders herum haben es die Pflegenden gesehen: Sie betrachteten die Patientenverfügung als ein verbindliches Dokument (57\%), das aber in der Praxis keine ausreichende Berücksichtigung finde (46\%). Die Einschätzung der Pflegenden, wonach Patientenverfügungen vom medizinischen Personal oft nicht beachtet werden, wurde bereits in der Vergangenheit in der Forschung beobachtet. Demnach wird häufig die Entscheidung des Patientenvertreters (Betreuer oder Bevollmächtigter) bzw. die der Angehörigen der Patientenverfügung vorgezogen (Evans et al. 2012). Die Pflegenden haben also den Eindruck, dass die Patientenverfügungen von den Ärztinnen und Ärzten nicht ausreichend beachtet werden, was aber nicht bedeuten muss, dass der ärztliche Umgang damit nicht angemessen ist. Es könnte auch ein Hinweis darauf sein, dass Pflegende Patientenverfügungen vorschnell anwenden wollen. In jedem Fall ist es aber ein Hinweis auf eine mangelnde Transparenz der ärztlichen Entscheidung. Da nach Einschätzungen der Pflegenden sowie auch der Ärztinnen und Ärzte die Anzahl der Patientenverfügungen im Laufe der letzten Jahre merklich zugenommen hat, sollte der Umgang mit dem Dokument jedoch klar sein.

6.1.2.2.1 Lässt sich das kritische Verhalten der Pflegenden gegenüber einer Organspende durch ihre Einstellung gegenüber der Patientenverfügung erklären?

Deutlich wird, dass die Pflegenden, eher als die Ärztinnen und Ärzte, die Patientenverfügung als verbindlich ansehen, aber eine geringere Berücksichtigung seitens der Ärztinnen und Ärzte im klinischen Alltag feststellten. Im Konflikt zwischen den beiden Dokumenten, dem Organspendeausweis einerseits und der Patientenverfügung andererseits, hat das dazu geführt, dass die Pflegenden sich eher für eine Patientenverfügung und damit gegen eine mögliche Organspende entschieden haben. Die Pflegenden neigen also dazu, dem in der Patientenverfügung dokumentierten Wunsch nach Therapiebegrenzung ein höheres Gewicht einzuräumen als der im Organspendeausweis dokumentierten Bereitschaft zur Or- 
ganspende. Die ärztlichen Mitarbeiter andererseits, die die Patientenverfügung als unverbindlich einstuften, sprachen sich eher für eine Organspende aus (Kapitel 6.1.1). In Ergänzung zur vorliegenden Patientenverfügung war ihnen auch das Angehörigengespräch wichtig, um den Willen des Patienten herauszufinden. Dieses Verhalten verdeutlicht die kritische Haltung der ärztlichen Mitarbeiter gegenüber der Patientenverfügung.

Somit lässt sich die Einschätzung der Patientenverfügung mit den Entscheidungen in den Szenarien in Einklang bringen: Die Pflegenden, die der Patientenverfügung einen hohen Stellenwert anrechneten, entschieden sich oft der Verfügung gemäß und vernachlässigten den Organspendeausweis bzw. die Möglichkeit einer Organspende. Die Ärztinnen und Ärzte, die die Patientenverfügung als unverbindlich empfanden, arbeiten im klinischen Alltag mit anderen Willensbekundungen: Sei es der Organspendeausweis oder der im Gespräch mit den Angehörigen ermittelte mutmaßliche Patientenwille.

\subsubsection{Bewertung der Korrelation zwischen den Szenarien}

Die Rolle der Einstellung der Befragten hinsichtlich der Themen Organspende, Hirntod und Patientenverfügung wurde ebenfalls deutlich, wenn man diese Einstellung in Korrelation zu dem gewählten Vorgehen in den Szenarien setzte. Demzufolge entschieden sich diejenigen, die den Thesen kritisch gegenüberstanden, eher gegen eine Organspende, unabhängig vom Inhalt der vorliegenden Dokumente (siehe Kapitel 1.4.4).

\subsubsection{Die Rolle der Patientenverfügung in Korrelation zu den Szenarien}

Von besonderem Interesse war es dabei, auf die Rolle der Patientenverfügung einzugehen, da die Einschätzung des Dokuments, wie im vorangegangenen Abschnitt beschrieben, für das favorisierte Vorgehen eine Rolle spielt.

Verwunderlich war es also nicht, dass von denen, die die Patientenverfügung als verbindlich ansahen, vor allem bei unklarer Bereitschaft zur Organspende (Szenario 1b und 2b) mehr für das Beenden der intensivmedizinischen Maßnahmen votierten und eine mögliche Organspende gar nicht in Betracht zogen. Außerdem wäre zu erwarten gewesen, dass das Vorgehen neben dem Beenden der intensivmedizinischen Maßnahmen eher in Richtung eines Angehörigengespräches tendieren würde. Vor allem in Szenario 2b (baldig eintretender Hirntod), in dem die Bereitschaft zur Organspende unklar war, aber in einer Patientenverfügung lebenserhaltende Maßnahmen im Falle einer schweren irreversiblen Hirnschädigung abgelehnt wurden. Zwar wählten 34 \% derer, die der Patientenverfügung einen höheren Stellenwert einräumten, in dieser Konstellation das Beenden der intensivmedizinischen Maßnahmen, jedoch sprach sich ein ebenso großer Anteil für die begrenzte Fortführung 
der Maßnahmen aus, um den Eintritt des Hirntodes abzuwarten und anschließend ein Gespräch mit den Angehörigen zu führen (34\%). Ebenfalls bei gleichzeitig vorliegender Patientenverfügung sowie einem Organspendeausweis (Szenario 2a), entschieden sich $44 \%$ für die begrenzte Maßnahmenfortführung, obwohl sie die Patientenverfügung als verbindlich einschätzten. Bezüglich des Beendens der intensivmedizinischen Maßnahmen wurde deutlich, dass vor allem bei unklarer Bereitschaft zur Organspende und in Szenario 2b (baldig eintretender Hirntod) diejenigen das Vorgehen wählten, die die Verbindlichkeit der Verfügung anerkannten.

Welches Antwortverhalten wäre zu erwarten gewesen, wenn die Patientenverfügung als unverbindlich eingeschätzt wurde? Im Zwiespalt zwischen beiden Dokumenten (Szenario 1a und 2a) dürfte dann eher das Gespräch mit den Angehörigen oder dem Organspendeausweis entsprechend das Fortführen der intensivmedizinischen Maßnahmen erwartet werden. Bei unklarer Bereitschaft zur Organspende (Szenario 1b und 2b) wäre das Feststellen des Patientenwillens durch ein Angehörigengespräch ebenfalls sinnvoll. Dementsprechend entschieden sich diejenigen auch, die eine Patientenverfügung als unverbindlich einordneten: Sie favorisierten in Szenario 2 unabhängig von der vorliegenden Bereitschaft zur Organspende sowie auch in Szenario 1b (vermuteter Hirntod; unbekannte Organspendebereitschaft) das Angehörigengespräch (Szenario 2 je $48 \%$; Szenario 1b: $44 \%$ ). Zweitrangig wählten sie die begrenzte intensivmedizinische Maßnahmenfortführung (Szenario 2a: 38 \%; Szenario 2b: 25 \%; Szenario 1b: 30 \%). Beim Vorliegen beider Dokumente und vermutetem Hirntod (Szenario 1a) entschieden sich diejenigen, die eine Patientenverfügung als unverbindlich ansahen, etwa zu gleichen Teilen für das Gespräch und die Fortführung der Maßnahmen (46\%).

6.1.3.1.1 Hat die Patientenverfügung also Einfluss auf das gewählte Vorgehen in den Szenarien?

Die Ergebnisse zeigen, dass die Einstellung zur Patientenverfügung für das gewählte Vorgehen von Bedeutung ist (siehe Kapitel 6.1.2.2.1).

Allerdings gab es auch Widersprüchlichkeiten im Vorgehen: Diejenigen, die eine Patientenverfügung als verbindlich betrachteten, sprachen sich in Szenario 2 (baldig eintretender Hirntod) einerseits deutlich für die Realisierung einer Organspende aus, indem viele das Fortführen der intensivmedizinischen Maßnahmen wählten, andererseits stimmten auch einige gegen eine Organspende, besonders bei unklarer Bereitschaft zur Organspende (Szenario 2b). Dem Patientenwunsch nach Unterlassung lebenserhaltender Maßnahmen bei irreversibler Hirnschädigung wurde also bei dem Votum gegen die Organspende entspro- 
chen, jedoch ohne Berücksichtigung des Organspendeausweises. Diesem wurde gerecht, indem eine Fortführung der intensivmedizinischen Maßnahmen gewählt wurde, was wiederum der Patientenverfügung widersprach. Dieses in sich widersprüchliche Verhalten spiegelt die Unsicherheit und Überforderung der Befragten im Umgang mit den Dokumenten sowie auch generell im Umgang mit solchen Situationen wider. Deshalb sind einerseits die Einstellungen zu den Dokumenten, andererseits jedoch auch eine nicht zu unterschätzende Unsicherheit von Bedeutung, bezogen auf das Handeln in den unterschiedlichen Szenarien.

\subsubsection{Welchen Stellenwert hat der Organspendeausweis?}

Auch wenn nicht explizit nach dem Stellenwert des Organspendeausweises gefragt wurde, so kann auf diesen aus den Aussagen zum von der BÄK festgelegten Verfahren zur Hirntoddiagnostik indirekt geschlossen werden.

Deutlich wurde, dass von denen, die das Verfahren der BÄK als ungeeignet empfanden, die deutliche Mehrheit der Befragten das Gespräch mit den Angehörigen wählte (Szenario 1a: 50 \%; Szenario 1b: 58 \%; Szenario 2a: $42 \%$; Szenario 2b: $23 \%$ ), außer bei Szenario 2b (baldig eintretender Hirntod; unbekannte Organspendebereitschaft), wo von den Kritikern des Verfahrens das Beenden der intensivmedizinischen Maßnahmen favorisiert wurde (Szenario 2b: $42 \%$ ). Die gewählten Vorgehen sind nachvollziehbar, wenn man die kritische Einstellung zur Hirntoddiagnostik berücksichtigt. Warum sollten dann die intensivmedizinischen Maßnahmen fortgeführt werden? Es ergibt doch viel mehr Sinn, sich dann mit den Angehörigen abzustimmen oder sich gar gegen eine Organspende zu entscheiden. Dann wäre wiederum bei den Befürwortern der Diagnostik zu erwarten, dass die Mehrheit sich für das Fortführen der intensivmedizinischen Maßnahmen aussprechen würde. Zumindest, wenn die Bereitschaft zur Organspende klar war. In den Studienergebnissen war das nur teilweise zu erkennen: Lediglich beim vermuteten Hirntod und dokumentierter Bereitschaft zur Organspende (Szenario 1a) favorisierten die Zustimmenden zur Diagnostik die Maßnahmenfortführung (51\%). Sonst sprach sich die Mehrheit für das Gespräch mit den Angehörigen aus (Szenario 1b: $43 \%$; Szenario 2b: $43 \%$; Szenario 2b: $40 \%$ ). Aber es entschieden sich weniger der Befürworter gegen eine Organspende, vor allem in Szenario 2b (baldig eintretender Hirntod; unbekannte Organspendebereitschaft) und Szenario 1a (vermuteter Hirntod; dokumentierte Organspendebereitschaft). Daraus wird deutlich, dass die Befragten möglichen Unsicherheiten der Befragten im Umgang mit den Dokumenten dadurch ausweichen, dass sie das Gespräch mit den Angehörigen wählten und so die Entscheidung an die Angehörigen abgeben. 
Daneben fiel auf, dass eher zweit- oder drittrangig eine Entscheidung zugunsten des Organspendeausweises getroffen wurde, auch von den Befragten, die das Verfahren der BÄK als geeignet empfinden. Die Patientenverfügung scheint insgesamt für die Befragten einen höheren Stellenwert zu besitzen als der Organspendeausweis.

\subsubsection{Zusammenfassung des Antwortverhaltens im Zusammenhang mit den Einstellungen der Befragten}

Die Ergebnisse der Studie zeigen, dass sich vor allem die pflegenden Mitarbeiter gegen eine Organspende entscheiden, unabhängig von den unterschiedlichen Szenarien oder den vorliegenden Dokumenten. Als Gründe für die unterschiedliche Bewertung der Organspende zwischen den beiden Professionen ist die generelle Einstellung der Befragten, nicht nur hinsichtlich der beiden Dokumente, sondern auch bezogen auf die Themen Organspende und Hirntoddiagnostik zu nennen. Ferner offenbarte die Befragung ein hohes Maß an Unsicherheit bei den Teilnehmern. Diese zeigte sich u.a. darin, dass teilweise widersprüchliche und in sich unschlüssige Vorgehensweisen gewählt wurden, die mit der vorher dargelegten grundsätzlichen Einstellung der Befragten nicht übereinstimmten.

Bezüglich der Einstellung der Befragten gegenüber der Patientenverfügung war interessant, dass die Verfügung für die pflegenden Mitarbeiter bei einem Konflikt zwischen Organspendeausweis und Patientenverfügung eine größere Bedeutung hatte. Diese Gewichtung ist dadurch zu erklären, dass die Pflegenden die Patientenverfügung als verbindlich ansehen. Allerdings stellten sie eine geringere Berücksichtigung seitens der Ärztinnen und Ärzte im klinischen Alltag fest. Dadurch wird den ärztlichen Mitarbeitern letztlich möglicherweise eine mangelnde Transparenz ihrer Entscheidungen vorgeworfen. Diese hingegen empfanden die Patientenverfügung eher als unverbindlich. Das ist darauf zurückzuführen, dass Patientenverfügungen oft ungenau und nicht situationsbezogen verfasst sind, sodass dem Willen des Patienten schwer zu entsprechen ist und die Verfügungen deshalb in konkreten Einzelfällen oft unbrauchbar sind. Aus diesem Grund wichen die ärztlichen Mitarbeiter vielfach auf andere Formen der Willensbekundungen aus: den Organspendeausweis oder das Gespräch mit den Angehörigen für eine mutmaßliche Willensbekundung des Patienten.

Sowohl die ärztlichen als auch die pflegenden Mitarbeiter empfanden das Verfahren der BÄK zur Hirntoddiagnostik als geeignet, jedoch oftmals als nicht ausreichend, um den Tod des Menschen festzustellen. Für viele der Befragten spielen neben dem irreversiblen Verlust der Hirnfunktionen der Herzstillstand des hirntoten Patienten die entscheidende Rolle. Dabei lässt sich bei den Pflegenden wohl aufgrund ihrer größeren Erfahrung mit der Diag- 
nostik sowie bei der Betreuung von Organspendern eine kritischere Herangehensweise zur Hirntoddiagnostik feststellen.

$\mathrm{Zu}$ Widersprüchen kam es, wenn man die Befürwortung oder Ablehnung der These der Verbindlichkeit der Patientenverfügung sowie der These nach der Eignung des Verfahrens für die Hirntoddiagnostik in Korrelation mit dem Handeln in den Szenarien setzte. Auffällig war dabei, dass die Befürworter der Verfügungsverbindlichkeit einerseits die Realisierung einer Organspende durch Fortführung der intensivmedizinischen Maßnahmen erstrebten und sich andererseits gegen eine Organspende aussprachen. Dieser Widerspruch ist wohl mit einer Unsicherheit der Befragten im Umgang mit den beiden Dokumenten zu erklären. Bei Betrachtung der These zur Hirntoddiagnostik wurde deutlich, dass viele Befragte sich offenbar scheuen, eine eigene Entscheidung zu treffen und eher eine Absicherung bzw. jemand anderes, in diesem Falle die Angehörigen, die Entscheidung treffen lassen wollen. So sprachen sich von den Befürwortern des Verfahrens der BÄK zur Hirntoddiagnostik die Mehrheit für das Gespräch mit den Angehörigen aus.

Trotz der Unsicherheit im Umgang mit den Dokumenten und der teilweisen divergierenden Einstellung ist bei beiden Professionen aber grundsätzlich eine positive Einstellung gegenüber Organspenden festzustellen.

\subsection{Unterstützung in schwierigen Entscheidungssituationen}

Nahezu alle Befragten wünschen sich für den Fall, dass es im Zusammenhang mit dem Thema Therapiebegrenzung und Organspende zu widerstreitenden Aussagen des Patienten oder konfligierende Einschätzung verschiedener Beteiligten kommt, eine Unterstützung. Daher ist es wichtig herauszuarbeiten, wie die pflegenden und ärztlichen Mitarbeiter sich diese Unterstützung vorstellen. Was sind ihre Forderungen an eine solche Unterstützung? Reichen die schon bestehenden Hilfestellungen aus? Werden diese angenommen? Würden die Mitarbeiter entsprechend der Empfehlungen aus den Handlungsorientierungen handeln?

Diese Fragen sollen im nächsten Abschnitt beantwortet werden. Dadurch soll offengelegt werden, was für die Mitarbeitenden von Bedeutung wäre, um im Konfliktfall Handlungsmaßstäbe zu haben. 


\subsubsection{Bewertung der Verfahrensempfehlung der UMG}

Über die Hälfte der Befragten gab an, sich ethische Handlungsempfehlungen für den Umgang mit solchen Konfliktsituationen zu wünschen (60\%). Dabei liegt eine solche Verfahrensempfehlung der UMG bereits vor. Diese war jedoch zwei Dritteln der Befragten nicht bekannt. Weitere $27 \%$ hatten von der Verfahrensempfehlung gehört, kannten aber deren Inhalt nicht. Nur $5 \%$ der Befragten kannten deren Inhalt. Diese ernüchternden Zahlen sind klare Hinweise auf eine mangelnde Implementierung der Verfahrensempfehlung der UMG. Insoweit besteht dringender Handlungsbedarf, denn von denjenigen Ärzten und Pflegenden, die den Inhalt der UMG-Verfahrensempfehlung kannten, schätzten diese als „hilfreich“ oder „sehr hilfreich“ ein. Dies belegt eindrucksvoll den Nutzen der Verfahrensempfehlung der UMG.

Es muss daher das Ziel sein, die Verfahrensempfehlung der UMG bekannter zu machen, um auf diese Weise dem ärztlichen und pflegenden Personal die Entscheidungen in der Praxis zu erleichtern. Ein wichtiges Ergebnis dieser Studie ist daher, dass Maßnahmen zu deren Implementierung in den klinischen Alltag getroffen werden müssen.

\subsubsection{Diskrepanz zwischen den Empfehlungen der BÄK und der UMG und der klinischen Realität}

Inwiefern entsprechen die Empfehlungen der BÄK und der UMG aber überhaupt den Einstellungen, Handlungsweisen und Haltungen der Befragten? Diese Frage soll im Folgenden anhand der Verfahrensempfehlung der UMG diskutiert werden, da diese inhaltlich auf der Empfehlung der BÄK beruht, aber den Anspruch hat, einen stärker praxisorientierten Ansatz zu verfolgen.

\subsubsection{Diskrepanz bezogen auf Szenario 1}

Im Falle des vermuteten Hirntodes empfiehlt die Verfahrensempfehlung der UMG bei dokumentierter Organspendebereitschaft (Szenario 1a), die intensivmedizinischen Maßnahmen bis zum Abschluss der Hirntoddiagnostik und der sich ggf. daran anschließenden Organspende dem Wunsch des Patienten entsprechend fortzuführen und bei nicht dokumentierter Bereitschaft (Szenario 1b) das weiteren Vorgehen im Gespräch mit den Angehörigen zu klären. Für dieses empfohlene Vorgehen sprachen sich in der Studie unabhängig von den vorliegenden Dokumenten jeweils die Hälfte der Befragten aus (Szenario 1a: 50 \%; Szenario 1b: 53 \%). Differenzen gab es, wenn man das Antwortverhalten der beiden Professionen betrachtete: Bezogen auf Szenario 1a wählten zwei Drittel der ärztlichen (66\%) und nur $45 \%$ der pflegenden Mitarbeiter das Vorgehen aus der Empfehlung aus, bzw. 
bezogen auf Szenario 1b jeweils die Hälfte (Ärztinnen und Ärzte: 52 \%; Pflegende: 53 \%) der Pflegenden. Den Empfehlungen wurde also in Bezug auf Szenario 1a (vermuteter Hirntod; dokumentierte Organspendebereitschaft) etwas stärker von den ärztlichen Mitarbeitern entsprochen. Das lässt sich dadurch erklären, dass das Vorgehen der Fortführung der intensivmedizinischen Maßnahmen, das der Empfehlung der UMG in Szenario 1a entspricht, eine Organspende impliziert, was wie bereits in Kapitel 5.4.1 erläutert von den Pflegenden eher zurückhaltender gewählt wurde.

Insgesamt fällt also auf, dass je nach Szenario knapp die Hälfte der Befragten (Szenario 1a:50 \%; Szenario 1b: $47 \%$ mit den Empfehlungen der UMG nicht konform geht. Die Ursache dafür könnte schlicht darin liegen, dass der Inhalt der Verfahrensempfehlung der UMG nicht bekannt ist.

\subsubsection{Diskrepanz bezogen auf Szenario 2}

Im Falle des baldig eintretenden Hirntodes (Szenario 2) empfiehlt die Verfahrensempfehlung der UMG, mit dem Patientenvertreter (Betreuer oder Bevollmächtigter) zusammen zu entscheiden, ob eine Organspende dem Willen des Patienten entspräche, und zwar unabhängig davon, ob der Wille zur Organspende dokumentiert ist oder nicht. Nach der Verfahrensempfehlung soll gegebenenfalls eine Frist für die Fortsetzung der intensivmedizinischen Maßnahmen festgelegt werden. Die Antworten der in der vorliegenden Studie Befragten stehen allerdings nicht im Einklang mit dieser Verfahrensempfehlung der UMG.

Bei vorliegender Bereitschaft zur Organspende (Szenario 2a) handelten nur 43 \% der Empfehlung entsprechend und wählten das Gespräch mit den Angehörigen. Die deutliche Mehrheit der Ärztinnen und Ärzte favorisierte stattdessen eine Maßnahmenfortführung (57\%), handelte also entgegen der Empfehlung aber für eine Organspende.

Wenn die Bereitschaft des Patienten zur Organspende unbekannt war (Szenario 2b) entschieden sich ebenfalls $40 \%$ der Befragten der Empfehlung konform für das Gespräch mit den Angehörigen, jedoch deutlich mehr ärztliche (53\%) als pflegende (35\%) Mitarbeiter. Die Pflegenden sprachen sich verglichen zu den ärztlichen Kollegen eher gegen eine Organspende aus (Ärztinnen und Ärzte: 17 \%; Pflegende: 31 \%). Insgesamt ist beachtenswert, dass jeder Dritte die intensivmedizinischen Maßnahmen für einen begrenzten Zeitraum fortführen würde, um den Hirntodeintritt abzuwarten (30\%). Erst dann sollte der Frage einer möglichen Organspende mit den Angehörigen nachgegangen werden. 
6.2.2.3 Ist die Empfehlung der UMG eher Ärztinnen und Ärzten als Pflegenden bekannt? Insgesamt war auffällig, dass sich mehr der ärztlichen Mitarbeiter für das in der Verfahrensempfehlung der UMG dargelegte Vorgehen entschieden. Lediglich in Szenario 2a wählten gleich viele ärztliche wie pflegende Mitarbeiter das Angehörigengespräch (43\%), sonst sprachen sich immer über die Hälfte der Ärztinnen und Ärzte für das entsprechende Vorgehen aus (siehe Kapitel 6.2.2.2). Kannten die ärztlichen Mitarbeiter eher die Empfehlung oder handelten sie intuitiv?

Zwar hatten mehr ärztliche Mitarbeiter (35\%) von den Empfehlungen gehört, da sie sich aber weder an Inhalt erinnerten bzw. die Empfehlung nicht gelesen hatten, spielte das kaum eine Rolle (Pflegende: 27 \%). Erstaunlicherweise waren unter den wenigen, die die Empfehlung und vor allem den Inhalt kannten (5\%), mehr Pflegende (6\%) als Ärztinnen und Ärzte (3\%). Demzufolge war hinsichtlich der Entscheidung zum Vorgehen in den verschiedenen Szenarien weniger die Kenntnis der Verfahrensempfehlung als die allgemeine Einstellung von Bedeutung.

6.2.2.4 Gibt es keinen Bedarf für diese Empfehlungen, da die Mitarbeiter sowieso intuitiv empfehlungsgetreu handeln?

Ein Handeln, das nicht mit den Empfehlungen der UMG übereinstimmt, darf nicht als falsch angesehen werden. Bei der Verfahrensempfehlung handelt es sich lediglich um eine Handlungsorientierung, die als Hilfestellung, nicht aber als bindend angesehen werden soll. Vor allem unter dem Gesichtspunkt, dass nur wenige ärztliche und pflegende Mitarbeiter die Empfehlung kannten, ist es interessant, dass trotzdem vor allem die Ärztinnen und Ärzte so handeln, wie es in dem Dokument empfohlen wird. Ist also eine schriftliche Festlegung von Kriterien zur Handlungsorientierung unnötig?

Ganz im Gegenteil: Neben dem Wunsch nach einer solchen Hilfestellung seitens der Befragten selbst machen auch die Studienergebnisse deutlich, dass eine ethische Handlungsanleitung von großem Nutzen wäre. Auch wenn die Ärztinnen und Ärzte größtenteils der Empfehlung entsprechend handeln, scheint es trotzdem noch viel Unklarheit bezüglich der Konfliktlösung zu geben. Gleichwohl ist hervorzuheben, dass die ärztlichen Mitarbeiter einer Organspende eher nicht im Weg stehen, sondern versuchen, diese einzuleiten und durchzuführen. Anders verhält es sich mit einem Teil der Pflegenden. Von einem Teil der Pflegenden wird der Patientenwille zur Organspende nicht als ausschlaggebend für ihr Handeln betrachtet und durch das Unterlassen der intensivmedizinischen Maßnahmen eine Organspende unmöglich gemacht. Aber auch bei unbekannter Bereitschaft zur Organ- 
spende suchten allerdings erstaunlich viele pflegende Mitarbeiter gar nicht erst den Dialog mit den Angehörigen, sondern favorisierten sofort die Beendigung der Maßnahmen.

Aufgrund dieser Ergebnisse scheint es sehr sinnvoll zu sein, den ärztlichen sowie auch den pflegenden Mitarbeitern Aufklärung und Unterstützung bei dieser Thematik zu bieten. Angesichts des bestehenden erheblichen Organmangels in Deutschland (Kapitel 2.3.4) sollte das medizinische Personal eine Organspende nicht verhindern, sondern vielmehr die Möglichkeit einer Spende in Betracht ziehen, diese kommunizieren und realisierende Schritte einleiten.

\subsubsection{Weitere erwünschte Unterstützung}

Neben schriftlichen Handlungsempfehlungen wünschten sich die Studienteilnehmer vor allem Ansprechpartner, die sowohl generell in konflikthaften Situationen als auch insbesondere im Gespräch mit den Angehörigen beratend und moderierend hinzugezogen werden können. Darüber hinaus forderten vor allem die Ärztinnen und Ärzte rechtliche Hintergrundinformationen.

\subsubsection{Bevorzugte Ansprechpartner der Pflegenden}

Die Art der gewünschten Ansprechpartner divergierte zwischen den beiden Professionen. Deutlich mehr Pflegende als Ärztinnen und Ärzte wünschten sich Ansprechpartner für ein Gespräch mit den Angehörigen. Die ärztlichen Mitarbeiter konnten sich eher generell Berater oder Moderator für konflikthafte Situationen an sich vorstellen.

Woran könnte das liegen? Vor allem auf Intensivstationen haben Pflegende oft engen Kontakt zu den Angehörigen. Pflegende sind neben der Betreuung des Patienten auch für die Unterstützung der Angehörigen verantwortlich (Kuhlmann 2004). Mehr noch, die Intensivpflegekraft wird als die Person angesehen, die am ehesten in der Lage ist, die Bedürfnisse und Wünsche der Angehörigen zu erfüllen (Hickey und Lewandowski 1988). Die Pflegenden verfügen dabei aber im Gegensatz zu den Ärztinnen und Ärzten über keine Entscheidungsgewalt und stehen deshalb bei der Ausübung ihrer Rolle immer wieder vor Schwierigkeiten. Sie befürchteten beispielsweise, dass die ärztlichen Kollegen anschließend Aussagen machen, die mit ihren eigenen in Widerspruch stehen. Neben diesem Mangel an Autorität im Vergleich zu den ärztlichen Mitarbeitern erschweren auch Unsicherheiten im Umgang mit emotionalen und psychosozialen Bedürfnissen der Angehörigen den Umgang mit ihnen (Hickey und Lewandowski 1988). Dementsprechend ist es nicht verwunderlich, dass die Pflegenden als Bezugspersonen der Angehörigen mehr Unterstützung im Gespräch mit den Angehörigen wünschen. 


\subsection{Bevorzugte Ansprechpartner der ärztlichen Mitarbeiter?}

Ärztinnen und Ärzte hingegen wünschen sich eher Ansprechpartner, die generell in konflikthaften Situationen beratend oder moderierend fungieren würden. Warum unterscheiden sich die Forderungen hinsichtlich der Professionen? Dazu muss zuerst die Rolle des ärztlichen Personals bei einer Organspende begutachtet werden. Laut Transplantationsgesetz ist der Transplantationsbeauftragte u. a. zuständig die angemessene Begleitung der Angehörigen ( $\int 9 b$ Abs. 2 Satz 2 TPG). Kümmern sich in der Praxis also die Transplantationsbeauftragten um die Kommunikation mit den Angehörigen? Nein, in der Regel sind es Ärztinnen und Ärzte, selten in Begleitung von Pflegenden. Die Kommunikation bzw. ein Leitfaden für das Gespräch ist im Gesetz nicht verankert und lässt somit Spielraum für den klinischen Alltag (Ethikrat.org - Hirntod und Entscheidung zur Organspende: Stellungnahme 2015). Dabei wird, wie bereits in Kapitel 2.1 dargelegt, heutzutage das Prinzip des „informed consent" zur Behandlung moralischer Probleme genutzt. Die Ärztinnen und Ärzte sollen demnach Empfehlungen aussprechen und aufklären, um so bei der auf Verständnis beruhenden Entscheidungsformung des Patienten bzw. der Angehörigen (nach mutmaßlichem Willen der Patienten) zu helfen (Kapitel 2.1). Die Beteiligung des Patienten bzw. der Angehörigen erfordert gerade im Umgang mit dieser belastenden, konfliktpotenziellen und sehr emotionalen Thematik eine gewisse Kommunikationsfertigkeit der ärztlichen Mitarbeiter, auf die sie allerdings nicht zurückgreifen können, weil sie diese oft nicht erlernt haben (Klemperer 2003).

\subsubsection{Rechtliche Hintergrundinformationen}

Ferner wünscht sich die Hälfte der Ärztinnen und Ärzte Informationen zum rechtlichen Hintergrund in Konfliktfällen (Ärztinnen und Ärzte: $52 \%$; Pflegende: $41 \%$ ). Dies ist damit zu erklären, dass sie in erster Linie die rechtliche Verantwortung für die getroffenen Entscheidungen tragen. Trotz einer gesetzlichen Regelung gibt es keine umfassende Rechtssicherheit (Ulsenheimer et al. 2014), was zu Unsicherheiten im Umgang mit Patientenverfügungen und in der Folge zu dem Wunsch nach rechtverbindlicher Information führen kann. Zu beachten ist dabei jedoch, dass Pflegende zwar in der Regel keine Entscheidungs-, sehr wohl aber eine Übernahme- und Durchführungsverantwortung haben. Auch ist zu beachten, dass die Weisungsgebundenheit der Pflegekraft dort endet, wo für sie zu erkennen ist, dass die ärztliche Anordnung eindeutig rechtswidrig ist, z. B. weil sie dem erklärten Willen des Patienten eindeutig widerspricht. In diesem Fall hat die Pflegekraft eine sog. Remonstrationspflicht, d. h. sie muss ihre Bedenken gegen die Rechtmäßigkeit der dienstlichen Anordnung unverzüglich bei ihrem unmittelbaren Vorgesetzten geltend ma- 
chen (Schell 2005). Rechtliche Informationen zur Behandlung der dargelegten Konfliktsituationen sind also auch für Pflegende nicht irrelevant.

\subsubsection{Zusammenfassung zur Unterstützung in schwierigen Entscheidungs- situationen}

Einig waren sich alle Befragten darüber, dass eine Form der Unterstützung für Konfliktsituationen zwischen dem gleichzeitigen Vorliegen eines Organspendeausweiseses und einer Patientenverfügung von großem Nutzen wäre. Hinsichtlich der Unterstützung gab es zwei Arten, die bei den Befragten besonders vorstellbar waren: ethische Handlungsempfehlungen oder Ansprechpartner für Gespräche.

Ethische Handlungsempfehlungen liegen in Form der Verfahrensempfehlung der UMG oder dem Arbeitspapier der BÄK vor, waren aber weitgehend unbekannt. Bei den Befragten, die die Verfahrensempfehlung der UMG kannten, stieß diese auf ausschließlich positive Resonanz. Die Empfehlung kann also eine Hilfestellung sein und sollte deshalb bekannter gemacht werden. Trotz der Unbekanntheit oder gerade deshalb war es von Interesse herauszufinden, ob das gewählte Handeln der Befragten mit dem in der Verfahrensempfehlung vorgeschlagenen übereinstimmt. Dabei ergab sich, dass die Teilnehmenden größtenteils nicht entsprechend dem Vorgehen in der Empfehlung der UMG gehandelt hätten: Vor allem bei einem baldig eintretendem Hirntod (Szenario 2) entschieden mit knapp $40 \%$ (Szenario 2a: 43 \%; Szenario 2b: $40 \%$ ) etwas weniger Teilnehmende der Empfehlung entsprechend, als bei Szenario 1 (vermuteter Hirntod), in dem sich knapp die Hälfte der Befragten so entschied (Szenario 1a: 50 \%; Szenario 1b: 53 \%). Im Hinblick auf die Professionen fiel auf, dass sich eher die ärztlichen Mitarbeiter der Verfahrensempfehlung konform aussprachen. Dies kann auf die allgemeine Einstellung der ärztlichen Mitarbeiter und auch auf ihre allgemeine Erfahrenheit zur Thematik zurückgeführt werden, wobei die Erfahrung sich nur teilweise auf den Umgang mit Organspende, Hirntoddiagnostik und Patientenverfügung bezieht, sondern eher Bezug nimmt auf die generelle Rolle der ärztlichen Mitarbeiter, die im klinischen Alltag als Entscheidungsträger handeln müssen.

Aufgrund dieser Verantwortung der Ärztinnen und Ärzte war es auch nicht verwunderlich, dass sie sich rechtliche Hintergrundinformationen wünschten. Auch der Nutzen von Ansprechpartnern zur Unterstützung in Gesprächen, den sich beide Professionen vorstellen konnten, war für die Ärztinnen und Ärzte eher ein Berater oder Moderator für konfliktreiche Situationen an sich von Interesse. Zur Entscheidungsfindung in dieser sehr emotionalen Phase scheint ein solcher Ansprechpartner eine Hilfe für die ärztlichen Mitarbeiter darzustellen. Die Pflegenden hingegen, die zwar eine engere Bindung zu den Angehörigen 
haben, aber nicht in den Prozess der Entscheidung und somit auch eher weniger in konfliktreiche Situationen eingebunden sind, wünschen sich eher Personen, die zu Gesprächen mit Angehörigen hinzugezogen werden können.

\subsection{Beantwortung der Forschungsfragen}

In diesem Abschnitt wird nochmal besonders auf die in Kapitel 3 formulierten Forschungsfragen eingegangen und jede einzeln beantwortet. Da die Ergebnisse im vorangegangenen Teil der Arbeit bereits ausführlich dargestellt wurden (Kapitel 5), werden die Fragen hier nur knapp und zusammenfassend beantwortet.

\subsubsection{Wie ist die allgemeine Einstellung der ärztlichen und pflegenden Mitarbeitenden der Intensivstationen zu den Themen Organtransplantation, Hirntoddiagnostik und Patientenverfügung?}

Sowohl der Patientenverfügung als auch der Organspende wurde von den Teilnehmenden eine wichtige Rolle im klinischen Alltag zugerechnet. Es gab aber auch differierende Einschätzungen. So war die Festlegung des Hirntodkriteriums umstritten, obwohl die von der BÄK festgelegte Hirntoddiagnostik mehrheitlich als geeignet empfunden wurde. Nur die Hälfte der Befragten teilten die Einschätzung der BÄK, dass der Hirntod als Tod des Menschen anzusehen ist (siehe Kapitel 5.3.1). Bezüglich der Patientenverfügung gab es Unterschiede in der Einschätzung durch die befragten Professionen. Die ärztlichen Mitarbeiter verstanden die Patientenverfügung eher als unverbindliche Orientierung, die sie aber trotzdem im praktischen Alltag in der Regel berücksichtigen, die Pflegenden hingegen sahen die Patientenverfügung eher als verbindlich an, gaben aber zugleich an, dass diese in der Praxis nach ihrer Beobachtung oftmals unberücksichtigt bleibt (siehe Kapitel 5.3.2).

\subsubsection{Wie sehen die Mitarbeitenden den möglichen Konflikt zwischen Patientenverfügung und Organspendeerklärung?}

Für die Befragten scheint der Konflikt zwischen den beiden Willensbekundungen der Patienten eine Rolle im klinischen Alltag zu spielen. Ersichtlich wurde das daran, dass sich nahezu alle Befragten Hilfe für solche Konfliktsituationen wünschten (Kapitel 5.6.1). Der Wunsch nach Hilfestellung im Umgang mit solchen Konflikten lässt auch auf eine Unsicherheit bzw. Überforderung der Teilnehmer schließen, die sich auch in den Szenarien wiederspiegelt: Vor allem bei Szenario 2b, bei dem der Eintritt des Hirntodes innerhalb der nächsten Tage vermutet wird und die Bereitschaft zur Organspende unklar ist, konnten sich die Befragten nicht entscheiden und wählten zu jeweils einem Drittel die Fortführung 
der intensivmedizinischen Maßnahmen, das Angehörigengespräch oder das Beenden der Maßnahmen. Drei komplett unterschiedliche Vorgehen, die einerseits eine Organspende ermöglichen und sie andererseits verhindern (Kapitel 5.4.2).

Der Konflikt zwischen der Patientenverfügung und dem Organspendeausweis scheint also vorhanden zu sein und Unterstützung dabei nötig und erwünscht.

\subsubsection{Welcher Willenserklärung (Wunsch nach Therapiebegrenzung oder Bereitschaft zur Organspende) würden die Mitarbeitenden im Falle des vermuteten Hirntods den Vorrang geben? Welcher im Falle eines erwarteten Hirntodes?}

Unabhängig vom Eintritt des Hirntods wählten die Teilnehmer bei den Szenarien Vorgehen, die eine Organspende realisieren würden. Man könnte also davon ausgehen, dass sowohl beim vermuteten, als auch beim erwarteten Hirntod der Organspendeausweis präferiert wurde. Allerdings ist das nicht ganz richtig, da eigentlich nur die Antwort der Fortführung der intensivmedizinischen Maßnahmen zur Hirntoddiagnostik dem Handeln gemäß eines Organspendeausweises entspräche. Demzufolge wurde der Ausweis immer dann einer Patientenverfügung vorgezogen, wenn die Bereitschaft zur Organspende dokumentiert war (Szenarien a) - unabhängig vom Eintritt des Hirntodes. Sobald keine Bereitschaft zur Organspende dokumentiert war (Szenarien b), spielte der Eintritt des Hirntods jedoch wieder eine maßgebliche Rolle: Beim vermuteten Hirntod (Szenario 1b) sollte das weitere Vorgehen der Mehrheit entsprechend mit den Angehörigen besprochen werden. Die Patientenverfügung wurde also berücksichtigt, aber auch die Möglichkeit einer Organspende, obwohl der Wille des Patienten dazu gar nicht dokumentiert war (siehe Kapitel 5.4.1). Beim erwarteten Hirntod (Szenario 2b) wählten die Teilnehmer zwar auch das Angehörigengespräch, aber zu nahezu gleichen Teilen zwei andere Vorgehen: Erstens die begrenzte Fortführung der intensivmedizinischen Maßnahmen, um den Eintritt des Hirntodes abzuwarten und dann ein Gespräch mit den Angehörigen zu führen. Die Entscheidungsgewalt würde insofern wiederum bei den Angehörigen liegen, aber durch die Maßnahmenfortführung wird eher die Möglichkeit einer Organspende der Patientenverfügung vorgezogen. Zweitens votierten die Befragten bei Szenario 2b (baldig eintretender Hirntod; unklare Organspendebereitschaft) für das Beenden der intensivmedizinischen Maßnahmen und entsprachen somit der Patientenverfügung, in der lebenserhaltende Maßnahmen abgelehnt wurden. Wurde also nur beim erwarteten Hirntod die Patientenverfügung der Organspende vorgezogen? Zwar nicht mehrheitlich, aber es sprachen sich dennoch einige (vor allem pflegende Mitarbeiter) für eine Beendigung der intensivmedizinischen Maßnahmen 
und somit für die Patientenverfügung aus. Wie bereits erwähnt, vor allem beim erwarteten Hirntod (Szenario 2), aber auch unabhängig vom Eintritt des Hirntods, sobald nämlich die Bereitschaft des Patienten zur Organspende unklar war (Szenarien b)(siehe Kapitel 5.4.2).

Sowohl beim vermuteten als auch beim erwarteten Hirntod wurde also der Organspendeausweis einer Patientenverfügung gegenüber präferiert, sobald ein Organspendeausweis vorlag. Wenn die Bereitschaft zur Organspende unklar war, wurden beide Willensbekundungen berücksichtigt, indem die Entscheidung über eine Organspende an die Angehörigen abgetreten wurde. Neben dem Angehörigengespräch wurde beim erwarteten Hirntod (Szenario 2b) jeweils noch die Patientenverfügung (Beenden der intensivmedizinischen Maßnahmen) oder der Organspendeausweis (Fortführung der intensivmedizinischen Maßnahmen) benutzt.

\subsubsection{Macht es für die Mitarbeitenden bei der Bewertung des möglichen Konflikts einen Unterschied, ob der Wille des Patienten in einer Patientenverfügung bzw. Organspendeerklärung schriftlich dokumentiert oder stellvertretend von den Angehörigen vorgetragen wird?}

Für die Teilnehmer der Studie schien es einen Unterschied zu machen, ob der Wille des Patienten in schriftlicher Form vorlag oder mittels der Angehörigen vorgetragen wurde: Gerade in Situationen, in denen nur eine Willensbekundung des Patienten vorlag, wie beispielsweise die Patientenverfügung in Szenario $1 \mathrm{~b}$ und 2b, wichen die Befragten oft auf ein Gespräch mit den Angehörigen aus, in dem der Wille des Patienten nochmal evaluiert werden sollte. Auch im Konflikt zwischen der Patientenverfügung und dem Organspendeausweis räumten einige Teilnehmer der Meinung der Angehörigen einen hohen Stellenwert ein und wählten somit das Gespräch mit den Angehörigen (siehe Kapitel $5.4)$.

Demzufolge wurde deutlich, dass nicht nur im Konflikt zwischen der Patientenverfügung und dem Organspendeausweis, sondern auch beim Vorliegen nur einer dieser Willensbekundungen des Patienten, viele Mitarbeiter der UMG eine Rückversicherung durch die Angehörigen erstreben.

\subsubsection{Hat die Einstellung der Befragten zu den Themen Organspende und Patientenverfügung einen Einfluss auf das Handeln bei den Szenarien?}

Unabhängig von der persönlichen Einstellung zur Organspende und zur Patientenverfügung, sprach sich eine Mehrheit der Befragten für eine Organspende aus. Bei genauerer Betrachtung fällt allerdings auf, dass die Kritiker der Organspende und der Patientenverfü- 
gung sich eher gegen eine Organspende aussprachen, indem sie tendenziell häufiger für das Beenden der intensivmedizinischen Maßnahmen wählten. Insofern hatte die persönliche Einstellung der Befragten Einfluss auf die Bewertung der Szenarien (siehe Kapitel 5.4.4.6).

\subsubsection{Gibt es Differenzen zwischen den ärztlichen und pflegenden Mitarbeitern hinsichtlich der Einstellung zu den Themen Organspende und Patientenverfügung oder zu dem Vorgehen bei den Szenarien?}

Die Organspende und die Patientenverfügung wurden sowohl von den ärztlichen als auch von den pflegenden Mitarbeitern als im klinischen Alltag relevant eingeschätzt. Allerdings zeigten sich bei beiden Themen Unterschiede hinsichtlich der Einstellung: Die Pflegenden sahen die mit der Organspende einhergehenden Handlungen (Hirntoddiagnostik, Hirntodkriterium und Organtransplantation) etwas kritischer als die ärztlichen Kollegen (siehe Kapitel 5.3.1). Darüber hinaus sahen die pflegenden Mitarbeiter die Patientenverfügung häufiger als verbindliches Dokument an, waren aber zugleich der Ansicht, dass es in der Praxis oftmals nicht berücksichtigt wird.. Die Ärztinnen und Ärzte waren der Ansicht, dass die Patientenverfügung in der Praxis in der Regel berücksichtigt wird, auch wenn sie das Dokument häufiger als nicht verbindlich ansahen (siehe Kapitel 5.3.2).

Aufgrund dieser Einstellungsunterschiede lassen sich auch die Unterschiede im Antwortverhalten in den Szenarien erklären. Im Vergleich zu den Ärztinnen und Ärzten votierten die Pflegenden insgesamt zurückhaltender für Maßnahmen, die eine Organspende realisieren würden. Mehr noch, sie sprachen sich sogar häufiger gegen eine Organspende aus, da sie den Willen in der Patientenverfügung berücksichtigt sehen wollten und dem Thema Organspende kritischer gegenüber standen (siehe Kapitel 5.4.3).

\subsubsection{Sind das Arbeitspapier der BÄK und die Verfahrensempfehlung der UMG den Mitarbeitenden bekannt? Und wenn ja, als wie hilfreich werden diese eingestuft?}

Die auf dem Arbeitspapier der BÄK basierende Verfahrensempfehlung der UMG war den Teilnehmern dieser Studie weitestgehend nicht bekannt. Die wenigen, die die Empfehlung und ihren Inhalt kannten, schätzten sie ausnahmslos als hilfreich ein (siehe Kapitel 5.6.2).

Leider musste aufgrund eines Formatierungsfehlers des Fragebogens die entsprechende Frage zum Arbeitspapier der BÄK verworfen werden. 


\subsubsection{Wünschen sich die Mitarbeitenden Unterstützung bei}

Entscheidungskonflikten im Zusammenhang mit Patientenverfügung und Organspendeerklärung? Wenn ja, in welcher Form?

Deutlich wurde, dass nahezu alle der befragten ärztlichen und pflegenden Mitarbeiter sich Unterstützung für eine klinische Entscheidungsfindung im Rahmen solcher Konflikte wünschten. Favorisiert wurden dabei kompetente Ansprechpartner als Unterstützung für Gespräche mit Angehörigen oder für konflikthafte Situationen. Ebenfalls wurde der Wunsch nach ethischen Handlungsempfehlungen und mehr rechtlichen Hintergrundinformationen geäußert (Kapitel 5.6.1).

\subsubsection{Welchen Einfluss haben Faktoren wie Alter, Geschlecht, Religionszugehörigkeit, berufliche Funktion oder Fachbereich (Klinikzugehörigkeit) auf das Antwortverhalten?}

Die genannten soziodemografischen Faktoren hatten keinen signifikanten Einfluss auf das Antwortverhalten. 


\section{$7 \quad$ Zusammenfassung und Ausblick}

Die Themen der Therapiebegrenzung und Organspende sind Bestandteil des klinischen Alltags von ärztlichen und pflegenden Mitarbeitern auf Intensivstationen. Immer mehr Patienten verfügen über eine Patientenverfügung oder einen Organspendeausweis. Zu Problemen kommt es, wenn Patienten über beide Dokumente verfügen und diese widersprechende Aussagen enthalten. Der daraus resultierende Konflikt stellt sich im Klinikalltag immer wieder.

Die im Rahmen dieser Arbeit durchgeführte Studie machte deutlich, dass bei den behandelnden Personen große - auch rechtliche - Unsicherheiten im Umgang mit dem genannten Konflikt bestehen. Diese gehen so weit, dass ein Teil der befragten Mitarbeitenden der Göttinger Intensivstationen die Möglichkeit einer Organspende auch dann nicht in Betracht ziehen würde, wenn die Bereitschaft des Patienten zur Organspende schriftlich dokumentiert ist. Patientenverfügung und Organspendeausweis stellen Formen des erklärten Patientenwillens dar, die bei der Entscheidung über eine möglich Fortsetzung einer intensivmedizinischen Maßnahme bei vermutetem bzw. erwartetem Hirntod zu beachten sind. Die Studie konnte zeigen, dass im Falle konfligierender Aussagen viele der - insbesondere der pflegenden - Mitarbeitenden von Intensivstationen eher dem in der Patientenverfügung dokumentierten Wunsch nach Therapiebegrenzung als der im Organspendeausweis niedergelegten Bereitschaft zur Organspende folgen würden.

Die Studie machte ferner deutlich, dass es Handlungsbedarf gibt. Dieser besteht insbesondere dahingehend, dass die ärztlichen und pflegenden Mitarbeiter im Konflikt im Umgang mit den beiden Willensbekundungen des Patienten unterstützt werden müssen. Die dazu schon bestehende Form der Unterstützung, nämlich die Verfahrensempfehlung der UMG, wird von denjenigen als hilfreich angesehen, die sie kennen. Leider ist dies nur eine kleine Minderheit der Mitarbeitenden. Dies spricht dafür, die Empfehlung stärker bekannt zu machen und in den Klinikalltag zum implementieren. Darüber hinaus sollten in den Kliniken Ansprechpartner vorhanden sein, die entweder als Unterstützung in Angehörigengesprächen fungieren oder von den ärztlichen und pflegenden Mitarbeitern in konfliktreichen Situationen moderierend und beratend hinzugezogen werden können. 
Zielführend wäre es sicherlich auch, Patienten, die sowohl über eine Patientenverfügung als auch über einen Organspendeausweis verfügen, möglichst früh auf den dadurch möglichen Konflikt hinzuweisen. Hilfsweise könnte dieser Hinweis gegenüber den Angehörigen erfolgen. Sicherlich wäre es auch interessant zu erfahren, in welchem Maße dieser Konflikt für die Patienten bzw. deren Angehörigen eine Rolle spielt. Dazu wären einerseits die Patienten selber und andererseits auch die Angehörigen zu befragen, da diese oft den mutmaßlichen Willen des Patienten bestimmen müssen. Ergänzend wäre eine detaillierte Befragung zur Verfahrensempfehlung der BÄK seitens der ärztlichen und pflegenden Mitarbeiter durchzuführen. 


\section{Anhang}

\subsection{Der Fragebogen}

\section{Befragung zum Thema „Patientenverfügung und Organspendeerklärung“}

Bitte schicken Sie den ausgefüllten Fragebogen bis spätestens 03.04.2015 an folgende Adresse:

Akademie für Ethik in der Medizin

Humboldtallee 36

37073 Göttingen

HAUSPOST

\section{Allgemeine Fragen zur postmortalen Organspende, Organtransplantation und} Hirntoddiagnostik

1.1 Nach Angaben der Deutschen Stiftung Organtransplantation ist die Zahl der postmortalen Organspenden in Deutschland in den letzten Jahren rückläufig.

1.1.1 Wie beurteilen Sie diese Entwicklung?

$\square$ Als vorübergehenden Trend

$\square$ Als längerfristigen Trend

$\square$ Weiß nicht

1.1.2 Was sind Ihrer Meinung nach mögliche Gründe für den Rückgang der postmortalen Organspenden in Deutschland? (Mehrfachnennung möglich)

Die in den letzten Jahren bekannt gewordenen Skandale in der Transplantationsmedizin

Mangelnde Aufklärung der Öffentlichkeit

Mangelnde finanzielle Entschädigung der Spenderkrankenhäuser

Mangelnde Kooperation zwischen den Spenderkrankenhäusern und den Transplantationszentren 
Die gesetzliche Regelung der Organspende in Deutschland

Ethische Bedenken gegenüber dem Hirntodkriterium

Zweifel an der Sicherheit der Hirntoddiagnostik

$\square$ Die wachsende Zahl an Patientenverfügungen

Sonstige,

und

zwar:

1.2 Hatten Sie in Ihrer bisherigen beruflichen Tätigkeit bereits mit dem Thema postmortale Organspende zu tun?

$\square$ Ja, und zwar: (Mehrfachnennung möglich)

$\square$ Im Zusammenhang mit der Betreuung von Organspendern

$\square$ Im Zusammenhang mit der Betreuung von Organempfängern

$\square$ Im Zusammenhang mit der Hirntoddiagnostik

$\square$ Auf sonstige Weise, und zwar:

Nein

1.3 Fühlen Sie sich mit den Kriterien zur Durchführung einer Hirntoddiagnostik vertraut?
$\square \mathrm{Ja}$
Nein
Weiß nicht

1.4 Waren Sie selbst schon einmal bei einer Hirntoddiagnostik mit dabei (oder haben diese selbst durchgeführt)?
$\square \mathrm{Ja}$
$\square$ Nein
Weiß nicht

1.5 Verfügen Sie selbst über einen Organspendeausweiß?
$\square \mathrm{Ja}$
Nein
Weiß nicht

1.6 Verfügen Sie selbst über eine Patientenverfügung?
$\square \mathrm{Ja}$
Nein
Weiß nicht 


\section{Fragen zur allgemeinen Einstellung gegenüber Organtransplantation, Hirntod- diagnostik und Patientenverfügung}

2.1 Wie stehen Sie zu folgenden Aussagen zur Organtransplantation und Hirntoddiagnostik?

$\begin{array}{cccc}\text { Stimme } & \text { Stimme } & \text { Stimme } & \text { Stimme } \\ \text { voll und } & \text { eher } & \text { eher nicht } & \text { überhaupt } \\ \text { ganz } \mathrm{zu} & \mathrm{zu} & \mathrm{zu} & \text { nicht } \mathrm{zu}\end{array}$

Organtransplantation ist eine wichtige Möglichkeit, schwerkranken Menschen zu helfen

Das von der Bundesärztekammer festgelegte Verfahren zur Hirntoddiagnostik ist geeignet, den Hirntod als irreversiblen Ausfall aller Hirnfunktionen sicher festzustellen

Der Hirntod ist mit dem Tod des Menschen gleichzusetzen

Hirntote Patienten sind erst dann wirklich tot, wenn das Herz nach Abschalten der Geräte zu schlagen aufgehört hat

2.1 Wie stehen Sie zu folgenden Aussagen zur Patientenverfügung?

$\begin{array}{cccc}\text { Stimme } & \text { Stimme } & \text { Stimme } & \text { Stimme } \\ \text { voll und } & \text { eher } & \text { eher nicht } & \text { überhaupt } \\ \text { ganz zu } & \mathrm{zu} & \mathrm{zu} & \text { nicht zu }\end{array}$

Die Zahl der Patientenverfügungen hat in den letzten Jahren merklich zugenommen

Patientenverfügungen werden in den letzten Jahren bei klinischen Entscheidungen stärker berücksichtigt

Patientenverfügungen sind keine verbindlichen Handlungsanweisungen, sondern geben einen Hinweis darauf, was der Patient gewollt haben könnte 


\section{$\underline{3 \text { Fragen zum Verhältnis von Patientenverfügung und Organspendeerklärung }}$}

Wie würden Sie in den nachfolgenden Situationen entscheiden?

3.1 Stellen Sie sich folgende Situation vor (Szenario 1):

Ein Patient liegt nach einem schweren Verkehrsunfall mit einer Hirnblutung auf der Intensivstation. Er ist bewusstlos und wird maschinell beatmet. Die behandelnden Ärzte vermuten, dass der Hirntod bereits eingetreten ist.

Der Patient hat eine Patientenverfügung, in der er lebenserhaltende Maßnahmen für den Fall einer schweren irreversiblen Hirnschädigung ablehnt.

Szenario 1a: Der Patient hat seine Bereitschaft zur Organspende in einem Organspendeausweis dokumentiert.

3.1.1 Für welches Vorgehen würden Sie sich aussprechen?

Die intensivmedizinischen Maßnahmen sollen fortgeführt werden, um eine Hirntoddiagnostik durchführen zu können.

Das weitere Vorgehen soll mit den Angehörigen besprochen werden.

Die intensivmedizinischen Maßnahmen sollen beendet werden.

$$
\text { Sonstiges, und zwar: }
$$

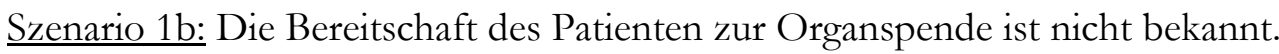

\subsubsection{Für welches Vorgehen würden Sie sich aussprechen?}

Die intensivmedizinischen Maßnahmen sollen fortgeführt werden, um eine Hirntoddiagnostik durchführen zu können. Im Anschluss daran soll die Frage einer möglichen Organspende mit den Angehörigen besprochen werden.

Das weitere Vorgehen (einschl. der Frage einer möglichen Organspende) soll mit den Angehörigen besprochen werden.

Die intensivmedizinischen Maßnahmen sollen beendet werden.

$$
\text { Sonstiges, und zwar: }
$$


3.2 Stellen Sie sich folgende Situation vor (Szenario 2):

Ein Patient liegt nach einem schweren Verkehrsunfall mit einer Hirnblutung auf der Intensivstation. Er ist bewusstlos und wird maschinell beatmet. Die behandelnden Ärzte vermuten, dass der Hirntod innerhalb der nächsten Tage eintreten wird.

Der Patient hat eine Patientenverfügung, in der er lebenserhaltende Maßnahmen für den Fall einer schweren irreversiblen Hirnschädigung ablehnt.

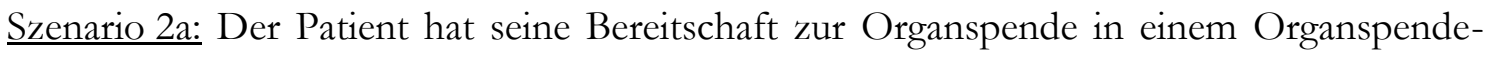
ausweis dokumentiert.

3.2.1 Für welches Vorgehen würden Sie sich aussprechen?

Die intensivmedizinischen Maßnahmen sollen für einen begrenzten Zeitraum fortgeführt werden, um den Eintritt des Hirntodes abzuwarten.

Das weitere Vorgehen soll mit den Angehörigen besprochen werden.

Die intensivmedizinischen Maßnahmen sollen beendet werden.

Sonstiges,

und

zwar:

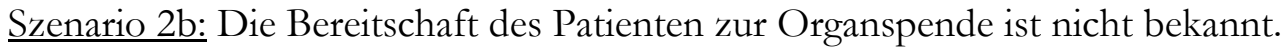

3.2.2 Für welches Vorgehen würden Sie sich aussprechen?

Die intensivmedizinischen Maßnahmen sollen für einen begrenzten Zeitraum fortgeführt werden, um den Eintritt des Hirntodes abzuwarten. Nach Feststellung des Hirntodes soll die Frage einer möglichen Organspende mit den Angehörigen besprochen werden.

Das weitere Vorgehen (einschl. der Frage einer möglichen Organspende) soll mit den Angehörigen besprochen werden.

$\square$ Die intensivmedizinischen Maßnahmen sollen beendet werden.

Sonstiges, und

zwar: 
Wenn Sie sich in den Szenarien 2a oder 2b für eine zeitlich begrenzte Fortführung der intensivmedizinischen Maßnahmen (Antwortoption 1) ausgesprochen haben:

3.3 Welcher Zeitraum wäre für Sie ethisch vertretbar:

$\square \max$. Tage

$\square$ gar keine Fortführung

Sonstiges,

und

zwar:

\section{Unterstützung bei der klinischen Entscheidungsfindung}

4.1 Stellen Sie sich vor, eines der oben genannten Szenarien würde sich auf Ihrer Station ereignen. Würden Sie sich in diesem Fall Unterstützung wünschen?

Ja, und zwar (Mehrfachnennung möglich):

Allgemeine Informationen zum rechtlichen Hintergrund

Ethische Handlungsempfehlungen für die einzelnen Szenarien

Kompetente Ansprechpartner, die zu den Angehörigengesprächen hinzugezogen werden können.

$\square$ Kompetente Ansprechpartner, die in konflikthaften Situationen beratend bzw. moderierend hinzugezogen werden können.

$\square$ Sonstiges, und zwar:

$\square$ Nein

4.2 Die Bundesärztekammer hat 2013 Empfehlungen zum Umgang mit Patientenverfügung und Organspendeerklärung veröffentlicht.

4.2.1 Sind Ihnen diese Empfehlungen bekannt?

$\square$ Nein, ich habe von diesen Empfehlungen noch nie gehört.

Ja, ich habe von diesen Empfehlungen gehört. Ich habe sie aber nicht gelesen bzw. kann mich nicht mehr an deren Inhalt erinnern

$\square$ Ja, der Inhalt dieser Empfehlungen ist mir bekannt. 
4.2.2 Empfinden Sie diese Empfehlungen als hilfreich? (Bitte kreuzen Sie die zutreffende Antwort an)

Sehr hilfreich
Eher nicht hilfreich Gar nicht hilfreich

4.3 An der UMG gibt es eine Verfahrensempfehlung zum Thema Patientenverfügung und Organspendeerklärung. Ist Ihnen diese Verfahrensempfehlung bekannt?

4.3.1 Ist Ihnen diese Verfahrensempfehlung bekannt?

Nein, ich habe von diesen Verfahrensempfehlungen noch nie gehört.

$\square$ Ja, ich habe von diesen Empfehlungen gehört. Ich habe sie aber nicht gelesen bzw. kann mich nicht mehr an deren Inhalt erinnern.

$\square$ Ja, der Inhalt dieser Empfehlungen ist mir bekannt.

4.3.2 Empfinden Sie diese Verfahrensempfehlung als hilfreich? (Bitte kreuzen Sie die zutreffende Antwort an)

Sehr hilfreich Hilfreich Eher nicht hilfreich Gar nicht hilfreich

\section{Persönliche Angaben}

5.1 Geschlecht:

weiblich

$\square$ männlich

5.2 Alter:

$\leq$ 30 Jahre $\square$ 30-39 Jahre $\square$ 40-49 Jahre $\square$ 50-59 Jahre $\square>60$ Jahre

5.3 Welcher Religionsgemeinschaft gehören Sie an?

keiner

römisch-katholisch

evangelisch

andere

Religion,

und

zwar: 
5.4 In welcher Klinik arbeiten Sie?

Klinik für Allgemein-, Viszeral- und Kinderchirurgie

Klinik für Anästhesiologie

Klinik für Gastroenterologie und Endokrinologie

Klinik für Kardiologie und Pneumologie

Klinik für Nephrologie und Rheumatologie

Klinik für Neurologie

Klinik für Pädiatrische Kardiologie und Intensivmedizin

Klinik für Unfallchirurgie

5.5 Welche berufliche Funktion nehmen Sie dort ein?

Arzt/ Ärztin

$\square$ Leiter/ Leiterin der Klinik

$\square$ leitender Oberarzt/ leitende Oberärztin

$\square$ Oberarzt/ Oberärztin

$\square$ Stationsarzt / Stationsärztin / Assistenzarzt/ Assistenzärztin

$\square$ PJ-ler/ PJ-lerin

Student/ Studentin

Gesundheits- und Krankenpfleger/ Krankenpflegerin

Stationsleitung

$\square$ Gesundheits- und Krankenpfleger/ Krankenpflegerin

Auszubildender/ Auszubildende

Sonstiges,

und

zwar:

\section{Vielen Dank für Ihre Mitarbeit!}




\section{$9 \quad$ Literaturverzeichnis}

aerzteblatt.de - Richtlinie der BÄK. DOI: 10.3238/arztebl.2015.rl_hirnfunktionsausfall_01; Zugriff am 17.04.2018

aerzteblatt.de - Zahl der Organspenden weiter rückläufig. https://www.aerzteblatt.de/nachrichten/77291/Zahl-der-Organspenden-weiterruecklaeufig; Zugriff am 13.03.2018

aerztezeitung.de - Weniger Organspenden: Das Problem Patientenverfügung. http://www.aerztezeitung.de/politik_gesellschaft/organspende/article/824317/wenigerorganspenden-problem-patientenverfuegung.html; Zugriff am 30.03.2015

BÄK (1993): Richtlinien der Bundesärztekammer für die ärztliche Sterbebegteilung. Dtsch Ärztebl 90, A2404-6

BÄK (1998): Grundsätze der BÄK zur ärztlichen Sterbebegleitung. Dtsch Ärztebl 92, A 2366-7

BÄK (2013a): Arbeitspapier zum Verhältnis von Patientenverfügung und Organspendeerklärung. Dtsch Ärztebl 110, A572-4

BÄK (2013b): Umgang mit Vorsorgevollmacht und Patientenverfügung in der ärztlichen Praxis. Dtsch Ärztebl 110, A 1580-5

Beauchamp TL, Childress JF: Principles of Biomedical Ethics. 5. Auflage; Oxford University Press, New York 2001

BGH Urteil vom 06.07.2016 Az. XII ZB 61/16: FamRZ 2016, 1671

BGH Urteil vom 13.09.1994 Az. 1 StR 357/94: BGH St 40, 257

BGH Urteil vom 17.03.2003 Az. XII ZB 2/03: BGHZ 154, 205

BGH Urteil vom 17.09.2014 Az. XII ZB 202/13,: NJW 2014, 3572 - Rn. 37; BVerfG, Urteil vom 20.12.2016, Az. 2 BvR 1541/15, NJW 2017, 1014 - Rn. 17)

Bobbert M: Patientenautonomie und Pflege: Begründung und Anwendung eines moralischen Rechts. Campus Verlag, Frankfurt am Main 2002

Breyer F, Daele W van den, Engelhard M (2007): Organmangel. Ist der Tod auf der Warteliste unvermeidbar? Dtsch Ärztebl 104, A-1086 / B-967 / C-919

bundesgesundheitsministerium.de - Gesetz zur Neuregelung der Organspende tritt in Kraft. http://www.bmg.bund.de/presse/pressemitteilungen/2012-03/neuregelung-zurorganspende.html; Zugriff am 03.08.2016

bundesgesundheitsministerium.de - Patientenrechte. http://www.bundesgesundheitsministerium.de/themen/praevention/patientenrechte/pati entenrechte.html; Zugriff am 15.08.2016 
Dehmel S (2006): Die Anfänge der Patientenverfügung in Deutschland. Hum Leben- Hum Sterb 4 , $50-52$

Deutsche Apotheker Zeitung (2012): Organspende und Patientenverfügung - geht das? AZ $\underline{48}, 7$

DGHS.de - Fakten zur Patientenverfügung. https://www.dghs.de/wissen/dghs-umfragen.html; Zugriff am 08.11.2016

DSO.de - Jahresbericht 2015: Organspende und Transplantation in Deutschland. https://www.dso.de/uploads/tx_dsodl/DSO_JB_2015_Web_2.pdf; Zugriff am 17.04.2018

DSO.de - Jahresbericht 2016: Organspende und Transplantation in Deutschland. https://www.organspende-info.de/sites/all/files/files/JB_2016_Web(1).pdf; Zugriff am 16.03.2018

DSO.de - Repräsentativbefragung: Einstellung, Wissen und Verhalten der deutschen Allgemeinbevölkerung zur Organ- und Gewebespende. http://www.dso.de/organspendeund-transplantation/thema-organspende/umfragen-zur-organspende.html; Zugriff am 11.08.2016

Duden.de - Autonomie: Rechtschreibung, Bedeutung, Definition, Synonyme, Herkunft. https://www.duden.de/rechtschreibung/Autonomie; Zugriff am 17.04.2018

Ethikrat.org - Die Zahl der Organspenden erhöhen. www.ethikrat.org/dateien/pdf/die-zahl-derorganspenden-erhoehen.pdf; Zugriff am 11.08.2016

Ethikrat.org - Hirntod und Entscheidung zur Organspende: Stellungnahme. http://www.ethikrat.org/publikationen/stellungnahmen/hirntod-und-entscheidung-zurorganspende; Zugriff am 16.04.2018

Evans N, Bausewein C, Meñaca A, Andrew EVW, Higginson IJ, Harding R, Pool R, Gysels M (2012): A critical review of advance directives in Germany: attitudes, use and healthcare

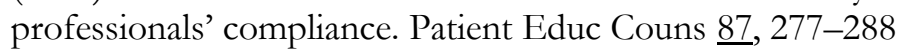

Faden RR, Beauchamp TL: A History and Theory of Informed Consent. Oxford University Press, New York 1986

Geisler LS (2004): Patient autonomy - a critical analysis. Dtsch Med Wochenschr 129, 453-456

Geißendörfer S: Die Selbstbestimmung des Entscheidungsunfähigen an den Grenzen des Rechts. (Ethik in der Praxis / Practical Ethics - Kontroversen / Controversies), Band 29; LIT Verlag, Berlin 2009

Grammenos D, Bein T, Briegel J, Eckardt K-U, Gerresheim G, Lang C, Nieß C, Zeman F, Breidenbach T (2014): Einstellung von potenziell am Organspendeprozess beteiligten Ärzten und Pflegekräften in Bayern zu Organspende und Transplantation. DMW - Dtsch Med Wochenschr 139, 1289-1294

Heberer J, Butzmann O, Eicher M, Hüttl PE: Organtransplantation, Patientenverfügung, Aufklärung und Einwilligung: Medizinrecht für Ärzte. J. Ecomed-Storck, Heidelberg 2013

Hickey M, Lewandowski L (1988): Critical care nurses' role with families: a descriptive study. Heart Lung J Crit Care 17, 670-676

Höfling W, Schäfer A: Leben und Sterben in Richterhand?: Ergebnisse einer bundesweiten Richterbefragung zu Patientenverfügung und Sterbehilfe. Mohr Siebeck Verlag, Tübingen 2006 
IfD-Allensbach.de - Deutlicher Anstieg bei Patientenverfügungen. http://www.ifdallensbach.de/uploads/tx_reportsndocs/PD_2014_20.pdf; Zugriff am 27.07.2016

Jox RJ (2012): Wie wird die Autonomie des Patienten am besten gewahrt? Letzte Entscheidungen bei Demenz und Wachkoma. Neurotransmitter 11, 44-47

Kant I: Grundlegung zur Metaphysik der Sitten. (Der philosophischen Bibliothek), Band 41, 3. Auflage; Felix Meiner Verlag, Hamburg 1965

Kant II: Beantwortung der Frage: Was ist Aufklärung? Kant ausgewählt und vorgestellt von Günter Schulte. Eugen Diederichs Verlag, München 1996

Klemperer D: Wie Ärzte und Patienten Entscheidungen treffen: Konzepte der Arzt-PatientKommunikation. Wissenschaftszentrum Berlin für Sozialforschung gGmbH, Berlin 2003

Klinkhammer G, Hibbeler B (2007): Organ- und Gewebetransplantation: Der menschliche Körper ist keine veräußerbare Ware. Dtsch Ärztebl 104, A-1458/ B-1296 / C-1236

Kuhlmann B (2004): Die Beziehung zwischen Angehörigen und Pflegenden auf Intensivstationen. Pflege 17, 145-154

Losch D, Hoffner M (2015): Organspende. Zbl Arbeitsmed Ergon 65, 272-274

Marckmann G, in der Schmitten J (2013): Patientenverfügungen und Advance Care Planning: Internationale Erfahrungen. Zbl Med Ethik 9ㅗ, 213-227

med.uni-goettingen.de - Wunsch nach Therapiebegrenzung und Bereitschaft zur Organspende. https://www.med.unigoettingen.de/de/media/V2_ethikkommitee/Verfahrensempfehlung_Patientenverfuegung _und_Organspendeerklaerung.pdf $+\& c d=1 \& h l=d e \& c t=c \ln k \& g l=$ de\&client $=$ firefox-b; Zugriff am 16.03.2018

Palandt O (Hrsg.): Bürgerliches Gesetzbuch. 77. Auflage; C.H. Beck, München 2018

Pilz N: Ist Lebendspende moralisch vertretbar? Studienarbeit Erfurt. GRIN-Verlag, München 2007

Reiter-Theil S (2005): Klinische Ethikkonsultation - eine methodische Orientierung zur ethischen Beratung am Krankenbett. Schweiz Ärzteztg 6ㅜ, 346-352

Reus K (2010): Die neue gesetzliche Regelung der Patientenverfügung und die Strafbarkeit des Arztes. JuristenZeitung $\underline{65}$, 80-84

Schäfer C (2012): Zukünftig mehr Organspender? Dial Aktuell 16, 315-315

Schell W: Staatsbürger- und Gesetzeskunde für Pflegeberufe in Frage und Antwort. 12. Auflage; Thieme Verlag, Stuttgart 2005

Störig HJ: Kleine Weltgeschichte der Philosophie. 16. Auflage; Kohlhammer, Stuttgart 1990

Uhlenbruck W (1978): Der Patientenbrief - die privatautonome Gestaltung des Rechtes auf einen menschenwürdigen Tod. NJW $\underline{31}$, S. 566-570

Ulsenheimer K, Biermann, Bock: Arztstrafrecht in der Praxis. (Praxis der Strafverteidigung), 5. Auflage; C.F. Müller, Heidelberg 2014

Verrel T (1999): Zivilrechtliche Vorsorge ist besser als strafrechtliche Kontrolle. Zum Stellenwert von Patientenverfügung, Betreuungsverfügung, Vorsorgevollmacht und vormundschaftsgerichtlicher Genehmigung. MedR - Med 17, 547-550 
Verrel T, Simon A: Patientenverfügungen: Rechtliche und ethische Aspekte. (Ethik in den Biowissenschaften / Sachstandberichte des DRZE 11); Karl Alber Verlag, Freiburg Br. 2010 


\section{Danksagung}

Mein besonderer Dank gilt meinem Doktorvater Herrn Professor Dr. phil. A. Simon der durch ausdauernde Geduld, kontinuierliche Beratung und konstruktive Kritik maßgeblich am Gelingen dieser Dissertation beteiligt ist.

Ebenfalls möchte ich mich bei den Mitarbeitern der Abteilung für Medizinische Statistik bedanken, die mich bei der statistischen Auswertung der Ergebnisse unterstützt und mich immer freundlich und gut beraten haben. 